\title{
Abstracts submitted to the Cell Therapy Transplant Canada 2020 Annual Conference
}

\author{
Cell Therapy Transplant Canada
}

Cell Therapy Transplant Canada thanks the following sponsors for providing educational grants to publish the CTTC 2020 abstracts in Current Oncology:

\section{Gold Level Educational Grant \\ Jazz Pharmaceuticals \\ (https://www.jazzpharma.com/)}

\section{Silver Level Educational Grant \\ Teva Canada}

(https://www.tevacanada.com/en/canada/)

\begin{abstract}
About Cell Therapy Transplant Canada
Cell Therapy Transplant Canada (СтTC) is an association of more than 400 clinicians, research scientists, cellular therapy laboratory technologists, pharmacists, nurses, patients, and caregivers across Canada. The сттC mission is to be the professional community and voice of hematopoietic stem cell transplant and cell therapy in Canada. The CTTC vision is to work together towards world-leading hematopoietic stem cell transplant and cell therapy for all Canadians.

The CTTC values excellence, innovation, integrity, collaboration, and professionalism in care, education, and research in hematopoietic stem cell transplant and cell therapy. The СтTC believes that every patient has a right of equal access to the highest quality of life-saving care that can be provided by hematopoietic stem cell transplant and cell therapy professionals in Canada.
\end{abstract}

To learn more about CTTC, please visit https://www.cttcanada.org/.

1. Implementing a frailty and functional assessment for patients undergoing allogeneic hematopoietic cell transplant during routine clinic visits

Ora Bascom, ${ }^{*}$ Leeann Wilson,* Maria Queralt Salas, ${ }^{*}$ Jonas Mattsson,* Shabbir M.H. Alibhai, ${ }^{\dagger}$ Rajat Kumar*

Background Frailty and poor functional status can adversely affect the outcome of patients undergoing allogeneic hematopoietic stem cell transplantation (alloнСт). Our alloHCT team felt the need to assess Fragility and Functional Status (F\&FS) of all alloHCT recipients as standard evaluation. This study summarizes the implementation of the program.

Methods In May 2018 the multidisciplinary transplant team members reviewed options for F\&FS assessment in our clinics with the aim of adopting Geriatric Assessment tools that were prognostic and easy to administer within the existing clinic appointment and space. After reviewing the literature, it was decided to adopt 8 different tests [details in Table 1 (https:// www.cttcanada.org/resource/resmgr/2020_conference/abstracts/1.PNG)] A one-page scoring tool was built by the clinical nurse coordinators to capture the results of each test [Figure 2 (https://www.cttcanada.org/ resource/resmgr/2020_conference/abstracts/2.PNG)]. If any part of the assessment was missed, it could be completed at the next visit or admission. Results It took the study team 45 days to implement the program. The tests are easy to perform and can be done by any clinical team member during the patient's transplant trajectory. There is no incremental increase in waiting time. Over a 6 -month period, 283 patients were assessed using the F\&FS. There was incomplete information in 57 of these patients, mainly during early implementation. The correlation of these test results with transplant outcomes was selected for presentation at the 2019 ASH meeting (abstract 380).

Discussion and Implications F\&FS assessment can be used as a standard of care testing for all patients being considered for alloHCT with proper planning and teamwork.

Affiliations: *Hans Messner Allogeneic Blood and Marrow Transplantation Program, Princess Margaret Cancer Centre, Toronto, $\mathrm{ON} ;{ }^{\dagger}$ Medicine, University Health Network, Toronto, ON.

2. Expanding the indications for ibrutinib: complete remission obtained by induction with ibrutinib followed by consolidative aHSCT in refractory primary CNS lymphoma

Natasha Pardy, Joanne Hickey

Introduction Primary central nervous system lymphoma (PCNSL) is an exceedingly rare and aggressive sub-type of non-Hodgkin lymphoma. Despite initial polychemotherapy that includes high-dose methotrexate, over half of patients will develop recurrent or refractory disease that requires salvage therapy. ${ }^{1}$ Ibrutinib, a Bruton tyrosine kinase inhibitor, has become an alternative for salvage treatment in relapsed or refractory PCNSL (RR-PCNSL) that is particularly useful in patients who are ineligible for re-induction with high-dose chemo. In RR-PCNSL, Ibrutinib led to a progression-free survival (PFS) of roughly five months when used as monotherapy $\mathrm{y}^{2,3}$ and 15 months when used as add-on therapy ${ }^{4}$ While its role as salvage treatment has been documented, its use to facilitate consolidative autologous hematopoietic stem cell transplant (aHSCT) in RR-PCNSL is not currently known. The following case describes the first known report of a patient with RR-PCNSL who achieved persistent complete remission following ibrutinib salvage treatment and consolidative aHSCT.

Case Description A 64-year-old male presented to the emergency department with a two-week history of ptosis, visual abnormalities, confusion, and increasing fatigue. On physical exam, he was found to have bilateral mydriasis, left third-nerve cranial palsy, severe left-sided ptosis, and restricted upwards and downward gaze of the right eye. Contrast computed tomography (СT) was performed that showed multiple areas of abnormal enhancement throughout the frontal lobes, corpus callosum, and midbrain associated with significant vasogenic edema. These findings were confirmed on magnetic resonance imaging (MRI). He underwent a stereotactic guided burr hole biopsy which was consistent with diffuse large B cell lymphoma (DLBCL). Immunohistochemistry performed on the tissue showed that the neoplastic cells were CD3(-), CD5(-), CD20(+), CD10(-), BCL2(subset +), BCL6(+), MUM1(+) and Cyclin D1(-). Staging Ст and bone marrow biopsy showed no evidence of systemic disease. He was diagnosed with PCNSL and went on to receive induction therapy with rituximab, methotrexate, procarbazine, and vincristine (R-MPV) with curative intent and received a total of 7 cycles. Initially, he had a significant radiographic response with a repeat MRI post-cycle 4 showing only a few small areas of residual enhancement. However, after completion of the 7 cycles of R-MPV, his MRI showed evidence of disease progression with both new and enlarging intra-axial lesions. Given his Eastern Cooperative Oncology Group performance status of 0 and lack of comorbidities, it was decided that he would proceed with salvage treatment with cytarabine and etoposide with curative intent for refractory PCNSL.

Unfortunately, after only 4 weeks of receiving cycle 1 of cytarabine and etoposide, a repeat MRI showed evidence of disease progression. He was then transitioned to palliative therapy with prednisone up until December 2017, at which point he was able to obtain ibrutinib on a compassionate

The opinions expressed in the abstracts are those of the authors and are not to be construed as the opinion of the publisher (Multimed Inc.) or the organizers of the CTTC 2020 annual conference. Although the publisher (Multimed Inc.) has made every effort to accurately reproduce the abstracts, Multimed Inc. and the organizers of the CTTC 2020 annual conference assume no responsibility and/or liability for any errors and/or omissions in any abstract as published. 
basis. He was started on ibrutinib salvage therapy and achieved radiographic evidence of complete remission after 4 months of treatment. There were minimal adverse effects of ibrutinib therapy, most notably a severe neutropenia requiring a temporary discontinuation of therapy for 2 weeks. He underwent consolidative aHSCT with thiotepa, busulfan and melphalan conditioning in August 2018. His post-transplant course was complicated by culture-negative febrile neutropenia with a subsequent source determined to be Clostridium difficile for which he was treated. A head MRI performed 3 months after his aHSCT showed no evidence of recurrent or residual disease. He continues to be followed by the Hematology Service in Newfoundland and has remained in complete remission since. Conclusions This case demonstrates the feasibility of a salvage approach using ibrutinib followed by aHSCT when standard salvage options have been exhausted in refractory PCNSL.

1. Grommes C, Nayak L, Tun HW, Batchelor TT. Introduction of novel agents in the treatment of primary cNs lymphoma. Neuro Oncol 2019;21:306-13.

2. Grommes C, Pastore A, Palaskas N, et al. Ibrutinib unmasks critical role of Bruton tyrosine kinase in primary CNS lymphoma. Cancer Discov 2017;7:1018-29.

3. Soussain C, Choquet S, Blonski M, et al. Ibrutinib monotherapy for relapse or refractory primary CNS lymphoma and primary vitreoretinal lymphoma: final analysis of the phase II "proof-of-concept" iLOC study by the Lymphoma study association (LYSA) and the French oculo-cerebral lymphoma (LOC) network. Eur J Cancer 2019;117:121-30.

4. Lionakis MS, Dunleavy K, Roschewski M, et al. Inhibition of B-cell receptor signalling by ibrutinib in primary cNS lymphoma. Cancer Cell 2017;31:833-43.e5.

Affiliations: Memorial University of Newfoundland, St. John's, NL.

\section{Outcomes of allogeneic transplant by disease site}

Dusyk Tanner,* Hahn Logan,* Sabry Waleed, ${ }^{\dagger}$ Elemary Mohamad, ${ }^{\dagger}$ Stakiw Julie, ${ }^{\dagger}$ Bosch Mark ${ }^{\dagger}$

Background Stem cell transplant (SCT) is a potentially curative option for patients with hematologic malignancies. An allogeneic SCT or the use of a donor's stem cells is associated with several clinically important outcomes. This study aimed to assess the outcomes and treatment efficacy associated with allogeneic SCT.

Purpose Currently, allogeneic SCT is treated similarly across varying types of hematologic malignancies. The present study identifies some disease-specific differences regarding overall survival (os), incidence of acute and chronic graft-versus-host disease (GvHD), and cause of death Methods A total of 166 allogenic SCT patients under the care of the Saskatchewan Bone Marrow Transplant Program between 2010 and 2019 were retrospectively reviewed. Each patient was evaluated for 4 main clinical outcomes: Os, incidence of acute and chronic GVHD, and cause of death. They were further divided into 5 classifications of hematologic malignancy (78 acute myeloid leukemia (AML), 16 acute lymphoblastic lymphoma (ALL), 20 chronic lymphocytic leukemia (CLL), 25 non-Hodgkin lymphoma (NHL), and 27 myelodysplastic syndrome (MDS). Data analysis was performed using the Power BI software application (Microsoft Corporation, Redmond, WA, U.S.A.) Results The best 10-year os was observed in CLL patients, at $60 \%$, while ALL had the worst 10 -year os at $40 \%$ ( $p=0.043$ ). The risk of acute GvHD was highest in CLL $60.0 \%$ and lowest in AML $32.5 \%$. Similarly, the incidence of chronic GVHD was highest in CLL $78.9 \%$ and lowest in AML $38.2 \%$. Relapse as a cause of death was highest for MDS (55.6\%) and lowest for NHL patients (20.0\%). The highest rate of infectious deaths was in ALL at $42.9 \%$.

Conclusions Clinical outcomes for allogeneic SCT are well known in the literature. However, the variability of outcomes based on disease is not well studied. This study shows that different diseases have predispositions to develop complications and outcomes based on the disease group. Since GvHD prophylaxis, disease monitoring, and infection prevention strategies are identical for all patients in the outpatient setting, the results of this study support a personalized approach to identifying disease complication based on hematologic malignancy.

Affiliations: *Department of Medicine, University of Saskatchewan, Saskatoon, SK; ${ }^{\dagger}$ Provincial Hematology and Blood and Marrow Transplant Program, Saskatchewan Cancer Agency, University of Saskatchewan, Saskatoon, SK.

\section{BeEAM conditioning regimen is an efficacious and economical} alternative to BEAM chemotherapy

Hahn Logan,* Dusyk Tanner,* Sabry Waleed, ${ }^{+}$Elemary Mohamad, ${ }^{+}$ Stakiw Julie, ${ }^{\dagger}$ Danyluk Pat, ${ }^{\dagger}$ Bosch Mark ${ }^{\dagger}$

Background Stem cell transplant (SCT) is an important therapeutic option for patients with malignant diseases such as lymphoma, leukemia, and multiple myeloma. The current standard of care for relapsed lymphoma consists of high-dose chemotherapy, followed by autologous SCT. Currently, the Saskatchewan Bone Marrow Transplant (SBMT) program utilizes a pre-transplant conditioning regimen consisting of bendamustine, etoposide, cytarabine and melphalan (BeEAM) for patients with relapsed or refractory lymphoma. Conditioning with BeEAM was introduced by the sBMT program in 2015 and replaced the BEAM protocol that utilizes carmustine (BCNU) instead of bendamustine.

Purpose Despite being the most frequently used conditioning regimen for 30 years, BEAM chemotherapy was associated with several adverse outcomes including interstitial pneumonitis. There is a paucity of information on the efficacy and safety of BeEAM chemotherapy with some sources citing BeEAM as an independent risk factor for non-relapse mortality and other sites reporting low rates of treatment-related mortality. Clearly, there is a need for the efficacy and safety of BeEAM conditioning to be assessed.

Methods Between 2010 and 2018, 131 lymphoma patients in Saskatchewan underwent an autologous SCT. This study compared the progression-free survival (PFS) and overall survival (OS) concerning the 2 conditioning regimens through a retrospective chart review. Additionally, a cost analysis was performed to elicit the savings associated with BeEAM therapy. The Power BI software application (Microsoft Corporation, Redmond, WA, U.S.A.) was used to generate Kaplan-Meier curves comparing survival in BEAM and BeEAM regimens.

Results Over an eight-year period, 88 patients were conditioned with BEAM and 43 were conditioned with BeEAM. There was no difference in os ( $p=$ $0.65)$ and PFS ( $p=0.63)$ between the treatment regimens. Economic analysis (list price) indicated that BEAM cost CA $\$ 33,381$ per transplant compared with CA $\$ 12,181$ for a BeEAM transplant, resulting in a net savings of roughly CA $\$ 890,000$ for the province throughout the study years.

Conclusions In this small study, BeEAm conditioning was equivalent to BEAM conditioning for autologous transplants in terms of os and PFS. Given the substantial cost savings associated with BeEAM, it will continue to remain the conditioning regimen of choice at our institution.

Affiliations: *Department of Medicine, University of Saskatchewan, Saskatoon SK; ${ }^{\dagger}$ Provincial Hematology and Blood and Marrow Transplant Program, Saskatchewan Cancer Agency, Saskatoon, SK.

5. Pre-transplant bone marrow cellularity and blood count recovery are not associated with relapse or survival risk following allogeneic stem cell transplant for AML in CR1

Igor Novitzky-Basso, ${ }^{+\dagger}$ Shiyi Chen, ${ }^{\dagger \ddagger}$ Jeffrey H. Lipton, ${ }^{* \dagger}$ Dennis D. Kim, ${ }^{*+}$ Auro Viswabandya, ${ }^{* \dagger}$ Rajat Kumar, ${ }^{+\dagger}$ Wilson Lam, ${ }^{* \dagger}$ Arjun Law, ${ }^{* \dagger}$ Zeyad Al-Shaibani, ${ }^{* \dagger}$ Armin Gerbitz, ${ }^{* \dagger}$ Ivan Pasic, ${ }^{* \dagger}$ Jonas Mattsson, ${ }^{*+}$ Fotios V. Michelis*t

Background Allogeneic hematopoietic cell transplantation (HCT) can be curative for acute myeloid leukemia (AML). Novel therapies may render patients' bone marrows hypocellular and lead to prolonged post-therapy pancytopenia.

Purpose Patients' bone marrow cellularity (BMC) at pre-transplant assessment (PTP) and post-treatment pancytopenia (leading to classification as CR-incomplete [CRi]) may be a manifestation of AML persistence, concerning for treating physicians.

Methods In this retrospective study, we examined the impact of BMC and post-treatment blood count recovery on a single-centre cohort of 342 patients who underwent HCT for AML in CR or CRi from January 2013 to December 2018. Data were updated November 2019. Median age was 57 years (range 18-73). Disease status at PTP was first complete remission (CR1) in $86 \%$ and CR $2+$ in $56 \%$ of patients. Myeloablative conditioning (MAC) was administered to $31 \%$ of patients while $69 \%$ received reduced-intensity conditioning (RIC). In vivo T cell depletion occurred in $75 \%$ of patients. Donors were matched-unrelated, matched-related and haplo-identical related $(200,108$, and 34$)$. Survivors' median follow-up was 31 months $(10-81)$. For BMC, patients were grouped as scoring aplastic $(<10 \%)$, hypocellular, normocellular and hypercellular for age $(22,89,174$, and 52 patients respectively; 5 patients had missing data); for ELN response (ELNr) at PTP all patients had BM blasts $<5 \%$ and those having neutrophils $\geq 1$ and platelets $\geq 100$ at PTP were deemed CR, otherwise CRi (190 and 152 patients respectively). As per Grimwade et al., 2010, MRC cytogenetic risk (MRC-R) was favourable in 21, intermediate in 227, adverse in 66, and unknown in 28. Cox proportional hazard model was used to analyze effects of variables on overall survival (OS) and Fine and Gray's competing risk regression model was used to examine effect of variables on cumulative incidence of relapse (CIR).

Results In total, os of the whole cohort at $2 y$ was $56.08 \%$, CIR was $20.31 \%$. CIR for BMC groups was aplastic $17.28 \%$, hypocellular $22.60 \%$, normocellular $19.63 \%$, hypercellular $21.89 \%, p$ nonsignificant (ns), [Figure 1 ( https:// cdn.ymaws.com/www.cttcanada.org/resource/resmgr/2020_conference/ 
abstracts/5_1.png)]; and for ELNr, CR $18.41 \%$, CRi $22.69 \%$, $p$ ns. The os for BMC was aplastic $63.64 \%$, hypocellular $49.39 \%$, normocellular $56.34 \%$, hypercellular $60.42 \%, p$ ns, and for ELNr, CR $56.97 \%$, CRi $55.06 \%, p$ ns. The distribution of MRC-R by BMC was not significantly different ( $p=0.3$ ) nor was primary induction failure ( $p=0.8)$, HCT-CI $(p=0.5)$ or usage of MAC compared with RIC $(p=0.9)$. The ELNr CRi was more likely in BMC aplastic and hypocellular, than CR $(p<2.6 \mathrm{e}-8)$. Multivariate analysis confirmed that neither BMC nor attainment of ELNr CR vs. CRi affected os or relapse. Other factors such as age at transplant $(p<0.0001)$ and chronic GvHD $(p=0.0003)$ influenced os, while MRC-R ( $p=0.0009)$ and chronic GvHD ( $p=0.001)$ affected relapse.

Conclusions Neither ELNr status (CR Vs. CRi) nor BMC at PTP influenced relapse post-HCT or Os. Hypocellularity and CRi should not be considered negative prognostic factors for post-HCT outcomes of AML.

Affiliations: *Hans Messner Allogeneic Transplant Program, Princess Margaret Cancer Centre, Toronto, ON; ${ }^{\dagger}$ University of Toronto, Toronto, ON; ${ }^{\ddagger}$ Department of Biostatistics, Princess Margaret Cancer Centre, Toronto, ON.

\section{Single UM171-expanded cord blood unit transplantation can cure severe aplastic anemia}

Jean-Sébastien Claveau, Sandra Cohen, Imran Ahmad, Jean-Sébastien Delisle, Thomas Kiss, Silvy Lachance, Guy Sauvageau, Lambert Busque, Rose-Marie Brito, Nadia Bambace, Léa Bernard, Denis Claude Roy, Jean Roy

Background Allogeneic hematopoietic stem cell transplantation (SCT) is currently the treatment of choice for young patients with severe aplastic anemia. In SCT candidates, the current priority order of donor is an HLA-identical sibling, an unrelated donor or an HLA-haploidentical donor (Georges GE, Blood Advances 2018). In patients missing a haplo-identical donor, cord blood ( $\mathrm{CB}$ ) transplantation offers a curative option. However, few adult cases of aplastic anemia transplanted with a single св have been reported due to insufficient number of progenitor cells in many $\mathrm{CB}$ grafts and fear of graft failure. The first-in-class pyridamole derivative which allows expansion of stem cells by an average factor of 35, UM171, could solve this caveat.

Case Description We describe the case of a 29 -year-old man with no past medical history diagnosed with severe aplastic anemia. He was heavily transfused and refractory to immunosuppressive therapy. A search for a suitable related or unrelated donor was unsuccessful. Absence of a single Св graft meeting our institutional criteria for Св SСТ in non-malignant diseases and encouraging clinical results using UM171-expanded CBs in high-risk hematologic malignancies (prompt engraftment, low mortality, rare chronic GvHD; (Cohen S, Lancet Haematology 2019) prompted us to perform the first UM171-expanded $\mathrm{CB}$ transplant in a patient with severe aplastic anemia.

Conditioning prior to transplant consisted of rabbit ATG $2 \mathrm{mg} / \mathrm{kg}$ (D-9 and D-8), fludarabine $30 \mathrm{mg} / \mathrm{m}^{2}$ (D-5 to D-2), cyclophosphamide $30 \mathrm{mg} / \mathrm{kg}$ (D-5 to $\mathrm{D}-2$ ) and TBI $2 \mathrm{~Gy}(\mathrm{D}-1)$. A GvHD prophylaxis consisted of MMF $1000 \mathrm{mg}$ TID from $\mathrm{D}+1$ to $\mathrm{D}+50$ (no taper) and tacrolimus from $\mathrm{D}-3$ to $\mathrm{D}+180$ and tapered completely 1 year post-SCT. Selected св for expansion was $6 / 8$ HLA-matched with $2.04 \times 10^{7} / \mathrm{kg}$ TNCs and $1.04 \times 10^{5} \mathrm{CD} 34+/ \mathrm{kg}$. СB was thawed and CD34+ cell enrichment was performed by immune-magnetic isolation (Miltenyi Canada) on D-7. Recovered CD34+ cells $\left(5.18 \times 10^{6}\right)$ were placed into culture media with UM171. Culture was injected daily with predetermined media volume and growth factors (IL-2, IL-6, stem cell factor, FLT-3 ligand). The patient received an expanded св graft of $3.29 \times 10^{6} \mathrm{CD} 34+$ cells $/ \mathrm{kg}$ (51-fold expansion) in a volume of $145 \mathrm{~mL}$ on D 0 . The CD34-component containing T lymphocytes $\left(2.58 \times 10^{6} \mathrm{CD} 3+/ \mathrm{kg}\right)$ was cryopreserved and infused on $\mathrm{D}+1$. Following infusion, we observed robust engraftment with $100 \%$ donor cells by $\mathrm{D}+14$. Day of monocytes $\geq 0.1 \times 10^{9} / \mathrm{L}$ was +8 , whereas days of absolute neutrophil count $\geq 0.1$ and $0.5 \times 10^{9} / \mathrm{L}$ were +10 and +23 respectively. Platelet engraftment was achieved on $\mathrm{D}+27$. The patient is currently +22 months with normal counts and no evidence of GVHD.

Discussion We report the first adult patient with aplastic anemia to benefit from an expanded CB transplantation without any major complication. Single UM171-expanded Св transplant offers a valuable alternative to adult patients with severe aplastic anemia without an available donor.

Affiliation: Division of Hematology, Oncology and Transplantation, Hôpital Maisonneuve-Rosemont, and Université de Montréal, Montréal, Québec, QC.

\section{The role of the allogeneic transplant nurse practitioner in the outpatient setting}

Peggy Chen, Zoe Evans,* Katherine Lee,* Kayla Madsen,* Gertrude Trinh*

In 2017, the allogeneic stem cell transplant (ASCT) program launched a new model of care for newly discharged allogeneic transplant patients. Historically, these patients were discharged to the outpatient allogeneic transplant clinic and care was provided by their transplant physician, and many patients were referred to the transfusion centre (TFC) for additional interventions. Under the present model, these patients are now discharged directly to TFC and managed by nurse practitioners (NPs) within a collaborative practice model. Patients typically remain in this setting until approximately two months after their ASCT and then transition to their physician follow-up clinics.

The primary mandate of this new model is to provide efficient and patient-centred care during the early phase of their post-transplant trajectory in the outpatient setting. This patient population requires frequent follow-up visits for assessment, diagnostic investigation, interventions, and care coordination. By providing these aspects of care in one setting, the number of health care providers previously utilized is reduced. The TFC provides NPs the flexibility to see patients outside the confinements of the physician clinic schedule, allowing for timely interventions and avoidance of emergency department (ED) visits.

Overall, the response to this model implementation has been positive. Continuity of care has improved and overall clinic times have decreased. This model allows the NPs to work to their full scope and promotes strong collaborative relationships within the interdisciplinary team.

Affiliation: *Princess Margaret Cancer Centre, Toronto, ON.

\section{Similar survival between myeloma patients relapsing after autologous or tandem autologous-allogeneic transplant} Jean-Sébastien Claveau, Richard LeBlanc, Imran Ahmad, Nadia Bambace, Léa Bernard, Sandra Cohen, Jean-Sébastien Delisle, Thomas Kiss, Silvy Lachance, Denis Claude Roy, Guy Sauvageau, Jean Roy

Background Compared with autologous (auto) hematopoietic stem cell transplantation (HSCT), better survival in patients with multiple myeloma (MM) progressing after tandem auto/allogeneic (allo) НSCT has been previously reported (Htut et al., BBMT 2018), suggesting a persistent graft-versus-multiple myeloma effect even after relapse. We sought to confirm this observation in 2 cohorts of MM patients transplanted in our institution. Methods We retrospectively analyzed 92 consecutive, newly diagnosed MM patients who received tandem auto/allo нSCT between 2001 and 2010 and paired them with 81 patients presenting similar characteristics who received auto HSCT only during the same period of time. These characteristics were age $\leq 65$ years at myeloma diagnosis, Durie-Salmon stages $1 \mathrm{~B}, 2$ or 3 , a glomerular filtration rate $\geq 60 \mathrm{~mL} / \mathrm{min}$ after first auto HSCT, use of only 1 line of induction treatment, absence of progression within 4 months of auto HSCT and absence of plasma cell leukemia at diagnosis. After their first auto HSCT with melphalan $200 \mathrm{mg} / \mathrm{m}^{2}$, all allo transplant recipients received an outpatient nonmyeloablative conditioning with fludarabine $30 \mathrm{mg} / \mathrm{m}^{2}$ and cyclophosphamide $300 \mathrm{mg} / \mathrm{m}^{2} \times 5$ days, followed by G-CSF-mobilized peripheral blood stem cells from a 6/6 matched sibling donor.

Results Median age in the auto/allo group was 53 years, compared with 57 years in the auto group $(p<0.001)$. Otherwise, both groups were similar for sex, immunoglobulin isotype, Durie-Salmon, and Iss stages. With a median follow-up of 13.1 and 10.2 years $(p<0.001)$ respectively, median overall survival (os) in the tandem group has not been reached, compared with 6.1 years in the auto group only [ $p<0.001$, Figure 1 (https:// www.cttcanada.org/resource/resmgr/2020_conference/abstracts/3. PNG)]. Overall survival and progression-free survival (PFS) at 10 years in tandem and auto groups were $61 \%$ compared with $37 \%(p<0.001)$ and $41 \%$ compared with $21 \%$ ( $p<0.001$ ) respectively. The 10 -year cumulative incidence of progression was significantly lower in the tandem group [49\% vs. $76 \%, p<0.001$, Figure 2(a) (https://www.cttcanada.org/resource/ resmgr/2020_conference/abstracts/4.PNG)]. Nonrelapse mortality (NRM) was higher in tandem recipients $(9 \%)$ without reaching statistical significance compared with the auto group $[2.5 \%, p=0.322$, Figure 2 (b) (https://www.cttcanada.org/resource/resmgr/2020_conference/ abstracts/5.PNG)]. Overall survival at 5 years and 10 years after first progression were $59 \%$ and $39 \%$ in the tandem group compared with $52 \%$ and $22 \%$ in the auto group respectively [ $p=0.06$ for both, Figure 3 (https:// www.cttcanada.org/resource/resmgr/2020_conference/abstracts/6. PNG)], with a similar second PFs. Following multivariate analysis of factors associated with progression, the only significant variable was tandem transplant (hazard ratio: 0.44 ; $95 \% \mathrm{CI}: 0.24$ to $0.80 ; p=0.008$ ), independently from age, Iss stage, type of induction and response before first auto HSCT. Main cause of death was refractory MM in both groups.

Conclusions Tandem auto/allotransplant enables long-term survival and offers a potentially curative option in selected newly diagnosed мм patients. At time of first relapse, outcomes of tandem auto/allo HSCT recipients and those who received auto HSCT only do not seem to differ significantly. Affiliation: Division of Hematology, Oncology and Transplantation, Hôpital Maisonneuve-Rosemont, and Université de Montréal, Montréal, Québec, QC. 


\section{Outcomes of adult patients with acute myeloid leukemia and inconclusive cytogenetics undergoing allogeneic hematopoietic stem cell transplantation}

Ram Vasudevan Nampoothiri, Shiyi Chen, Ivan Pasic, Zeyad Al-Shaibani, Wilson Lam, Fotios V. Michelis, Dennis (Dong Hwan) Kim, Auro Viswabandya, Armin Gerbitz, Jeffrey Howard Lipton, Rajat Kumar, Jonas Mattsson, Arjun Datt Law

Introduction Inconclusive cytogenetic analysis at baseline has been reported to be a poor prognostic feature in patients with acute myeloid leukemia (AML). We conducted this study to examine the prognostic impact of unsuccessful/inconclusive cytogenetic analysis in patients with AML on allogeneic hematopoietic stem cell transplantation (НSCT) outcomes. Methods We retrospectively analysed all adults ( $\geq 18$ years) undergoing HSCT for AML from January 2011 to August 2019. Patients with documented cytogenetic abnormalities by conventional karyotyping or fluorescence in situ hybridization were excluded. Inconclusive cytogenetics were defined as less than 20 dividing cells or metaphases seen during conventional karyotyping in either peripheral blood or marrow samples. Baseline characteristics and transplant outcomes were compared between patients with normal cytogenetics and those with unsuccessful/inconclusive cytogenetics. Kaplan Meier analysis was used for measuring outcomes.

Results Overall, 243 AML patients (median age 55 years, $55.1 \%$ women) were included. Inconclusive cytogenetics were reported in 79 patients while 164 patients had a normal karyotype. The 2 groups were similar to each other in baseline demographics, treatment received, and transplant-related variables like donor type (unrelated, sibling, or haploidentical), conditioning intensity myeloablative conditioning (MAC) vs. reduced-intensity conditioning (RIC), and type of graft-versus-host disease (GvHD) prophylaxis [Table 1 (https://www.cttcanada.org/resource/resmgr/2020_conference/ abstracts/8.PNG)]. There was no difference between patients with inconclusive cytogenetics and those with normal cytogenetics in terms of relapse-free survival (median 66 vs. 42 months, $p=0.53$ ) or overall survival [median 77 vs. 76 months, $p=0.72$, Figure 1(A,B) (https://www.cttcanada. org/resource/resmgr/2020_conference/abstracts/7.PNG)]. Survival parameters remained similar even in subgroup analysis according to MRC risk stratification based on NPM1 and FLT3 mutation status. In the subgroup of patients that were NPMlwt/FLT3wt, patients with inconclusive cytogenetics showed a trend towards worse overall survival (median 66 vs. 83 months, $p=0.27$ ). Univariate analysis for prognostic factors in patients of AML with inconclusive cytogenetics showed that age at $\mathrm{HSCT}<55$ years and presence of NPM1 mutation (in the absence of FLT3 mutation) were predictive of favourable outcomes. Only age at HSCT $<55$ years was predictive of favourable outcomes in multivariate analysis (hazard ratio $0.37, p=0.01$, Figure 1(C) (https://www.cttcanada.org/resource/resmgr/2020_conference/abstracts/7.PNG)].

Conclusions Outcomes after HSCT are comparable between AML patients with unsuccessful cytogenetic analysis and patients with normal cytogenetics even after stratification based on molecular risk factors. Allogeneic HSCT may mitigate the poor prognosis of unsuccessful cytogenetic analysis results in patients with AML. Incorporating next generation sequencing is likely to help further stratify patients where conventional karyotyping and molecular analysis does not provide sufficient prognostic information.

Affiliation: Hans Messner Allogeneic Blood and Marrow Transplant Program, Princess Margaret Cancer Centre, Toronto, ON.

\section{Enteral versus parenteral nutrition, what is best for transplant patients? \\ Kristen Brazel, Phillip Nguyen}

Patients who undergo a hematopoietic stem cell transplant are at high risk of developing gastrointestinal complications. This can range from mucositis, nausea and vomiting, malnutrition, graft-versus-host disease (GVHD), to neutropenic enterocolitis. It is our job to manage these complications and make sure that patients remain well-nourished throughout the transplant process. One of the ways to do that is to offer our patients either enteral or parenteral nutrition.

A systematic and literature review was conducted to evaluate the benefits and disadvantages of enteral and parenteral nutrition. At the moment, there are no clear guidelines to identify which might be better to minimize complications and therefore maximize recovery.

We are currently working under a provincial government where doing more with less is necessary to ensure care for all. This presentation will help nurses advocate for what is best for their patients.

Affiliation: The Ottawa Hospital, Ottawa, ON.

\section{Patient-reported health and quality of life following allogeneic stem cell transplantation}

Samantha Culos, ${ }^{*}$ Jennifer White, ${ }^{* \dagger}$ Stephanie Lee, ${ }^{\ddagger}$ Mary E. Flowers, ${ }^{\ddagger}$ Kevin Bray ${ }^{\ddagger}$

Background Patient-reported health and quality of life (QoL) are infrequently investigated among allogeneic hematopoietic stem cell transplant (allo-HSCT) recipients. However, these are arguably some of the most important outcomes from the patient standpoint and can be predictive of long-term survival. Furthermore, these outcomes may help identify priority areas for intervention.

Purpose The purpose of this research is to assess health and QoL from a patient's perspective following allo-HSCT.

Methods Recipients of allo-HSCT were emailed an online survey link on their transplant anniversary. This study was carried out in parallel with the methodology used at the Fred Hutchinson Cancer Research Center in Seattle, Washington. The survey included sections regarding activity level, overall health, and current medications. Survey responses were automatically stored in a Research Electronic Data Capture (REDCap) Web application.

Results Results are reported for year 1 of the survey (February 2018 to January 2019). There were 239 respondents and the average monthly response rate was $52 \%$. A majority of respondents $(79 \%, n=188)$ were $3+$ years post-transplant. Most respondents (67\%) reported their health to be good or very good. However, participants on immunosuppressive therapy for graft-versus-host disease (GvHD) had a significantly higher likelihood of reporting that their health was poor $(p<0.001)$. Of these, $32 \%$ of respondents reported being on steroids for GvHD at the time of the survey and $62 \%$ reported developing chronic GvHD at some point following transplantation.

Around half $(47 \%)$ of the respondents reported that their current health limited them a lot in vigorous activities, such as running or lifting heavy objects. However, a majority reported that their health did not limit them at all in less strenuous activities such as climbing one flight of stairs or walking one block. In all, $43 \%$ of respondents $(n=103)$ were in school or working either part-time or full-time, with $83 \%$ of these individuals being $3+$ years post-transplant.

In terms of late effects, $25 \%(n=57)$ reported taking medications for high cholesterol and $31 \%$ were taking medication for high blood pressure. Around $7 \%(n=17)$ of respondents reported being treated for a heart attack or angina, with a majority occurring post-transplant. Additionally, $40 \%$ $(n=93)$ of respondents reported developing cataracts, with $88 \%(n=82)$ of these individuals being $3+$ years post-transplant. Furthermore, 27\% ( $n=$ 61) reported receiving therapy for osteoporosis/osteopenia.

Conclusions Long-term follow up among allo-HSCT survivors revealed that a majority of individuals report being in good health. However, a significant proportion of patients remain unable to return to normal activities including work following transplant. The prevalence of post-transplant complications including GVHD and cardiovascular disease were comparable to other allo-HSCT populations. Not unexpectedly, patients with active GvHD were more likely to report poorer health.

Affiliations: *BC Cancer, Vancouver, BC; ${ }^{\dagger}$ Leukemia/Bone Marrow Transplant Program of BC, Vancouver, BC; ₹Fred Hutchinson Cancer Research Center, Seattle, WA.

\section{Effectiveness of exercise training to improve outcomes following hematopoietic cell transplantation: a systematic review of clinical studies.}

Joseph Aziz, ${ }^{*}$ Chloe Smith, ${ }^{+}$Mitchell Slobodian,* Risa Shorr,* Michael De Lisio, ${ }^{\dagger}$ David Allan*†

Background Hematopoietic cell transplantation (НСT) is an effective therapy used to treat patients with a variety of hematologic malignancies but is associated with toxicity, including the risk of infection, bleeding, and death associated with cytopenia before hematopoietic engraftment. Exercise can modify the bone marrow microenvironment and accelerate engraftment in mouse models of transplantation. A systematic review of clinical studies is needed to understand whether exercise is feasible, safe, and effective in patients undergoing нСт and to design definitive studies that use appropriate timing and intensity of exercise.

Methods In accordance with the PRISMA guidelines for systematic review reporting, a systematic search of the literature was performed using MEDLINE, PubMed, EMBASE, and the Cochrane Register of Controlled Trials. Studies of patients undergoing HCT were included and analysis focused on aspects of the exercise program and relevant clinical outcomes following transplant.

Results A total of 12 studies met the inclusion criteria for the systematic review (total of 512 enrolled participants; 263 enrolled in the study arms) with predominantly hematologic malignancies or bone marrow failure syndromes. Most studies enrolled adults only (10) and involved patients 
undergoing allogeneic (6), autologous (5), or either type of нСт. The primary source of cells were peripheral blood stem cells (5). Most exercise regimens featured low (4) or moderate intensity programs (4). Exercise modalities were variable but most commonly included stretching (6), resistance (5), and aerobic exercises (5). A total of 5 studies reported on engraftment which was not different between the exercise and control groups. Length of hospital stay was reported in 5 studies and was equivocal in both treatment and control groups. Graft-versus-host disease (GvHD) rates were reported in 5 studies 3 studies indicated a lower rate of GvHD with exercise, whereas 2 studies reported a higher rate of GvHD with the intervention; and 4 studies reported hemoglobin and platelet transfusion needs, which were also unchanged in the treatment arm when compared with controls. Survival was reported in 3 studies and was not changed by exercise.

Conclusions Exercise in patients receiving НСт is feasible and appears safe. Studies demonstrate significant heterogeneity regarding the intensity and specific type of exercise used and outcome reporting was inconsistent with only a minority of studies reporting transplant-related outcomes. Exercise of any intensity appears to have a modest impact on hematopoietic engraftment and other transplant-related clinical outcomes reported in the studies. Future studies with more standardized outcome measures will be needed to further clarify the impact of exercise on HCT.

Affiliations: *The Ottawa Hospital, Division of Hematology, and ${ }^{\dagger}$ University of Ottawa, Department of Kinesiology, Ottawa, ON.

\section{Preparation and characterization of cord blood growth factor-rich derivatives}

Marie-Eve Rheaume, ${ }^{*}$ Josee Perreault,* Diane Fournier, ${ }^{+}$Patrick Trépanier*

In recent years, there has been a significant decline in the demand for cord blood units (CBUs). This trend has led cord blood banks, including Héma-Québec's Public Cord Blood Bank, to explore other uses and processing methods for CBUs in order to exploit the full potential of this unique, valuable, and readily available product. Two main sources of biological product surplus have been identified. First, during the regular processing of CBUs, plasma and red blood cells, which are normally discarded, could be recovered. Second, units disqualified because of their low total volume or low cell counts, are also available. Here we describe a method that was used to obtain 4 derivatives from СBUs: plasma (СВР), growth factor-rich plasma (GFRP), forced serum and serum (CBS). The growth factor content of each of these products was characterized using a commercial Luminex kit allowing the measure of 14 growth factors. The results highlight the different anti-inflammatory growth factor content of the 4 CBU by-products, with CBS containing higher growth factor levels than GFRP and CBP for all but 1 growth factor. Our analysis also highlights some differences in the contents of cord-derived and adult plasma and serum. This work on the characterization of cord blood-derived products could lead to the development of substitutes to animal-based supplements used in tissue engineering or alternative preparation of therapeutic eye drops. These derivatives could be of interest given their low marginal cost of production alongside banking CBUs for hematopoietic stem cell transplants.

Affiliations: *Héma-Québec, Medical Affairs and Innovation, Québec, QC; ${ }^{\dagger}$ Héma-Québec, Stem Cells Laboratory and Cord Blood Bank, St-Laurent, QC.

\section{Full validation of a test for the rapid assessment of potency of cord blood hematopoietic stem cells using interleukin-3: the RAP-3 test}

Carl Simard*, Marie-Eve Rheaume*, Nellie Dumont*, Diane Fournier*

According to NetCord-FACT Standards, cord blood banks have to determine the potency of cord blood units (CBUs) on a representative sample of the cryopreserved product before release to a transplant centre. We recently developed a rapid assay based on the measurement of the physiologic response of CD34 cells to interleukin (IL) -3 stimulation by flow cytometry ${ }^{1}$. The IL-3 test was shown to be rapid $(24 \mathrm{~h})$, reproducible, specific, and sensitive for determining the potency and functionality of CBUs. In order to implement the test within our standard operating procedures, in a large collaborative effort between regulatory and quality specialists, our research department and stem cell laboratory performed a full method validation. Specificity, accuracy, inter- and intra-assay precision, as well as reproducibility, were determined using statistically significant sample groups of cryopreserved CBUs tested with the IL-3 protocol. We present our method and validation protocol, as well as part of our results and challenges. In summary, the validation has been completed without major quality deviations, and will allow for the integration of the technique within our regulated operations. We hope that sharing our process will inspire other laboratories to go forward with full method validation and adoption of this novel and rapid assay.
1. Simard C, Bonnaure G, Fournier D, Néron S. An objective flow cytometry method to rapidly determine cord blood potency in cryopreserved units. Transfusion 2019;59:2074-83.

Affiliations: *Héma-Québec, Québec, QC.

15. MSC-derived extracellular vesicles in preclinical animal models of cancer: systematic review and meta-analysis

Adrian Bailey,* Alvin Tieu, ${ }^{* \dagger}$ Manika Gupta, ${ }^{* \dagger}$ Mitchell Slobodian,*

Tim Ramsay, ${ }^{* \dagger}$ Rosendo Rodrigues, ${ }^{*}$ Manoj Lalu, ${ }^{* \dagger}$ David Allan*†

Background There is a growing body of literature that implicates mesenchymal stromal cell-derived extracellular vesicles (MSC-EVs) in the regulation of tumour growth. Studies remain preclinical and heterogeneous in their design. A systematic search and meta-analysis is needed to identify aspects of study design that appear most promising to determine the impact of MSC-EVs on cancer.

Methods and Results Through a systematic search of PubMed and Scopus, a total of 29 articles reporting on the effect of MSC-EVs on tumour growth were identified. Of those 29 studies, 19 reported outcomes related to tumour volume and weight. Based on evaluation of reporting using the SYRCLE tool, studies revealed a high degree of potential bias. Although substantial heterogeneity was observed between studies, there was a trend towards MSC-EVs inhibiting tumour growth, with an effect size of -1.36 (95\% confidence interval: -2.70 to -0.03 , with $I^{2}=90 \%$ ). An analysis of effectiveness was possible only when the heterogeneity between studies was sufficiently reduced by analyzing subgroups of studies based on specific aspects of study design or treatment variables. Manipulating MSC-EVs to express antitumour microRNAs, animal models that used tumour cell lines from the same species (that is, allogeneic non-human cancer cell lines), and studies of pancreatic cancer were associated with significant tumour regression following MSC-EV treatment compared with controls in our meta-analysis. Use of human cancer cells introduced marked heterogeneity in outcomes that precluded an assessment of efficacy, and the broad range of tumour tissue types that were tested was associated with marked heterogeneity in study outcomes. In many cases, only a single study for a particular tissue type was identified. Interestingly, MSCs cultured in hypoxic conditions prior to EV isolation and use of MSC-EVs in animal models of gastric cancer were associated with tumour progression.

Conclusions More studies are needed to improve the current foundation of preclinical evidence on MSC-EV tumour regulation. In order to accelerate research towards potential clinical trials, future preclinical studies should aim to reduce issues related to heterogeneity in study design as much as possible by considering the use of MSC-EVs that express antitumour microRNAss and/or focus on a particular cancer type, such as pancreatic cancer. Of note, the use of human cells in the animal model was associated with heterogeneity but retained more clinical relevance. Thus, performing more studies, particularly studies of each tissue type, will help in future assessments of efficacy of MSC-EVs on tumour regulation.

Affiliations: *Clinical Epidemiology and Regenerative Medicine, The Ottawa Hospital Research Institute, and †Faculty of Medicine, University of Ottawa, Ottawa, ON

16. Tolerance of cryopreserved peripheral blood stem cell freezing bags to shocks

Lucie Boyer,* Nathalie Dussault,* Patrick Trépanier, ${ }^{\dagger}$ Danny Brouard,*

Diane Fournier ${ }^{+}$

Purpose Héma-Québec offers cryopreservation services for peripheral blood stem cell (PBSC) storage and shipment to 4 Quebec hospital centres. In the last 5 years, we have experienced 3 occurrences of PBSC breakage out of more than 600 shipments. Although very rare, these events have major consequences for patients because of the uniqueness and invaluable characteristics of PBSCs. This project aimed to characterize the shock tolerance of cryopreserved PBSC bags and suggest different strategies to prevent breakage. Methods Cryopreservation bags of various volumes (Origen Biomedicals) were filled with maximum or average volumes of RPMI- $10 \%$ dimethyl sulfoxide (DMSO) freezing medium or $0.9 \%$ saline solution and inserted into metal cassettes of appropriate sizes following current procedures at the stem cell laboratory of Héma-Québec. Filled bags were frozen and transferred to a nitrogen vapour tank. The internal temperature of larger bags during the freezing process was monitored. Adrop test has been specifically developed to characterize the shock amplitude absorbed by the cassettes using a G-Force data logger (VB300, Extech Instruments) when subjected to repeated drops of $h=50 \mathrm{~cm}$. The efficiency of various shock mitigation strategies was also investigated.

Results The temperature profiles of cryopreserved PBSC bags filled with either saline or RPMI- $10 \%$ DMSO were significantly different during the 
freezing process. The saline model demonstrated higher breakage event rates compared with RPMI-10\% DMSO under the same testing conditions. Using maximum saline volumes (CS1000), the breakage event rate was $38 \%$, declining to $23 \%$ for CS750, $7 \%$ for CS500, and $0 \%$ for CS250, suggesting a relationship between the observed breakage event rates and bag weight The use of sponge gauze led to a significant decrease in breakage event rates. An overall decline in breakage rates was observed for bags filled with RPMI-10\% DMSO, for which $100 \%$ (17/17) of sterility breaches occurred at the bottom of the bags, whereas $63 \%(22 / 35)$ of saline bags showed complete bursting. A breakage rate of $\sim 10 \%$ was found for larger bags (CS1000 and CS750) that were filled to the maximum with RPMI-10\% DMSO, with a high variability regarding the shock resistance, as some broke after only one $50 \mathrm{~cm}$ drop, while others remained intact after up to 10 drops. There was no direct correlation between the measured drop acceleration ( $G$ force) and the observed breakage rate.

Conclusions The use of RPMI-10\% DMSO is believed to represent a more reliable model to assess breakage event rates during PBSC processing and transport. Considering that PBSC bag breakages are rare, our study demonstrates that shock amplitude or frequency (or both) must be significantly high to lead to a sterility break. Our results suggest that the reduction of component volumes might reduce breakage rates.

Affiliations: *Héma-Québec, Medical Affairs and Innovation, Québec, QC; ${ }^{\dagger}$ Héma-Québec, Stem Cell Laboratory, Montréal, QC.

\section{Earlier steroid use with axicabtagene ciloleucel (axi-cel)} in patients with relapsed/refractory large $B$ cell lymphoma Max S. Topp, ${ }^{*}$ Tom van Meerten, ${ }^{\dagger \neq}$ Roch Houot, $\$$ Monique C. Minnema, Noel Milpied, ${ }^{*}$ Pieternella J. Lugtenburg, ${ }^{\neq * *}$ Catherine Thieblemont, ${ }^{+\dagger}$ Martin Wermke, ${ }^{\neq \neq}$Kevin W. Song, ${ }^{\S}$ Irit Avivi, III John Kuruvilla, \#\# Ulrich Dührsen, ${ }^{* * *}$ Rachel Chu, ${ }^{+++}$Lianqing Zheng, ${ }^{+++}$Vicki Plaks, ${ }^{+++}$ Anne Kerber, ${ }^{+++}$Marie José Kersten ${ }^{\ddagger \neq \neq}$

Background Axi-cel is an autologous anti-CD19 chimeric antigen receptor (CAR) T cell therapy approved for the treatment (Tx) of patients (pts) with relapsed/refractory large B cell lymphoma (R/R LBCL) with $\geq 2$ prior systemic therapies. In cohorts $1+2(\mathrm{C} 1+2)$ of ZUMA- 1 , the multicentre, single-arm registrational phase $1 / 2$ study of axi-cel in pts with refractory LBCL, grade 3 or greater cytokine release syndrome (CRS) and neurologic events (NEs) occurred in $11 \%$ and $32 \%$ of pts respectively (Locke et al., Lancet Oncol 2019). A safety expansion cohort was added [cohort 4 (C4)] to evaluate the effect of earlier steroid use on the rates of CRS and NEs. Here we present the primary analysis of ZUMA-1 C4.

Methods Eligible pts were leucapheresed and could receive optional bridging chemotherapy before conditioning chemotherapy and axi-cel infusion at a target dose of $2 \times 10^{6}$ anti-CD19 CAR T cells $/ \mathrm{kg}$. Patients in C4 received early steroid intervention starting at grade 1 NEs and at grade 1 CRS when no improvement was observed after 3 days of supportive care. The primary endpoints were incidence and severity of CRS and NEs. Additional endpoints were efficacy outcomes and biomarker analyses.

Results As of 6 May 2019, 41 pts had received axi-cel, with a median F/U of 8.7 mo. Patients who received bridging therapy prior to axi-cel $(68 \%)$ all had evidence of disease after bridging. The median age was 61 years (range, $19-77 ; 32 \% \geq 65$ ). Nearly half of all pts (49\%) had an Eastern Cooperative Oncology Group (ECOG) performance status of $1,70 \%$ had disease stage III/IV, $68 \%$ were refractory to $\geq 2$ nd-line therapy, $12 \%$ were relapsed to $\geq 2$ nd-line therapy, $63 \%$ had $\geq 3$ prior lines of therapy, and $20 \%$ had relapsed after autologous stem cell transplantation (ASCT). Overall, pts enrolled in $\mathrm{C} 4$ had a lower median tumour burden by sum of product diameters [SPD (C4: $\left.2100 \mathrm{~mm}^{2} ; \mathrm{C} 1+2: 3723 \mathrm{~mm}^{2}\right)$ ] compared with $\mathrm{C} 1+2$.

As expected from the revised adverse event (AE) management, a greater proportion of pts in $\mathrm{C} 4$ received steroids and tocilizumab vs. $\mathrm{C} 1+2(73 \%$ and $76 \%$ vs. $27 \%$ and $43 \%$ ). Fewer pts in C 4 experienced grade $\geq 3$ CRS ( $2 \%)$ and NEs (17\%) than was previously observed in $\mathrm{C} 1+2$. The overall response rate (ORR) in $\mathrm{C} 4$ was $73 \%$ with a complete response (CR) rate of $51 \%$. Fifty-four percent of pts remained in ongoing response with $\geq 6 \mathrm{mo} \mathrm{F} / \mathrm{U}$, compared with the $44 \%$ ongoing response rate at the primary analysis of $\mathrm{C} 1+2$ (also $\geq 6 \mathrm{mo} \mathrm{F} / \mathrm{U}$ ). While pts in $\mathrm{C} 4$ generally had lower SPD than those in $\mathrm{C} 1+2$, the responses were comparable between cohorts when evaluated by tumour burden. Median progress-free survival (PFS) was $11.7 \mathrm{mo}$, median overall survival (OS) was not reached; CAR T cell expansion was comparable between $\mathrm{C} 1+2$ and 4 . Conclusions Earlier steroid use appears to reduce the rate of CAR T cell Tx-related CRS and NEs in C4 compared with $\mathrm{C} 1+2$, without a clinically meaningful impact on efficacy. Conclusions are limited by the nonrandomized study design, differences in population sizes, and baseline characteristics between cohorts, as well as the potential confounding effect of bridging therapy. Optimizing AE management is important to improve the benefitrisk profile of CAR T cell therapy.
Affiliations: *Medizinische Klinik und Poliklinik II, Universitätsklinikum Würzburg, Würzburg, Germany; †University Medical Center Groningen, Groningen, Netherlands; ${ }^{\ddagger}$ On behalf of HOVON/LLPC (Lunenburg Lymphoma Phase I/II Consortium); ${ }^{\circledR}$ University Hospital of Rennes, Rennes, France; |lUniversity Medical Center Utrecht, Utrecht, Netherlands; ${ }^{\#} \mathrm{CHU}$ Bordeaux, Service d'hematologie et therapie Cellulaire, F-33000, Bordeaux, France; **Erasmus MC, HOVON/LLPC (Lunenburg Lymphoma Phase I/II Consortium), Rotterdam, Netherlands; ${ }^{\dagger \dagger}$ Hôpital Saint Louis, Paris, France; ${ }^{\ddagger \ddagger}$ NCT/UCC Early Clinical Trial Unit, University Hospital Carl Gustav Carus, Dresden, Germany; ${ }^{\S}$ University of British Columbia, Vancouver, BC; "IIIISourasky Medical Center, Tel-Aviv, Israel; "\#University of Toronto, Toronto, ON; ${ }^{* * *}$ University Hospital Essen, Essen, Germany; ${ }^{\dagger \dagger}$ Kite, A Gilead Company, Santa Monica, CA, U.S.A.; ${ }^{\ddagger \ddagger}$ Academic Medical Center, University of Amsterdam, Amsterdam, Netherlands.

\section{Is there any impact of uric acid levels during the peri- transplant period on acute graft-versus-disease incidence and allotransplant outcome?}

Panayotis Kaloyannidis,* Eshrak Shaibani,* Hussin Alsubaie,* Rawan Omari,* Solaf Kanfar,* Zahra Al Shammasi,* Kenana Ajlan, ${ }^{+}$Norah Mohlam, ${ }^{\dagger}$ Abdulhamid Liacini, ${ }^{\dagger}$ Ahmed Bahrani, ${ }^{\ddagger}$ Hani Al Hashmi,* Khalid Al Anezi*

Background Uric Acid (UA) belongs to the damage-associated molecular patterns (DAMPs), which have recently been considered important contributors to the acute graft-versus-host disease (aGvHD) pathogenesis. Currently, only two published studies evaluated the role of UA as aGvHD mediator, with conflicting results. Given that aGvHD is a dynamic process, in contrast to previous studies, we evaluated serum UA levels not only once but in different time points during the early post-transplant period and investigated its correlation to aGvHD incidence, non-relapse mortality (NRM), and survival rates.

Methods We retrospectively evaluated 57 patients (pts), with a median age of 36.8 years (range, 17-62), allografted from full matched sibling donors for malignant $(n=48)$ or nonmalignant $(n=9)$ hematologic diseases. A median of $5.6 \times 10^{6} / \mathrm{kg}$ CD $34+$ cells were infused after a myeloablative $(n=$ $34)$ or reduced intensity $(n=23)$ regimen. At the time of allogeneic stem cell transplant (allosCT), 42 pts were in remission (CR1:30; CR2: 10; >CR2: 2). All pts received either allopurinol or rasburicase from the day of conditioning initiation to day -1 . The Roc-curve method, $t$-test, Kaplan-Meier method, and log-rank test were used for the univariate analysis while the binary logistic regression method was used for the multivariate statistical analysis. Results The median UA levels were $3.2,2.4,2.2$, and $2.9 \mathrm{mg} / \mathrm{dL}$ at days -7 , $0,+7$, and +14 respectively; using the Roc curve method the cutoff point was determined at $4.4 \mathrm{mg} / \mathrm{dL}$ for day -7 and $2.2 \mathrm{mg} / \mathrm{dL}$ for days $0,+7$, and +14 . Overall, 20/57 (35\%) pts developed aGvHD; 18 (31\%) were assessed as grade $\geq$ II, while $10(17 \%)$ as grade III-IV. The incidence of the aGvHD grade $\geq$ II was higher for pts with UA levels $\geq 2.2 \mathrm{mg} / \mathrm{dL}$ at days 0 (12 vs. $6 \mathrm{pts}$ ) and +14 (13 vs. 5 pts). However, these differences did not reach statistical significance. Uric acid levels at +7 day had no impact on aGvHD incidence. In multivariate analysis, only the number of CD34 $+>6 \times 10^{6} / \mathrm{kg}$ adversely affected the aGvHD grade $\geq$ II $(p=0.04)$ while intermediate and advanced disease phase along with a number of CD34+ cells $>6 \times 10^{6} / \mathrm{kg}$, proved to be significant contributors for severe aGvHD (gr III-IV) occurrence $(p<0.03)$. A better 4-year overall survival was noticed for pts with UA levels $<2.2 \mathrm{mg} / \mathrm{dL}$ at day $+14(85 \%$ vs. $55 \%, p=0.06)$. Fifteen pts succumbed to NRM causes; $8 / 15$ deaths were related to aGvHD. The NRM was higher in patients who had UA levels $\geq 2.2 \mathrm{mg} / \mathrm{dL}$ at day $+14(32 \%$ vs. $15 \% p=0.2)$.

Conclusions The present study bears the limitations of a small series of pts and is retrospective nature. However, it has the advantage of evaluating UA levels as an aGvHD mediator, at multiple time points during the peri-transplant period. Our results, though not of strong statistical significance, demonstrated that UA levels might affect aGvHD incidence as well as survival and the NRM rates post-allosCT. Well-designed prospective clinical trials are definitely warranted to clarify the role of UA on allosCT outcome.

Affiliations: *Adult Hematology and Stem Cell Transplantation Department, ${ }^{\dagger}$ Laboratory Technologist Department, and ${ }^{\ddagger}$ Pathology and Lab Medicine Department, King Fahad Specialist Hospital, Dammam, Saudi Arabia.

\section{Defibrotide for the treatment of hepatic veno-occlusive disease/sinusoidal obstruction syndrome after hematopoietic cell transplantation or chemotherapy: interim results from the DEFIFrance study}

Mohamad Mohty, ${ }^{*}$ Myriam Labopin, ${ }^{*}$ Ana Berceanu, ${ }^{+}$Charlotte Jubert, ${ }^{\ddagger}$ Stéphane Girault, $\$$ Marie Detrait, "l Cécile Pochon, ${ }^{\sharp}$ Virginie Gandemer,*** Régis Peffault de Latour, ${ }^{+\dagger}$ David Michonneau, ${ }^{+\dagger}$ Floriane Delaval, ${ }^{\neq \neq}$ Anne Sirvent, $\$ \S$ Laurence Clément, IIII Anne Huynh, \#\# Maya Isaila,*** Virginie Bouvatier, ${ }^{++\dagger}$ Raj Hanvesakul, ${ }^{\neq \neq \neq}$Jean-Hugues Dalle ${ }^{\S \S}$ 
Background Hepatic veno-occlusive disease/sinusoidal obstruction syndrome (VoD/sos) is a potentially fatal complication of conditioning for hematopoietic cell transplantation (НСT) that may also occur after chemotherapy alone. With supportive care alone, voD/sos with multiorgan failure (MOF) is associated with $>80 \%$ mortality. Diagnosis of vOD/sOs traditionally used Baltimore or modified Seattle criteria, but in 2016 the European Society for Blood and Marrow Transplantation (ЕВMT) published separate diagnostic criteria for adult and pediatric patients (pts). Defibrotide is approved in Canada and the European Union (EU) for treating severe hepatic VOD/sos post-HCT in adult and pediatric pts (aged $>1$ month in the EU) and for hepatic VOD/sos with renal/pulmonary dysfunction post-HCT in adult and pediatric pts in the United States.

Purpose To collect real-world data on the safety and efficacy of defibrotide in French pts. Interim analysis results are presented.

Methods DEFIFrance is an observational, post-marketing study collecting retrospective and prospective data on pts receiving defibrotide in France since July 2014. This interim analysis is based on a November 2018 data cut-off and includes pts with severe and very severe vOD/sos post-HCT or post-chemotherapy from 36 HCT centres. vOD/sos was diagnosed using traditional criteria and severity was categorized using the EBMT criteria (adults) or study steering committee member adjudication (pediatric pts). Kaplan-Meier-estimated day 100 survival and day 100 complete response (CR; total serum bilirubin $<2 \mathrm{mg} / \mathrm{dL}$ and MOF resolution per investigators' assessment) and safety are reported.

Results Of the 303 pts (177 adults; 126 pediatric) with a documented reason for receiving defibrotide, 140 were diagnosed with voD/sos (99 adults, 41 pediatric; 120 diagnosed post-HCT, 20 diagnosed post-chemotherapy). Of these, 105 pts ( 74 adults; 31 pediatric) had severe or very severe vOD/ sos: 49 severe VOD/sos ( 31 adults, 18 pediatric) and 56 very severe vOD/sos (43 adults, 13 pediatric).

Overall, day 100 Kaplan-Meier-estimated survival was 58\% (95\% CI: $49 \%-66 \%$ ) for all pts with voD/sos [Figure 1 (https://www.cttcanada.org/ resource/resmgr/2020_conference/abstracts/9.PNG)]. The estimated day 100 survival rate was 55\% (95\% CI: $45 \%-64 \%)$ in the combined severe/ very severe vOD/sos subgroup [severe: $79 \%$ (95\% CI: $64 \%-88 \%)$; very severe: $34 \%$ (95\% CI: $21 \%-47 \%$ )]. Day 100 CR rates were $53 \%$ in the overall group and $52 \%$ in the combined severe/very severe voD/sos subgroup (severe: 77\%; very severe: $28 \%$ ) [Figure 2 (https://www.cttcanada.org/resource/ resmgr/2020_conference/abstracts/10.PNG)]. The table at https://www cttcanada.org/resource/resmgr/2020_conference/abstracts/11.PNG summarizes AEs of interest.

Conclusions This interim analysis of the DEFIFrance registry demonstrated an estimated day 100 survival of $58 \%$ in pts with voD/sos and $55 \%$ in pts with severe/very severe vOD/sos (per EBMT grading criteria in adults and expert adjudication in pediatric pts). The higher estimated survival for severe $(79 \%)$ versus very severe (34\%) vOD/sos highlights the importance of $\mathrm{vOD} / \mathrm{sos}$ diagnosis and treatment before pts reach the most severe voD/sos stages. Survival, CR rate, and safety findings, consistent with prior clinical trials, provide supportive evidence for the clinical utility of defibrotide for treatment of vOD/sos.

Affiliations: *Hôpital Saint-Antoine, Paris, France; †Hôpital Jean Minjoz Besançon, France; ${ }^{\ddagger}$ Hôpital des Enfants, Bordeaux, France; ${ }^{\S}$ Hôpital Dupuytren-CHU Limoges, Limoges, France; $\|_{\mathrm{CHU}}$ de Nancy, Nancy, France; "Hôpital d'Enfants de Brabois, Nancy, France; ${ }^{* *} \mathrm{CHU}$ de Rennes, Rennes, France; ${ }^{\dagger \dagger}$ Hôpital Saint-Louis, Paris, France; ${ }^{\dagger \dagger} J a z z$ Pharmaceuticals, Paris, France; ${ }^{\ddagger \ddagger}$ Hôpital Arnaud de Villeneuve, Montpellier, France; ${ }^{\S}$ Hôpital Haut-Lévêque, Pessac, France; IIIIInstitut Universitaire du Cancer de Toulouse-Oncopole, Toulouse, France; \#\#azz Pharmaceuticals, Oakville, ON; ***Jazz Pharmaceuticals, Lyon, France; ${ }^{\dagger \dagger}$ Jazz Pharmaceuticals, Oxford, U.K.; ${ }^{\S \S}$ Hôpital Robert-Debré, GHU Paris-Nord Université de Paris, France.

\section{Pre-processing evaluation of fresh cord blood stem and progenitor cells' potency using a modified RAP-3 test}

Guillaume Margaillana, Pascal Rouleaua, Nellie Dumonta, Mathieu

Drouina, Josée Laganièrea

Beyond the requirement for compatibility between donor and patient, cord blood banks select cord blood units (CBUs) for transplant on the basis of quality criteria defined by regulatory agencies and according to cord blood standards such as those of FACT and AABB. One of these criteria is the determination of cell regeneration capacity, called "potency measurement" of cryopreserved units prior to distribution to the transplant centre. Currently, potency is measured using the colony-forming unit (CFU) assay, in which cells are cultured in methylcellulose medium containing a combination of growth factors including interleukin-3 (IL-3). The long incubation time required for this test (7-14 days before final results are available), as well as the manual CFU counting, entails major reproducibility, robustness, time, and cost drawbacks. Recently, Héma-Québec has developed a flow cytometry test to rapidly assess the potency of cord blood units (CBUs) based on the detection of STAT5 activation in CD34+ cells in response to IL-3 stimulation named RAP-3 (Rapid Assessment of Potency with IL-3). The results of this test correlate well with CFU counts ${ }^{1}$, and the value of this test will be further assessed in an upcoming multicentre study.

Although the RAP-3 assay was first intended to evaluate CBU potency post-thaw, and was thus optimized for cryopreserved cord blood, the assay could also be used for the rapid assessment of the quality of a cord blood, as well as of different pre-processing treatments. In addition, analysis of different variables, such as the impact of the time interval before processing, of anticoagulant solutions, of the shaking method, or of storage temperature before volume reduction, could potentially help further improve clinical grade CBU quality. These considerations prompted us to optimize the event count and the signal-to-noise ratio of the RAP-3 assay for direct evaluation of potency in fresh cord blood. Results on the impact of different treatments on the CD34+ cell potency using this optimized test will be presented.

In summary, the application of the RAP-3 test to fresh cord blood offers an opportunity to rapidly evaluate critical parameters that might enhance the quality of CBUs.

1. Simard C, Bonnaure G, Fournier D, Néron S. An objective flow cytometry method to rapidly determine cord blood potency in cryopreserved units. Transfusion 2019;59:2074-83.

Affiliation: Héma-Québec, Québec, QC.

\section{Cellular therapy using TH9402 increases functionally competent Treg population in patients with chronic GvHD} Jean-Philippe Bastien,* Vibhuti Dave, Sandra Cohen, David Allan, ${ }^{\dagger}$ Donna Hogge, ${ }^{\ddagger}$ Denis-Claude Roy ${ }^{\S}$

Even the most potent immunosuppressive drugs often fail to control graft-versus-host disease (GVHD), the most frequent and deleterious post-transplantation adverse condition. We previously reported that photodepletion (PD) using dibromorhodamine (TH9402) eliminates T cells from healthy donors activated against major histocompatibility complex (MHC)-incompatible cells and spares resting T cells. In the present study, we identified PD conditions selectively eradicating $60 \%-90 \%$ of endogenous host-reactive $\mathrm{T}$ cells from chronic GvHD patients, with concomitant sparing of more than $50 \%$ of CD4+CD25+FoxP3+ regulatory T cells (Tregs). Moreover, these spared Tregs display an inhibitory effect on cGvHD cell proliferation. This effect is attributable to cellular contact between PD cells and cGVHD cells and requires CTLA-4 ligation. Furthermore, we observed that the co-culture of cGvHD cells with PD cells was associated with an increase in the expression of indoleamine 2,3 dioxygenase (IDO). This induction of IDO was dependant on CTLA-4 ligation. Interestingly, the inhibition of IDO resulted in hampered anti-proliferative activity by PD cells with concomitant increase in interferon gamma and decrease of IL-10 expression in CD4+ T cells. We also observed that co-culture of PD cells with cGvHD cells was capable of inducing Treg phenotype in CD4+CD25- cells through IDO-dependent mechanism. These anti-proliferating and Treg-generating properties of IDO could be used in vivo for the generation of Tregs in patients with cGvHD. Hence, we found that patients treated with TH9402-based cellular therapy displayed increases in FOXP3+ Treg population. In conclusion, these results identify a novel approach to selectively eliminate host-reactive T cells while sparing and expanding Tregs. These results could fill the need for novel therapeutic strategies for immunosuppressive refractory cGvHD.

Affiliations: *Maisonneuve-Rosemont Hospital Research Centre, Montréal, QC; ${ }^{\dagger}$ University of Ottawa, Ottawa, ON; ${ }^{+}$University of British Columbia, Vancouver, $\mathrm{BC}$; ${ }^{\S}$ Université de Montréal, Montréal, QC.

\section{Maturation of ex vivo-cultured human erythrocytes and sickle cell disease modelling using CRISPR-Cas9}

Yelena Boccacci, ${ }^{* \dagger}$ Guillaume Margaillan, ${ }^{* \dagger}$ Nellie Dumont, ${ }^{*}$ Mathieu Drouin, ${ }^{*}$ Yannick Doyon, ${ }^{\dagger \neq}$ Josée Laganière*t

Red blood cells (RBCs) are widely used in transfusion medicine, but the supply is entirely dependent on volunteer donors. The production of RBCs in vitro has been proposed as an alternative source to address the anticipated blood shortages in the coming years due to the aging population. In addition, in vitro-produced RBCs could be used as cellular models for studying erythroid diseases or for providing reagent cells expressing rare blood group antigens useful for immunohematologylaboratories. However, the non-fully mature state of cells achieved with current erythroid differentiation protocols is a significant barrier to the in vitro production of RBCs.

We aimed at modifying existing differentiation protocols in order to improve final erythroid maturation from human hematopoietic stem and progenitor cells (HSPCS). If successful, the method would facilitate 
preservation of cultured erythrocytes with commonly used red cell preservation solutions. In addition, the generation of mature erythrocytes would allow phenotype evaluation. As a proof-of-concept using gene editing we chose to model the "sickling" phenotype typical of sickle cell disease.

After significant improvements of currently known methods, we devised an optimized culture medium for the efficient erythroid differentiation and maturation of adult peripheral blood HSPCs. Using this procedure the erythroid differentiation protocol yielded up to $95 \%$ of enucleation by day 22. More importantly, at day 35, reticulocyte maturation yielded up to $80 \%$ of cells molecularly defined as mature erythrocytes, being devoid of any CD71 marker expression, total RNA, and mitochondria.

Next, we exploited a virus-free CRISPR-Cas9-based editing strategy to effectively introduce genetic variants in HSPCs and successfully combined this gene-editing strategy with the erythroid differentiation protocol. We achieved $40 \%$ of precise gene modification within the beta-globin (HBB) gene, with nearly $20 \%$ of the cells harbouring the sickle mutation on both alleles. Interestingly, when incubating cells in hypoxic conditions, we were able to visualize the "sickling" phenotype of the mature and genetically modified erythrocytes.

In summary, we have successfully combined high-efficiency CRISPR-Cas9 editing of HSPCs with in vitro erythroid maturation to recapitulate sickle-cell disease in vitro. This work can be applied to other genetic variants and cell types for the study of clinically relevant mutations.

Affiliations: *Héma-Québec, ${ }^{\dagger}$ Université Laval, and ${ }^{\ddagger}$ Centre Hospitalier Universitaire de Québec Research Center, Quebec City, QC.

\section{Characterization of the shock absorbed by peripheral stem cell units during transport in cryoshippers}

Nathalie Dussault,* Lucie Boyer,* Patrick Trépanier, ${ }^{\dagger}$ Danny Brouard,* Diane Fournier ${ }^{\dagger}$

Purpose Héma-Québec provides processing, storage, and transport services for peripheral blood stem cell (PBSC) units to hospital centres in the province of Québec. Autologous cryopreserved PBSCs are brought to patients by ground transportation using cryoshippers. One incident leading to 3 PBSc bag breakages happened last year at Héma-Québec, justifying the launch of an investigation to identify the causes behind the loss of very sensitive, unique, and priceless products. One possible cause was shocks absorbed by PBSC units during ground transport in cryoshipper. To investigate this hypothesis, different bag configurations in cryoshippers (free immobilized, cassette holder) were subjected to an in-house developed drop test and to continuous shock monitoring during ground transport. Methods Four cryostore freezing bags (Origen Biomedical), filled with maximum (CS1000, CS750 and CS500) and average (CS1000) volumes of RPMI-10\% dimethyl sulfoxide (DMSO), were inserted into metal cassettes and transferred to conditioned cryoshippers. A dedicated cassette holder was designed to hold PBSC units in the cryoshipper tank and compared with the free-standing or the current vertically immobilized configurations. All cassette configurations were subjected to road transport and to repeat inhouse drop tests, including 10 vertical and side drops at increasing heights. Sterility breach observations were performed between each experiment. The cryostore freezing bag integrity was evaluated visually, and the shock amplitude profiles were recorded using a transport data logger (TDL110, Bosch). Results There were no observations of cryostore freezing bag breakage following ground transportation tests regardless of the cassette configuration in the cryoshipper tank. As shown by the shock monitoring profiles, physical stresses appear to be mainly related to the cryoshipper truck loading/unloading operations, with maximum acceleration values of [7.4 g-9.6 g]. Likewise, in the course of repeated drop tests, all freezing bags in cryoshippers maintained their integrity, irrespective of the packaging configuration. The maximum acceleration recorded during the repeated drop tests was $11 \mathrm{~g}$; this led to small external breakages at the bottom of bags without any sterility breach. The only drop-induced sterility issue was generated from a 3 -ft high lateral drop of the cryoshipper with the free-standing cassette configuration.

Conclusions Shock-induced sterility breach in PBSC bags during ground transport requires higher G-force amplitudes than what is usually observed during regular ground transport. The study results suggest that cryoshippers be handled with care during truck loading operations. The use of dedicated cassette holders to avoid movement in the cryosphipper tank improves product tolerance to shocks. Finally, the use of a transport data logger can generate valuable information, translating to a better characterization and improvements in PBSC logistics.

Affiliations: *Héma-Québec, Medical Affairs and Innovation, Quebec City, QC; ${ }^{\dagger}$ Héma-Québec, Stem Cell Laboratory, Montreal, QC.

\section{Impact of EPIC electronic medical record implementation on the Ottawa Hospital blood and marrow transplant program processes-insights and opportunities}

Carolina Cieniak,* Carey Landry,* Matthew Granger,* Melanie Trinacty, Jennifer Kuhn,* Lindsay Campbell,* Dennis Garvin,* Natasha Kekre, ${ }^{* \dagger}$ Christopher Bredeson*†

Background The Ottawa Hospital (тон) underwent an institution-wide deployment of a new electronic medical record system, EPIC, on 1 June 2019 to modernize and integrate its electronic medical record, pharmacy, laboratory, and scheduling systems. The Blood and Marrow Transplant (ВМт) program of тоH was tasked with adapting existing processes pertaining to therapeutic cell transplant coordination, collection, patient admission, treatment, supportive care, and discharge to the newly introduced platform.

Purpose To describe the planning, roll-out, and optimization used by тон BMT program to integrate existing therapeutic cell transplant coordination processes into EPIC applications and workflows, including challenges and opportunities afforded by the new system.

Methods EPIC was acquired via competitive procurement and underwent an 18-month planning period with organization-wide training, a single-day roll-out, at-the-elbow support, and a subsequent optimization period. The TOH BMT program performed an impact assessment of controlled documents, policies, staff education, and process flow for change management novel to the BMT program. A task force was developed comprised of technical project team members, managers, the program medical director, and quality coordinators to design conditioning regimen therapy plans and вMT program-specific order-sets integrate ISBT labelling and cell therapy flowsheets into the platform, to map processes on the continuum of care for identification of critical build steps, and to create and implement staff communication and training in the revised policies and procedures. The Plan, Do, Study, Act (PDSA) cycle model was utilized for continuous optimization of BMT program task integration in EPIC. A BMT staff survey was developed to assist in identification of problems and a Pareto Analysis was applied to identify key issues and process improvement opportunities. Results In the 6-month period post-launch, 114 hematopoietic cell transplants were performed at TOH (69 auto, 45 allo), exceeding the number of transplants which occurred in the preceding 6 months (55 auto, 42 allo). Four incidents were assigned to the BMT program specific to new system issues, with none attributed to adverse medical events. The biggest sources of error reported by вMT staff pertained to lab ordering and staff training. Within the first 8 weeks post go-live, all critical program forms and treatment plans were evaluated and functioning as expected, with required modifications pushed to production. More work is needed to fully integrate paper documentation.

Conclusions Implementation of a new hospital electronic medical record system is a massive undertaking. With multidisciplinary planning, application of quality management principles, quick identification of inefficiencies and optimization of processes, the program was able to safely transition patients through this complex care pathway without reduction in service or volumes.

Affiliations: *The Ottawa Hospital Cancer Program and ${ }^{\dagger}$ The Ottawa Hospital, Department of Medicine, Ottawa, ON.

\section{A systematic review of controlled preclinical trials of ECFCs and their derivative products}

Gary Liao, ${ }^{* \dagger}$ Katina Zheng, ${ }^{*}$ Risa Shorr, ${ }^{\dagger}$ David S. Allan*t

Background colony-forming cells (ECFCs) are considered "true" vascular progenitors. Their robust proliferative and self-renewal capacity make them of particular interest for the potential development regenerative therapies targeting diseases with vascular etiologies. Here we present a systematic review of controlled, preclinical trials utilizing ECFCs and/or ECFC-derived products such as conditioned media and extracellular vesicles.

Methods A systematic search of MEDLINE and EMBASE returned 3131 unique entries which were screened, 442 full-text articles were assessed for eligibility and 67 articles fulfilled the inclusion criteria.

Results The qualitative aspects of our review are consistent with narrative articles describing the state of research in this field. We show that the majority of trials involving ECFCs utilize cells derived either from umbilical cord blood or adult peripheral blood that is subsequently expanded. All studies conducted their trials in either mice (52 studies) and/or rats (16 studies) with moderate heterogeneity in their genetic background. The majority of disease models studied were in relation to ischemia with the two most commonly studied organ systems being peripheral vascular (29 studies) and the central nervous system (14 studies) followed by connective tissue (10 studies) and cardiovascular ( 7 studies). The most commonly studied disease models were hindlimb ischemia and ischemic stroke. Studies and their 
outcomes reported were heavily skewed towards positive results, with only thirteen studies reporting one or more outcomes with null results and only three studies reporting only null results, only one study reported harm. In total sixty-three studies reported at least 1 positive outcome. An assessment of study quality using the SYRCLE tool found a potential risk of bias rating of unclear risk ( $30 \%$ of the studies) and high risk of potential bias $(70 \%$ of studies). Additionally, we report on the inconsistent and heterogenous characterization of ECFC phenotypes utilized in studies Over $90 \%$ of articles utilized CD31 as a positive marker and CD45 as a negative marker. In $73 \%$ of studies, CD14 was characterized as a negative marking; CD105 was reported in only 35\% of studies and CD146 in $42 \%$. Conclusions Preclinical studies using ECFCs are associated with benefit across a range of ischemic conditions in animal models, although combining results is limited by marked heterogeneity in study design. In particular, characterization of ECFCs varied and aspects of reporting introduced potential risk of bias in most studies. More studies with greater focus on standardized cell characterization and consistent aspects of the disease model are needed.

Affiliations: *Faculty of Medicine, University of Ottawa, and ${ }^{\dagger}$ Clinical Epidemiology and Regenerative Medicine, The Ottawa Hospital Research Institute, Ottawa, ON

\section{Willingness of volunteers from Canadian Blood Service's} stem cell registry to donate blood, marrow, and other tissues for regenerative therapy

Gary Liao, ${ }^{* \dagger}$ Karen Gilmore, ${ }^{\ddagger}$ Anthony Steed, ${ }^{\ddagger}$ Heidi Elmoazzen, ${ }^{\ddagger}$ David S. Allan*t‡

Background As research surrounding cell-based regenerative therapy advances towards human trials, greater demand for cell products sourced from healthy donors will arise. The extent to which volunteers in the Canadian Blood Services Stem Cell Registry would be willing to donate cells to support regenerative therapy is not known and warrants exploration. Methods We conducted a Web-based survey to assess factors that would influence donor willingness to donate various tissues (blood, skin, fat, and bone marrow) for regenerative therapy. The survey was provided to 15,000 randomly selected donors who registered between 2013 and 2018 . Data from the 1118 respondents were analyzed.

Results Despite a mixed degree of familiarity with regenerative medicine, potential donors were very supportive of donating for direct patient care and for research and increasing their familiarity by reading a brief paragraph of information on regenerative medicine increased willingness to donate. Canadian Blood Services' stem cell registrants greatly preferred supporting non-profit groups in research and development in comparison with entities that represent profit-seeking industry involvement. The most important factors influencing willingness to donate were having an impact on patients, safety of donation, advancing knowledge in regenerative medicine, a manageable time commitment, and tolerable pain that could be managed. Donors were most willing to donate blood and had mixed responses to donating other tissue types.

Conclusions Adult volunteers from a national Stem Cell Registry are willing to support donation of biospecimens for regenerative therapy

Affiliations: *Clinical Epidemiology and Regenerative Medicine, The Ottawa Hospital Research Institute, and 'Faculty of Medicine, University of Ottawa and ${ }^{\ddagger}$ Canadian Blood Services, Ottawa, ON.

\section{Quality of life of patients and their caregivers undergoing outpatient autologous stem cell transplantation compared with inpatient transplantation}

Vinita Dhir,* Harminder Paul,* Osvaldo Espin-Garcia, ${ }^{+}$Sita Bhella,* Christine Chen,* Michael Crump,* Robert Kridel,* Vishal Kukreti,* John Kuruvilla,* Donna Reece,* Rodger Tiedemann,* Suzanne Trudel,* Anca Prica*

Background Outpatient autologous stem cell transplantation (ASCT) has become standard in many centres, with family members/friends assuming some of the patient care responsibilities. Although this may be associated with reduced direct medical costs, little work has been done to ascertain the "out of pocket costs" and "lost opportunity costs." Additionally, outpatient ASCT is perceived to provide superior quality of life (QOL) for patients, but little evidence exists. In addition, there is limited data on caregivers' QoL. Purpose Our objectives were to compare the QoL of patients and their caregivers undergoing outpatient and inpatient ASCT, and to quantify indirect costs to them.

Methods This is a single-centre study of patients with lymphoma and plasma cell disorders undergoing ASCT. Patients without a primary caregiver were still eligible to complete the QoL portion of the study. All patients completed 4 questionnaires: FACT-BMT, FACT-F, EQ-5D-3L, and a distress impact thermometer. Clinically meaningful differences between the groups, and serially, were defined as $\geq 4$ points on the FACT-BMT and FACT-F, and $\geq 0.08$ on the EQ-5D-3L.

Caregivers completed 3 questionnaires: Caregiver Quality of Life IndexCancer, distress impact thermometer, and a caregiver self-administered financial expenditure survey. Questionnaires were completed at D0 (baseline), $\mathrm{D}+7, \mathrm{D}+14$ (discharge), $\mathrm{D}+28$, and $\mathrm{D}+100$

Results From April 2016 to December 2019, 84 patients were enrolled (37 inpatients and 47 outpatients), and 61 caregivers (19 inpatients and 42 outpatients). Median patient age was 57 years (range: $18-71$ ). Of the 86 patients, $65 \%$ had a diagnosis of multiple myeloma and $35 \%$ lymphoma. The majority of caregivers were spouses (74\%).

In the overall sample, FACT-F scores (fatigue) increased at D+7, D+14 and $\mathrm{D}+28(p<0.05$ and clinically meaningful), with improvement at $\mathrm{D}+100$ [Table 1 Changes in QoL scores from baseline (D0) in the overall patient population (https://www.cttcanada.org/resource/resmgr/2020_conference/ abstracts/12.PNG)]. Compared with inpatients, outpatients had higher fatigue levels at D+7 and D+14 that were both statistically and clinically significant. For all patients, transplant-specific QOL (FACT-BMT) declined at $\mathrm{D}+7$, but then improved to above baseline values at $\mathrm{D}+100$ (both $p<0.05$ ). As per the EQ-5D-3L, best imaginable health status decreased significantly at $\mathrm{D}+7$ and $\mathrm{D}+14$ (both $p<0.01$ ), though there were no differences between the settings [Figure 1 Serial trends in QoL scores for patients (https://www. cttcanada.org/resource/resmgr/2020_conference/abstracts/13.PNG)].

With respect to caregivers, in the entire sample, QoL improved significantly by $\mathrm{D}+100$ relative to baseline $(p<0.01$, Figure 2 Serial trends in QoL scores and lost opportunity costs for caregivers (https://www.cttcanada. org/resource/resmgr/2020 conference/abstracts/14_1.PNG)]. No differences were observed between the 2 groups. The average lost wages were $\$ 4313$. The out-of-pocket costs varied, with an average of \$397 at D0 and $\$ 109$ at $\mathrm{D}+28$.

Conclusions There was significant deterioration of various QoL measures in all patients. Outpatients, however, reported significantly higher levels at $\mathrm{D}+7$ and D+14. Caregiver QoL appears comparable between the two settings and appears to improve significantly by $\mathrm{D}+100$. The financial burden on caregivers, mostly driven by lost wages is high, with a trend towards higher burden for outpatients.

Affiliations: *Division of Medical Oncology and Hematology, and ${ }^{\dagger}$ Department of Biostatistics, Princess Margaret Cancer Centre, Toronto, ON.

\section{Phase 2 trial of haploidentical allogeneic stem cell} transplantation utilizing mobilized peripheral blood stem cells Sophia Balderman,* Maureen Ross, * Christine Ho,* George Chen,* Meriem Said,* Meghan Herr,* Alan Huston,* Theresa Hahn,* Philip McCarthy*

Background Human leucocyte antigen (HLA)-mismatched haploidentical transplantation is often considered for patients lacking a suitably matched donor. Retrospective data suggest that haploidentical transplant is associated with a similar overall survival (os) rate compared with matched unrelated donor (MUD) transplants. Currently, it is standard practice in North America to use bone marrow as the source of hematopoietic stem cells (HSCs) when utilizing a haploidentical donor. Several single-centre trials and retrospective studies, however, have found that patients receiving haploidentical transplants could safely receive mobilized peripheral blood stem cell (PBSCs). Utilization of PBSCs as the source for haplo-HSCs is appealing since it obviates the need for donors to undergo general anesthesia and it results in a higher yield of CD34+ cells. We hypothesize that the use of PBSCs for patients receiving haploidentical transplants using reduced-intensity conditioning (RIC) and post-transplant cyclophosphamide (PT-Cy) will result in a reduced relapse rate as compared with the use of bone marrow as the source of HSCs. We expect that there will not be a significant increase in the rate of severe acute or chronic graft-versus-host disease (GVHD).

Endpoint To evaluate the rate of relapse at 1 year in patients who receive haploidentical PBSCs after RIC and PT-Cy.

Secondary Endpoints To evaluate safety including development of acute GVHD at 100 days post-transplant, chronic GvHD at 1 year post-transplant, and engraftment rate at 1 year post-transplant; and clinical response including os at 1 year post-transplant, treatment-related mortality (TRM) at 1 year post-transplant, and progression-free survival (PFS) at 1 year from transplant.

Correlative studies will include serial measurements of myeloid and lymphoid chimerism, and immune reconstitution.

Methods Patientsaged 1-80 years with an available 5/10-7/10 HLA-matched related donor who have high-risk hematologic malignancies, marrow failure disorders, and other nonmalignant hematologic or immunologic disorders that require transplantation, who have adequate organ function, are eligible 
for enrolment in the study. Patients will receive a conditioning regimen consisting of fludarabine, cyclophosphamide, and total body irradiation. GvHD prophylaxis will be with PT-Cy, sirolimus, and mycophenolate. Tocilizumab will be given for cytokine release syndrome (CRS) prophylaxis. Treatment will take place in the inpatient setting. If 4 of the first 15 patients enrolled in the CR arm or if 7 of the first 15 patients enrolled in the non-CR arm relapse within 1 year post-transplant, the study will be discontinued.

Sample Size A size of 30 prospective patients in the CR arm provides $80 \%$ power to detect a $15 \%$ difference in relapse compared with historical controls (10\%). A size of 28 prospective patients in the non-CR arm will achieve $80 \%$ power to detect a $20 \%$ difference in relapse compared with historical controls (50\%).

Enrolment Nine patients have been enrolled in the CR arm and 9 patients have been enrolled in the non-CR arm.

Affiliations: *Roswell Park Comprehensive Cancer Center, Buffalo, NY, U.S.A.

\section{Prospective pilot study on Frailty and Functionality assessment during pre-transplant evaluation in allogeneic hematopoietic cell transplantation}

Maria Queralt Salas, ${ }^{* \dagger \neq}$ Arijit Naq, ${ }^{* \dagger}$ Eshetu G. Atenafu, ${ }^{\ddagger}$ Ora Bascom, ${ }^{\dagger}$ Leeann Wilson, ${ }^{+}$Wilson Lam, ${ }^{* \dagger}$ Arjun Datt Law, ${ }^{* \dagger}$ Ivan Pasic, ${ }^{* \dagger}$ Dennis (Dong Hwan) Kim, ${ }^{* \dagger}$ Fotios V. Michelis, ${ }^{*+}$ Zeyad Al-Shaibani, ${ }^{* \dagger}$ Armin Gerbitz, ${ }^{* \dagger}$ Auro Viswabandya, ${ }^{* \dagger}$ Jeffrey Howard Lipton, ${ }^{* \dagger}$ Jonas Mattsson, ${ }^{*+}$ Shabbir M.H. Alibhai, ${ }^{\ddagger}$ Rajat Kumar*†

Introduction A Frailty and Functionality assessment was designed at our centre to be implemented using existing resources.

Purpose We aim to share the experience implementing this evaluation and, correlate results with post-transplant outcomes.

Methods Frailty and Functionality assessment has become part of the standard of care at our centre since July 2018. The evaluation is done at first consultation and across all ages. The assessment consists of a Clinical Frailty Scale (CFS), Instrumental Activities of Daily Living scale, grip strength Timed Up and Go Test (TUGT), self-rated health (SRH), single question of falls, and albumin and C-reactive protein (CRP) level.

All patients provided informed consent. Data were analyzed prospectively and updated in November 2019. Median follow-up was 5.3 months (range: 0.7-14.8). A univariate Cox regression analysis was done to correlate results provided by the Frailty and Functionality assessment with overall survival (OS) The impact of this evaluation in non-relapse mortality (NRM) was explored with Grey test.

Results Between July 2018 and September 2019, 280 patients were evaluated. Median time required to perform the entire evaluation ranged between 5-6 minutes. Less than 5\% of the parameters were missed and mostly during early implementation.

The impact of the evaluation in post-transplant outcomes was analyzed in 168 consecutive patients who underwent transplantation. Main baseline characteristics: median age was 58 years (range: 19-76), 95 (56\%) patients were previously diagnosed with acute myeloid leukemia, $38(23.4 \%)$ had a Karnofsky performance status between $70 \%-80 \%, 54(32.1 \%)$ an HCT-C score $\geq 3$ and $115(68.4 \%)$ received reduced intensity conditioning regimen

Mean time of transplant hospitalization was 28 days. SRH score B.C.D.E and raised CRP level were significant predictors for longer mean transplant-hospitalization $(p<0.05)$. No parameter correlated with an increased risk of readmissions or cumulative incidence of GVHD.

A TUGT $>10$ seconds (HR: $2.9 ; p=0.003$ ), raised CRP (HR: $4.4 ; p<0.001$ ) and hypoalbuminemia (HR: $2.1 ; p=0.043$ ) were significant risk factors for worse OS; CFS $\geq 3$ (HR: $3.1 ; p=0.009$ ), TUGT $>10$ seconds (HR: $3.47 ; p=0.003$ ), abnormal grip strength test (HR: $2.56 ; p=0.029$ ); SRH score B.C.D.E (HR: 3.8 ; $p<0.001$ ), elevated CRP (HR: $11.7 ; p<0.001$ ), and hypoalbuminemia (HR: $4.6 ; p<0.001$ ) were significant predictors for worse NRM.

On multivariable analysis, TUGT $>10$ seconds and raised CRP were significant predictors for worse os and NRM; SRH (less than excellent) was found to be a significant predictor for higher NRM.

Conclusions With selected tools, Frailty and Functionality can be assessed as part of routine clinical practice in alloHCT without extra waiting time for patients, additional human resources or recurrent costs.

Larger number of patients, longer follow-up and multicentre validation will help to demonstrate the true potential of this evaluation.

Affiliations: *University of Toronto, Department of Medicine, Section of Medical Oncology and Hematology, ${ }^{\dagger}$ Hans Messner Allogeneic Blood and Marrow Transplantation Program, Division of Medical Oncology and Hematology, Princess Margaret Cancer Centre, University Health Network, and ${ }^{\ddagger}$ Department of Biostatistics, Princes Margaret Cancer Centre, University Health Network, Toronto, ON

\section{Dual T cell depletion using ATG and PTCy for GvHD prophylaxis in RIC alloHCT using in 10/10 matched related and unrelated donors: largest single-centre experience}

Maria Queralt Salas, ${ }^{*+\ddagger}$ Eshetu G. Atenafu, ${ }^{\S}$ Arjun Datt Law, ${ }^{* \dagger}$ Wilson Lam, ${ }^{*+}$ Zeyad Al-Shaibani, ${ }^{* \dagger}$ Ivan Pasic, ${ }^{*+}$ Dennis (Dong Hwan) Kim, ${ }^{* \dagger}$ Fotios V. Michelis, ${ }^{* \dagger}$ Armin Gerbitz, ${ }^{* \dagger}$ Jeffrey Howard Lipton, ${ }^{* \dagger}$ Jonas Mattsson, ${ }^{* \dagger}$ Rajat Kumar, ${ }^{* \dagger}$ Auro Viswabandya*t

Introduction The use of anti-thymocyte globulin (total dose $4.5 \mathrm{mg} / \mathrm{kg}$ ), post-transplantation cyclophosphamide, and cyclosporine (ATG-PTCy-CsA) for graft-versus-host disease (GVHD) prophylaxis in reduced-intensity conditioning (RIC) alloHCT was implemented at our centre in 2015. Secondary to infectious complications, the total dose of ATG was lowered to $2 \mathrm{mg} / \mathrm{kg}$ in May 2018.

Purpose We aim to compare the efficacy and safety of this GvHD prophylaxis among both ATG doses in RIC alloHCT using 10/10 matched sibling donor (MSD) and matched unrelated donor (MUD)

Methods Among the 250 adults included, $175(70 \%)$ received $4.5 \mathrm{mg} / \mathrm{kg}$ of rabbit-ATG (day $-3,-2,-1)$, and $75(30 \%)$ received $2 \mathrm{mg} / \mathrm{kg}$ (day $-2,-1)$. Data were updated in October 2019. The cumulative incidence (cum.inc) of GVHD was calculated using death and relapse as competing events.

Results Median age was 59 years (range: 18-74), 77 (31.8\%) patients received MSD grafts and $275(69.2 \%)$ received MUD grafts. Baseline characteristics were balanced between both cohorts $(p>0.1)$, except the proportion of patients with aKarnofsky performance status of $70 \%-80 \%$, which was higher in the group who received $2 \mathrm{mg} / \mathrm{kg}$ of ATG $(p=0.001)$.

The reduction of the ATG dose did not have a significant impact on the cum.Inc of clinically relevant GvHD ( $p>0.1)$. The cum.inc of grade II-IV and III-IV aGvHD at day +100 and moderate/severe cGvHD at 1 year in patients receiving $4.5 \mathrm{mg} / \mathrm{kg}$ was $12.5 \%, 4 \%$, and $12.5 \%$ respectively; and among patients who received $2 \mathrm{mg} / \mathrm{kg}$ was $17.4 \%, 3.5 \%$, and $17.4 \%$. The cum.inc of clinically relevant GvHD was comparable across both donor types $(p>0.1)$.

Proportions of cytomegalovirus and Epstein-Barr virus reactivation were comparable among both cohorts $(p>0.1)$. Seventeen $(6.8 \%)$ patients had PTLD, and one received a total ATG dose of $2 \mathrm{mg} / \mathrm{kg}(p=0.06)$. The proportion of patients with other viral infections was lower in patients who received $2 \mathrm{mg} / \mathrm{kg}$ of ATG $(p=0.002)$.

The median follow-up of the entire cohort, patients who received $4.5 \mathrm{mg} / \mathrm{kg}$ of ATG, and $2 \mathrm{mg} / \mathrm{kg}$ of ATG was respectively: 14 (range: 0.4-47), 19 (0.5-45), and $9(0.4-15)$ months. Overall, 79 (31.6\%) patients died and $59(23.6 \%)$ relapsed. Main causes of death were infection (15\%) and relapse (14\%). Oneyear overall survival (OS), relapse-free survival (RFS), non-relapse mortality (NRM), cumulative incidence of relapse (CIR), and GVDH-free and relapse-free survival (GRFS) were, respectively, $71.7 \%, 65.2,14.9 \%, 19.8 \%$, and $53.7 \%$.

The impact of the reduction of the ATG dose on OS, RFS, NRM, CIR, and GRFS was not statistically significant on the univariate analysis (Cox regression/ Fine-Grey, $\mathrm{p}>0.1$ ). The multivariable analysis confirmed that the administration of $2 \mathrm{mg} / \mathrm{kg}$ of ATG provided a nonsignificant protective effect on NRM (HR: $0.48 ; 95 \%$ CI: 0.2 to 1.01$) ; p=0.056$ ), and that the reduction of the ATG dose did not have a significant impact on OS, RFS, GRFS $(p>0.1)$.

Conclusions RIC alloHCT combined with ATG-PTCy-CSA is a safe protocol for adults undergoing alloHCT and effectively controls GvHD. Dual T cell depletion with ATG and PTCy provides an impressive control of GvHD with comparable relapse rates using peripheral blood stem-cell grafts. The reduction of ATG to $2 \mathrm{mg} / \mathrm{kg}$ decreased NRM providing comparable control of acute GvHD. Longer follow-up is needed to confirm the efficacy of the refined protocol in controlling chronic GVHD.

Affiliations: *University of Toronto, Department of Medicine, Section of Medical Oncology and Hematology, Toronto, ON; ${ }^{\dagger}$ Hans Messner Allogeneic Blood and Marrow Transplantation Program, Division of Medical Oncology and Hematology, Princess Margaret Cancer Centre, University Health Network, Toronto, ON ‡Hematology Department, Institut Català d'Oncologia-Hospitalet, Barcelona, Spain; ${ }^{\S}$ Department of Biostatistics, Princes Margaret Cancer Centre, University Health Network, Toronto, ON.

\section{Short-term exposure of umbilical cord blood CD34+ cells to human platelet lysate and cytokines enhances engraftment}

Marie-Ève Rhéaume, ${ }^{*}$ Lionel Loubaki, ${ }^{* \dagger}$ Tony Tremblay,* Isabelle Paré, Pascal Rouleau*

Background Umbilical cord blood (UCB) is a widely used source of stem cells in therapies of malignant and nonmalignant hematologic diseases and metabolic disorders. However, UCB grafts are limited by low numbers of total and stem cells that are associated with delayed hematopoietic and immunologic recovery. Intra-bone marrow (IBM) injection has been proposed as a strategy to bypass homing inefficiencies associated with intravenous (IV) hematopoietic stem-cell (HSC) transplantation and to increase the number of HSC that engraft. Despite physical delivery into the 
Bм cavity, many donor cells are rapidly redistributed by vascular perfusion, thus potentially compromising the efficacy of this approach.

Purpose The objective of our study was to evaluate the ability of human platelet lysates (hPL) to improve HSC anchorage into the BM and consequently to improve engraftment.

Methods The EasySep Human CD34-Positive Selection Kit (STEMCELL Technologies) was used to isolate HSC, and purity was assessed by flow cytometry. The HSC were then seeded in the wells of a 24 -well microplate and exposed to increasing concentrations of hPL with or without cytokines for $24 \mathrm{~h}$ at $37^{\circ} \mathrm{C}, 5 \% \mathrm{CO}_{2}$. Following culture, HSC cells chemotaxis to rhSDF- 1 was determined in vitro using a Transwell and engraftment in NSG mice was also evaluated.

Results Short-term exposure of cord blood CD34+ cells to a combination of human platelet lysates (hPL) and cy tokines resulted in a significant increase (up to 3 -fold) in the expression of CD34 antigen on HSC that was closely correlated with a significantly increased migration towards a gradient of rhSDF-1 (up to 7-fold). In addition, IBM injection of CD34+ cells previously exposed to hPL+cytokines into NSG mice (Jackson Laboratory, Bar Harbor, ME, U.S.A.) showed significantly increased engraftment, as measured by human platelet numbers $(704.9 \pm 230.3$ vs. $3193.0 \pm 1806.7$ human platelets (hPLt) $/ \mathrm{uL}$ of blood, $p=0.005$ ), human CD $4513.2 \% \pm 4.4 \%$ vs. $22.2 \% \pm 1.4 \%$ ) and human CD34+ cells $(2.4 \% \pm 1.2 \%$ vs. $6.6 \% \pm 1.1 \%)$ for untreated and treated cells respectively.

Conclusions The use of hPL + cytokines as a short-term priming treatment for UСв could be an attractive strategy to improve clinical outcomes following Івм injection.

Affiliations: *Medical Affairs and Innovation, Héma-Québec, ${ }^{\dagger}$ Department of Biochemistry, Microbiology and Bioinformatics, Laval University, Québec, QC.

32. Improved access to better HLA-matched hematopoietic cells for allogeneic transplant: analysis of donors and cord blood units selected for Canadian patients in $\mathbf{2 0 1 8}$

Valerie Greco-Stewart, ${ }^{*}$ Heidi Elmoazzen, ${ }^{*}$ Gail Morris, ${ }^{*}$ Yiming Guo,* Colleen Langdon,* Dena Mercer,* Nicholas Dibdin,* David Allan*t+

Background Patients undergoing hematopoietic cell transplantation (НСT) often require use of an unrelated donor or cord blood unit (СBU). An understanding of evolving practices in graft selection is needed for optimization of donor recruitment and cord blood collection.

Study Design and Methods Each donor workup requested in 2018 involving a Canadian patient and unique donor product or СвU was reviewed $(n=598)$. Degree of human leucocyte antigen (HLA) match, product origin (domestic or international), and non-HLA factors including donor age, sex, cytomegalovirus (CMV), and ABO compatibility were analyzed for workups that proceeded to transplant $(n=414)$. We also analyzed changes compared with a similar analysis performed in 2013 .

Results The majority of transplants used matched unrelated donors [matched unrelated donor (MUD), $n=323,78 \%$ ] and were most often young ( $<35$ years), male, international donors $(n=136)$. The proportion of transplants involving MUDs, as opposed to mismatched unrelated donors or CBU, increased by $12.4 \%$ compared with 2013 . When young, male, Canadian MUDs were identified in patient search reports but not selected, CMV mismatching and ABO incompatibility were most likely to have influenced the decision to use an international MUD. Consistent with global trends, CBU transplants decreased compared with 2013. However, the degree of HLA matching improved significantly, and $27 \%$ of transplanted CBUs were procured from the Canadian Blood Services Cord Blood Bank.

Conclusions Access to MUD and better HLA-matched CBUs by Canadian patients has increased since 2013. Ongoing recruitment of young registrants and cord blood donors with diverse HLA haplotypes will support selection of donors with optimal non-HLA characteristics.

Affiliations: *Stem Cells, Canadian Blood Services, ${ }^{\dagger}$ The Ottawa Hospital Research Institute, and ${ }^{\ddagger}$ Department of Medicine, University of Ottawa, Ottawa, ON.

\section{Factors associated with registrant availability for unrelated adult donor hematopoietic stem cell donation: analysis of the Stem Cell Registry at Canadian Blood Services}

Madeleine Monaghan, ${ }^{*}$ Qi-Long $\mathrm{Yi}_{1}{ }^{+}$Meaghan Green,, Todd Campbell, Jason Weiss, ${ }^{\ddagger}$ Nicholas Dibdin, ${ }^{\neq}$Dena Mercer, ${ }^{\ddagger}$ Heidi Elmoazzen, ${ }^{\ddagger}$ David Allan*‡\$

Background Greater use of unrelated donors to support hematopoietic cell transplantation (HCT) can be hampered by unavailability of registrants when identified as potential candidates for donation.

Methods Multivariate analysis was performed to identify donor factors associated with availability for verification of human leucocyte antigen typing (VT) needed prior to donor activation. All vT requests for registrants on Canadian Blood Services' Stem Cell Registry between 1 January and 31 December 2018 were reviewed $(n=1358)$.

Results Potential donors identified by transplant centres were categorized as available at time of VT but ineligible due to medical or other reasons ( $n=130$ and excluded from further analysis), available ( $n=622)$, or unavailable ( $n=566)$ due to scheduling, loss of interest, and/or inability to contact. With multivariate analysis, registrants who previously donated blood, those recruited online or from blood donation clinics, and a shorter interval between registration and VT request were significantly correlated with increased donor availability. Donor sex and geographic location, however, displayed no correlation.

Conclusions Online registration and recruitment at whole blood donation centres should be enhanced to increase the availability of registrants at VT. More insight is needed to maintain registrant availability following community in-person recruitment events, especially if the interval between registration and activation is prolonged. Recruitment of male registrants who are well informed should not negatively impact availability.

Affiliations: *Clinical Epidemiology, Ottawa Hospital Research Institute, ${ }^{\dagger}$ Epidemiology and Surveillance, ${ }^{\ddagger}$ Stem Cells, Canadian Blood Services, and ${ }^{\S}$ Faculty of Medicine, University of Ottawa, Ottawa, ON.

34. Comprehensive analysis of methods and outcome reporting in preclinical animal studies of mesenchymal stem cell-derived extracellular vesicles: a systematic review

Alvin Tieu, ${ }^{* \dagger \neq}$ Manoj M Lalu, ${ }^{* \dagger \neq \S \|}$ Duncan J. Stewart, ${ }^{* \neq||}$ Mitchell

Slobodian, ${ }^{\dagger}$ Dean A. Fergusson, $+\| \#$ Joshua Montroy, ${ }^{+}$Dylan Burger,***+† David S. Allan*t+\|

Background Mesenchymal stem cell-derived extracellular vesicles (MSC-EVs) can modulate cell processes to reduce inflammation, promote healing, and improve organ function thereby providing a potential "cellfree" therapeutic product. Prior to translating Ev therapy to clinical trials, there is a critical need to synthesize the existing preclinical evidence supporting their efficacy. This systematic review aims to provide the most comprehensive evidence map of methods, safety, and efficacy for MSC-EV research to date.

Methods MEDLINE and EMBASE were systematically searched for preclinical, in vivo interventional studies using MSC-EVs as a therapy. Two reviewers extracted data in duplicate for methods, experimental design, interventional traits, and efficacy or adverse events.

Results After screening 754 articles, 206 studies met our eligibility criteria. A wide variety of diseases, including renal (16\%), neurologic (12\%) and cardiac (10\%) conditions were treated using MSC-EVs. Overall, benefits were described in $95 \%$ of studies across all organ systems and adverse effects were reported in only three studies; two showing tumour growth. However, several key methodology concerns were evident. Based on size criteria for EV subtypes (exosomes/small Evs $\sim 30-150 \mathrm{~nm}$, microvesicles $\sim 150-1000 \mathrm{~nm}$ ) only $60 \%$ of studies used appropriate nomenclature. Ultracentrifugation $(70 \%)$ and isolation kits (23\%) were the most common isolation methods despite their marked differences in yield and purity. The EVs were inconsistently dosed by protein amount $(68 \%)$, particle number $(16 \%)$, or MSC cell count (4\%), limiting the ability for inter-study comparisons. Two thirds of studies used xenogeneic EVs suggesting that they are immunocompatible. Techniques to determine size, protein markers, and morphology was highly heterogeneous, and only twelve and four studies met the characterization standards recommended by the Minimal information for studies of EVs (MISEV) 2014 and 2018 guidelines respectively. Finally, 50\% of studies did not incorporate randomization which represents a high risk for bias and only a quarter of studies performed biodistribution experiments.

Conclusions This systematic review of animal studies of MSC-EVs reveals extensive heterogeneity in methodology and interventional details. Nonetheless, almost all studies showed significant benefits in a wide range of distinct conditions. The knowledge gaps we have identified highlight important opportunities for improving preclinical design and the need for more standardized approaches in this growing field of EV therapeutics.

Affiliations: *Department of Cellular and Molecular Medicine, University of Ottawa; ${ }^{\dagger}$ Clinical Epidemiology Program, BLUEPRINT Translational Research Group, The Ottawa Hospital Research Institute; ${ }^{\ddagger}$ Regenerative Medicine Program, The Ottawa Hospital Research Institute; ${ }^{\S}$ Departments of Anesthesiology and Pain Medicine, "Medicine, "Surgery, and **Nephrology, University of Ottawa, The Ottawa Hospital; and ${ }^{\dagger \dagger}$ Kidney Research Centre, The Ottawa Hospital Research Institute, Ottawa, ON.

35. Cost-effectiveness analysis of allogeneic versus autologous stem cell transplant versus chemoimmunotherapy for early relapsed follicular lymphoma

Abi Vijenthira,* John Kuruvilla,* Anca Prica ${ }^{\dagger}$ 
Purpose Among patients with follicular lymphoma requiring treatment, $20 \%$ develop progressive disease within 2 years and have a 5 -year overall survival of $50 \%$. The purpose of this study was to compare the qualityadjusted effectiveness and costs of 3 treatment options [allogeneic (alloscT) or autologous stem cell transplant (АSCT), or chemoimmunotherapy] for patients eligible for transplant with early relapse of follicular lymphoma Methods A Markov decision-analytic model was developed using a 20year time horizon. The treatment options of alloscT versus ASCT versus chemoimmunotherapy (O-CHOP) were compared for patients eligible for transplant with early relapsed follicular lymphoma. For relapsed/ refractory disease, novel therapy options including lenalidomide and rituximab/obinutuzumab (AUGMENT, MAGNIFY, GALEN trials), and tyrosine kinase inhibitors [TKIs-duvelisib (DYNAMO), copanlisib (CHRONOS)] were included in the treatment algorithm. Baseline probabilities in the model included the risks of treatment-related mortality, relapse, graft-versushost-disease (GVHD), and secondary malignancy. Baseline utilities in the model included utilities related to each treatment regimen, of relapse, GVHD, secondary malignancy, being on novel treatments, and having end-stage follicular lymphoma. Estimates were derived from a systematic review of published data. Costs were considered from a Canadian public health payer's perspective.

Results Probabilistic analyses (10,000 simulations) demonstrated that, at a willingness-to-pay threshold of $\$ 50,000$, an ASCT strategy was more cost-effective $60 \%$ of the time [Figure 1 (https://www.cttcanada.org/ resource/resmgr/2020_conference/abstracts/15.PNG)]. Compared with the other strategies, ASCT resulted in a higher number of both life-years (ASCT 10.2 vs. allosCT 9.9 vs. O-CHOP 10.0 years) and quality-adjusted lifeyears (ASCT 7.5 vs. allosCT 6.6 vs. O-CHOP 7.4 years), with the lowest direct costs $(C A \$ 190,128)$. Sensitivity analyses demonstrated that the model was robust to key variables, including differing relapse probabilities, probabilities of mild and severe GvHD, cost of treating GVHD, cost of TKIs, and the probability of secondary malignancy from ASCT. However, when the life expectancy of the patient was less than 10 years or the patient was older than 65 years, chemoimmunotherapy was the preferred treatment strategy at a willingness-to-pay of $\$ 50,000$ [Figure 2 (https://www.cttcanada.org/ resource/resmgr/2020_conference/abstracts/15.PNG)].

Conclusions Our results suggest that when considering cost, effectiveness, and short- and long-term toxicities, the preferred treatment strategy for most patients with early relapsed follicular lymphoma is ASCT. Although our findings do not provide an absolute best treatment approach for all patients, they can contribute to shared decision-making between patients and treating physicians.

Affiliations: *Postgraduate Medical Education, University of Toronto, and ${ }^{\dagger}$ Prin cess Margaret Cancer Centre, Toronto, ON.

36. Continuous alloreactive $\mathrm{T}$ cell depletion and regulatory $\mathrm{T}$ cell expansion for the treatment of steroid-refractory or dependent chronic GvHD—a multicentre phase 2 clinical trial (the CARE trial) Imran Ahmad, * Janetta Bijl,* Eshetu Atenafu, ${ }^{+}$Tony Panzarella, ${ }^{+}$Raewyn Broady, ${ }^{\ddagger}$ David Szwajcer, ${ }^{\S}$ Irwin Walker," Dennis Kim, ${ }^{\dagger}$ Chris Bredeson, ${ }^{\sharp}$ Ronan Foley, I Donna Hogge, ${ }^{\ddagger}$ David Allan, ${ }^{*}$ Jean-Sébastien Delisle,* Megan Levings, ${ }^{\ddagger}$ Kirk Schultz, ${ }^{\ddagger}$ Donna Wall, ${ }^{+}$Denis-Claude Roy*

Introduction Steroid-refractory and steroid-dependent chronic graftversus-host disease (SR/SD-cGvHD) are a therapeutic challenge since sustained response and tolerability to second-line systemic agents are erratic. Extracorporeal photopheresis constitutes a treatment option with an excellent efficacy/safety profile but requires prolonged and frequent apheresis sessions.

Objective We sought to determine the safety and preliminary efficacy of TH9402-based photodynamic therapy (TH-PDT), a new type of phototherapy that does not involve frequent apheresis, in the multicentre CARE trial sponsored by the Canadian Donation and Transplantation Research Program (CDTRP). Ex vivo manipulation of mononucleated cells and their reinfusion in several fractions had the advantage of using only 6 apheresis sessions over a 6 -month treatment period. Six Canadian transplant centres participated in this trial. Failure-free survival was used as primary outcome measure, with failure defined by addition of a new systemic treatment for GVHD, relapse of transplanted disease, or death. A comprehensive immunological evaluation of the cell product and patient blood specimens was embedded in the trial.

Summary Among 17 enrolled patients, no grade $>3$ adverse event (AE) was observed. Two subjects were withdrawn from the study for grade $3 \mathrm{AEs}$ unrelated to cell infusion. The failure-free survival was $72 \%$ (95\% CI: $42 \%$ to $89 \%$ ) at 24 weeks from the start of the treatment. Failure was observed in 4 subjects ( 3 addition of new treatment, 1 relapse). The overall response rate (NIH criteria) was $46 \%$, with best responses in GI tract, liver, eye and mouth involvement. Steroid dose reduction ( $\geq 50 \%$ of starting dose) was achieved in $85 \%$ patients and $46 \%$ patients were able to discontinue an additional systemic agent.

Correlation with standardized immune markers is underway and may enlighten mechanisms of response to cGvHD treatment and provide biomarkers for prediction of response.

Conclusions TH9402-based photodynamic therapy can offer a safe and effective treatment option for SR/SD-cGvHD and deserves comparison with other available treatment options.

Project funded by the Canadian Donation and Transplantation Research Program (CDTRP). Acknowledgement: Daphne Brockington (University of British Columbia), study coordinator.

Affiliations: *Université de Montréal, Montreal, QC; ${ }^{\dagger}$ University of Toronto, Toronto, ON; ${ }^{\ddagger}$ University of British Columbia, Vancouver, BC; ${ }^{\S}$ University of Manitoba, Winnipeg, MB; "McMaster University, Hamilton, ON; "University of Ottawa, Ottawa, ON.

\section{Use of B cells for specific antigen presentation to} T lymphocytes, from the perspective of adoptive immunotherapy Guillaume Bonnaure, ${ }^{* \dagger}$ Cédric Carli,* Sonia Néron, ${ }^{\dagger}$ Jean Sébastien Delisle*‡

T Lymphocytes are becoming increasingly popular in the field of immunotherapy. Modern medicine now has many tools to enhance T lymphocytes' immune functions by boosting their antigen recognition properties. One way to obtain such enhanced T cells is by using a peptide mimicking an antigen, which can intensify/improve the cells' specificity against it.

This process requires antigen-presenting cells (APCs), either dendritic cells (DCs), macrophages or B cells, which are able to present a given major-histocompatibility complex (MHC)-associated peptide at their surface. Dendritic cells are the main APCs used in cellular therapy. Adoptive immunotherapy requires a substantial number of cells, and producing enough DCs for such therapies can be quite challenging and labour-intensive.

B cells also possess APCs properties. However, the use of human B cells as a substitute for DCs, to induce $T$ cell activation in many settings remains to be determined. By using leucoreduction system chambers, we can harvest millions of B cells, with the same profile and function as those found in peripheral blood. Our research aim was to find the B cell population best capable to induce a $\mathrm{T}$ cell response to a specific antigen in vitro. In a media supplied by human serum, our results show that CD27+ memory B cells are the best B cell APCs in our model, inducing a response from $\mathrm{T}$ cells against cytomegalovirus (CMV) peptides in donors not previously exposed to the virus (seronegative) as assessed by cytokine release. Proteins involved in costimulatory signal appear to be more expressed in the memory B cell subpopulation. Furthermore, co-culture with memory B cells increase T cells expression of exhaustion markers such as LAG3 and TIM3, in comparison with naïve B cells stimulation and in accordance with their increased activation state. While activation with B cells did not outperform the results obtained with DCs, memory B cells emerged as a strong candidate to generate in vitro specific $\mathrm{T}$ cells for a given peptide. Those results open a new approach in terms of $\mathrm{T}$ cell activation in vitro, from an immunotherapy perspective.

Affiliations: *Research Centre of Maisonneuve Rosemont Hospital, Montre al, QC; †Héma-Québec, Medical Affairs and Innovation, Quebec City, QC; ${ }^{\ddagger}$ Hematology-Oncology Division, Hôpital Maisonneuve-Rosemont, Montreal, QC; ${ }^{\circledR}$ Department of Medicine, Université de Montréal, Montreal, QC.

\section{Outcomes of EBV viremia and PTLD after rituximab: a pharmaco-cost analysis}

Jack T. Seki, ${ }^{* \dagger}$ Eshrak Al-Shaibani, ${ }^{\dagger}$ Lina Ho, ${ }^{* \ddagger}$ Patwant Dhillon, ${ }^{*}$ Cassandra McEwan, ${ }^{*}$ Ian Pang, ${ }^{*}$ Eshetu Atenafu, ${ }^{*}$ Rajat Kumar ${ }^{+}$

Background Epstein-Barr virus (EBV)-related post-transplantation lymphoproliferative disorders (EBV-PTLDs) are rare but potentially fatal complications of allogeneic hematopoietic cell transplantation (allo-HCT). The current treatment strategies for EBV-PTLDs include reduction of immunosuppression (RI), rituximab, adoptive cell therapy (unselected donor lymphocyte infusion), and EBV-specific cytotoxic T lymphocyte infusion and chemotherapy, among which RI and rituximab are the mainstay. Rituximab-based pre-emptive treatment can prevent EBV viremia from developing into EBV-PTLDs. After October 2018, our institute initiated the pre-emptive treatment for EBV viremia and used a titre of 300,000 IU/mL as treatment threshold.

Purpose To determine the outcome before and after initiation of preemptive treatment of EBV-viremia and the cost associated with hospitalized vs. outpatient therapy.

Methods Pharmacy and transplant registry databases were used to identify patients who underwent allo-HCT between February 2014 and 
December 2018 and subsequently received rituximab for EBV viremia or EBV-PTLD at our institution. Data abstraction was specific for EBV polymerase chain reaction titer, clinical, PET/CT-evident PTLD, and/or pathology prior to treatment initiation. Post treatment resolution or recurrence was analyzed. The Finance Department provided cost estimates for hospitalized and outpatient treatment costs.

Results There were 713 allo-HCT performed during this period and 41 $(5.8 \%)$ received rituximab for EBV-related indication. The median age of these 41 patients was 57 (range: 23-75) with 24 men. Myeloablative conditioning was administered to $17.5 \%$ of patients while $82.5 \%$ received reduced-intensity conditioning. In vivo T cell depletion was given to $95 \%$ of patients, either anti-thymocyte globulin or alemtuzumab in 33 and 5 patients respectively. Donors were matched related in $15 \%$, unrelated in $72.5 \%$, and haploidentical in $12.5 \%$. One patient was excluded because she received rituximab for another reason. Table 1 (https://www.cttcanada org/resource/resmgr/2020_conference/abstracts/16.PNG) shows the distribution and outcome of the patients for EBV-viremia and EBV-PTLD before and after pre-emptive treatment. The overall rate of treatment success was $83 \%$ ( 34 of 41 patients); $12 \%$ (5 patients) was resistant and $5 \%$ ( 1 patient) was intolerant with infusion-related reaction. In 18 patients (44\%), rituximab was initiated as inpatient and the entire course was completed in hospital, while in 5 patients $(12 \%)$ it was given on an outpatient basis. In the remaining 18 patients (44\%) treatment began as outpatient but was completed as inpatient. The average cost of resources per cycle of treatment, including total nursing, supplies and cost of bed (inpatient) or chair (outpatient) was $\$ 1,450$ and $\$ 100$ respectively. The cost of rituximab is not factored in here. Conclusions In this retrospective study, pre-emptive therapy of EBV was effective in preventing EBV-PTLD. Outpatient treatment was more costeffective than inpatient treatment.

Affiliations: *Department of Pharmacy, Princess Margaret Cancer Centre, University Health Network, †Leslie Dan Faculty of Pharmacy, University of Toronto and ${ }^{\ddagger}$ Hans Messner Allogeneic Transplant Program, Princess Margaret Cancer Centre, Toronto, ON

39. The impact of hematopoietic stem-cell transplantation on work and productivity loss in patients with acute leukemia: results from a rapid review

Tayler Scory,* Eileen Shaw,* Tara Cowling,* François Péloquin, ${ }^{\dagger}$ Verna Welch, ${ }^{\ddagger}$ Majed Charaan, ${ }^{\dagger}$ Andrew Brown ${ }^{\ddagger}$

Background Although costly and resource intensive, hematopoietic stem-cell transplantation (HSCT) remains the only potentially curative intervention for most patients with hematologic malignancies including acute lymphocytic leukemia (ALL) and acute myeloid leukemia (AML). For these patients, work productivity outcomes are important in long-term survivorship care, with a recent European study reporting 3.6 billion in productivity losses due to hematologic malignancies.

Purpose This review aims to evaluate the impact on productivity and understand the societal burden of HSCT in patients with ALL or AML.

Methods A rapid review was conducted to identify real-world evidence reporting work and productivity loss associated with ALL and AML, including the impact of HSCT. A PubMed search was executed for reports published 1 April 2014 to 1 April 2019, in English, and without geographic restriction. Eligible studies examined outcomes on work productivity in patients diagnosed with ALL, AML, or combined hematologic malignancies. A single reviewer screened all identified citations and extracted key data from included papers.

Results Thirteen unique real-world data studies were identified. Seven studies examined the impact of hematologic cancers (including ALL and AML) on varied return-to-work outcomes. Of these, three studies observed positive return-to-work outcomes over time. Four of these studies reported decreased employment among patients compared with the general population; however, two of these focused on childhood hematologic cancer survivors. Six studies examined the impact of HSCT on work productivity. Two studies reported opposing results in unemployment in adult survivors of childhood hematologic cancers who received НSCT. The remaining four studies found a general decrease in employment or increase in low-income status among adult patients when comparing pre-HSCT to post-HSCT. Two of these studies reported that most patients who were surveyed at 1 or 5 years post-HSCT were still employed, with $62 \%$ returning to work over 1 year postHSCT in one study and $77 \%$ of patients returning to work 5 years post-HSCT in the other study. Overall, there was a trend towards work reintroduction as an important outcome post-HSCT. Recurring themes included structura changes in the current delivery of health services or potential interventions to help rehabilitation post-HSCT and optimize employment.

Conclusions Although the number of studies was limited, results indicate that while an AML or ALL diagnosis negatively affects productivity (including in HSCT recipients), a substantial proportion of patients remain actively employed. This review identified trends suggesting that longer survivorship post-HSCT increases the likelihood of a patient returning to being a productive member of society. Future research should focus on understanding the causes of unemployment post-HSCT to help prevent adverse financial outcomes following this procedure.

Affiliations: *Medlior Health Outcomes Research Ltd., Calgary, AB; ${ }^{\dagger}$ Pfize Canada Inc., Montreal, QC; ${ }^{\ddagger}$ Pfizer Inc., New York, NY, U.S.A

\section{Engaging gay, bisexual, and other men who have sex with men} to register as stem cell donors in Canada: a pilot campaign Daniel Tarade, ${ }^{* \dagger}$ Kenneth Williams, ${ }^{*+}$ Shamini Vijaya Kumar, ${ }^{*+}$ Sze Wah Samuel Chan, ${ }^{* \dagger}$ Brendon Lam, ${ }^{* \ddagger}$ Kaveh Farrokhi, ${ }^{* \S}$ Adriyan Hrycyshyn, ${ }^{* \S}$ Dylan Coupal,*\| Sylvia Okonofua, ${ }^{* *}$ Hayley Wroot,*** Kyla Pires, ${ }^{* * *}$ Sachini Jayasinghe, $* * *$ Warren Fingrut*++

Introduction Since 2009, gay, bisexual, and other men who have sex with men (MSM) have been eligible to register as stem-cell donors in Canada and donate to a patient in need; however, MSM may not be aware of their eligibility. Targeted recruitment of MSM could augment efforts to recruit the most needed stem cell donors (young and ethnically-diverse men).

Purpose Here, we describe a strategy for targeted recruitment of MSM as stem-cell donors and outcomes of a pilot campaign spearheaded by the Canadian donor recruitment organization Stem Cell Club.

Methods Experienced donor recruiters from Stem Cell Club were invited to run stem-cell drives at Pride events, targeting recruitment of MSM as stem-cell donors. Prior to these drives, participating recruiters completed a 90-minute workshop on blood and stem-cell donation for MSM in Canada. The workshop coached recruiters to answer questions such as "What are the policies for MSM blood donation and stem-cell donation in Canada?" "Why does the stem cell registration form ask about MSM?" "I am a Trans* person, how should I fill out the paperwork to register as a donor?" and "I am HIV-positive and I am healthy, can I sign up as a stem cell donor?" Recruiters were also taught to share the story of Ashby-Hall, a gay man who donated his stem cells to an unrelated Montreal-based patient [CBC News, 2019; Figure 1 (https://www.cttcanada.org/resource/resmgr/2020_conference/ abstracts/17.PNG)]. A survey was used to evaluate learning outcomes post-workshop. At Pride stem-cell drives, recruiters canvassed Pride attendees and guided eligible and interested attendees to register as donors with the Canadian Blood Services Stem Cell Registry. Following completion of each drive, recruiters recorded event outcomes and their experiences in post-event reports.

Results In 2018 and 2019, recruiters from Stem Cell Club ran 7 drives at 5 Pride festivals in 4 cities. Fourteen club leaders completed the training workshop, with 8 completing the post-workshop survey. Survey participants unanimously agreed or strongly agreed that the workshop improved their knowledge, preparation, and skills to run drives targeting recruitment of MSM. Overall, these drives recruited 354 stem-cell donors (141 men; 42\% self-reported as non-white). Recruiters from all drives shared their perspectives that efforts to engage MSM as stem cell donors were well-received by the community. They reported registering many gay, bisexual, and Trans* individuals and couples. At one drive, two LGBTQ+ people presenting in drag provided consent for their photos to be taken and published online to support efforts to engage MSM as stem-cell donors [Figure 2 (https://www. cttcanada.org/resource/resmgr/2020_conference/abstracts/17.PNG)].

Conclusions We outline a strategy to engage the MSM community as potential stem cell donors. Our work is relevant to donor recruitment organizations, donor registries, and transplant centres who seek to engage this donor population. Based on the strong outcomes to date, Stem Cell Club will spearhead drives at Pride events across Canada in 2020 to engage MSM as stem cell donors.

Affiliations: *Stem Cell Club; ${ }^{\dagger}$ University of Toronto, Faulty of Medicine, and ${ }^{\ddagger}$ University of Toronto, Faculty of Arts and Science, Toronto, ON; ${ }^{\S}$ Western University, Faculty of Medicine, London, ON; "University of Saskatchewan College of Medicine, Regina site, and "University of Regina, Faculty of Science, Regina, SK; **University of British Columbia, Faculty of Science, and ${ }^{\dagger \dagger}$ University of British Columbia, Faculty of Medicine, Vancouver, BC.

\section{Why we swab: evaluation of a library of stories in stem-cell donation in Canada four months post-launch}

Gabriele Jagelaviciute, ${ }^{* \dagger}$ Elena Kum, ${ }^{* \dagger}$ Kenneth Williams, ${ }^{* \ddagger}$ Edward W. Li, ${ }^{* \ddagger}$ Nouran Sakr, ${ }^{*}$ Santhosh Thyagu, ${ }^{\ddagger}$ Warren Fingrut*\|

Introduction Most patients in need of allogeneic stem-cell transplantation lack a matching family donor and require an alternative donor, such as an unrelated donor. Needs assessment surveys with stakeholders in stem-cell donation in Canada (newly registered stem cell donors, donor recruiters, and patients and family members) identified the need for a library of 
stories in stem-cell donation to support the education and recruitment of unrelated donors. Here, we describe an update and evaluation of Why We Swab 4 months post-launch.

Methods Why We Swab is an initiative that reports stories about Canadian stem-cell donors and recipients, patients searching for a match, and family members. The stories are directed at an audience of potential stem-cell donors, told in first-person narrative, and published alongside the storyteller's photo or video. Stories are published online to socia media (Facebook, Instagram, Twitter; @WhyWeSwab), where they are easily accessible and shareable. Prospective storytellers are invited to connect with our initiative via http://whyweswab.ca/storysubmission or by e-mail (contact@whyweswab.ca).Social media impact was evaluated using Facebook Insights. Stories from Why We Swab were included in a press release on the national Get Swabbed campaign in November 2019 , spearheaded by the Canadian donor recruitment organization Stem Cell Club. An online survey was conducted in January 2020 to determine stakeholder perspectives of Why We Swab.

Results Why We Swab launched on 21 September 2019. In the first 4 months following the launch, 12 story arcs including 21 storytellers were published. Story arcs range from 1-13 segments in length, and each segment ranges from 100 to 300 words. Ten storytellers were patients or stem-cell recipients, 5 were family members or caregivers, and 6 were stem-cell donors [Figure 1(A,B) (https://www.cttcanada.org/resource/ resmgr/2020_conference/abstracts/43-1.png)]. Several story arcs weave together accounts from a recipient and their donor, highlighting their connection in an emotional plane [Figure 2(A-C) (https://www.cttcanada. org/resource/resmgr/2020_conference/abstracts/43-2.png)]. Storytellers are from across Canada and of varying ages and ethnicities.

Altogether, story segments that were published to the Why We Swab Facebook page reached an audience of 137,590 people and secured 23,810 engagements (that is, likes, comments, and shares). Stories reported by Why We Swab were republished by 5 Canadian media outlets covering the November 2019 Get Swabbed campaign.

Fifty-one stakeholders (40 donor recruiters, 3 stem-cell recipients, 4 stem-cell donors, 4 donor registry staff) completed a survey evaluating Why We Swab. The majority felt Why We Swab helps raise awareness about stem cell donation (96\%) and supports the education of potential donors $(96 \%)$ and efforts to recruit the most-needed donors (80\%).

Conclusions We report an update on Why We Swab 4 months post-launch. In a short timeframe, stories from Why We Swab have been disseminated to a wide audience across social and traditional media. Stakeholders in donor recruitment report that the library supports donor recruitment efforts in Canada.

Affiliations: *Stem Cell Club; ${ }^{\dagger}$ Faculty of Science, Western University, London, $\mathrm{ON}$; ₹Faculty of Medicine, University of Toronto, Toronto, ON; ` Faculty of Science University of Toronto, Toronto, ON; IFaculty of Medicine, University of British Columbia, Vancouver, BC.

\section{Risk factors for graft failure in allogeneic hematopoietic cell} transplantation-a single-centre study

Zeyad Al-Shaibani, ${ }^{*}$ Eshrak Al-Shaibani,* Mats Remberger, ${ }^{+}$Wilson Lam,* Arjun D. Law,* Fotios V. Michelis, ${ }^{*}$ Auro Viswabandya,* Dennis D. Kim,* Jeffrey H. Lipton,* Jonas Mattson,* Rajat Kumar*

Introduction Allogeneic hematopoietic cell transplantation (allo-HCT) is a curative therapy for high-risk hematologic diseases and sustained engraftment of the donor stem cells is essential for transplant success. Graft failure (GF) is a serious complication post allo-HCT. In the presented study we aimed to assess the incidence, risk factors in a single-centre population, and the impact on transplant outcome.

Methods Retrospective data were collected between January 2015 and December 2018 and updated in June 2019. A total of 557 patients transplanted. Primary GF was defined as failure to achieve an absolute neutrophil count (ANC) $>500 / \mu \mathrm{L}$ by 28 days after bone marrow or peripheral blood stem cell transplantation. In contrast, secondary GF was defined as cytopenias after initial engraftment (ANC $<500 / \mu \mathrm{L}$ ), with donor chimerism of $<5 \%$ or falling donor chimerism with intervention such as second transplant or donor lymphocyte infusion (DLI) or patient death due to cytopenias, and fall in chimerism, even if chimerism $>5 \%$. Exclusion criteria were disease relapse, graft-versus-host disease or other causes of cytopenias such as, viral infections, or drug induced. Outcomes examined included overall survival (os), cumulative incidence of GF, non-relapse mortality (NRM), and cause of death Results GF was seen in $43(7.7 \%)$ patients. Of these 43 patients, $9(21 \%)$ had primary GF, and 34 (79\%) had secondary GF. The cumulative incidence of GF overall (primary and secondary) is $1.6 \%(0.8 \%-3.0 \%)$ at day 100 and $6.5 \%$ $(4.5 \%-8.8 \%)$ at day 800 . The median survival of patients after primary GF was 41 days versus 144 days in secondary GF. At 100 days, os was $22 \%$ in primary GF and $64 \%$ in secondary GF. The 1-year and 2 -year os for secondary
GF was $33 \%$ and $28 \%$ respectively. Multivariable analysis demonstrated that the transplant indication myelodysplastic syndrome, myelofibrosis, lymphoma, or nonmalignant diseases) and donor type (HLA-mismatched unrelated or haploidentical) were the only factors significantly associated with increased GF.

We determined the effect of more than one of these risk factors on the occurrence of GF as seen in in Figure 1 (https://www.cttcanada.org/ resource/resmgr/2020_conference/abstracts/18.png).

In primary GF, 5 patients underwent second allo-HCT vs. 15 patients (44\%) from secondary GF, and another 8 patients received DLI. All the patients with primary GF died because of GF and its associated complications. In secondary GF, 22 patients (51\%) died, $30 \%$ of causes related to infections. Conclusions Our study showed an increased risk for GF following the use of mismatched unrelated or haploidentical donors for diseases such as lymphoma, myelofibrosis, myelodysplastic syndrome, and nonmalignant diseases. As well, we found that a presence of 2 risk factors puts patients at significant increased risk of GF. More intense conditioning therapy should be considered for patients with 1 but in particular 2 risk factors.

Affiliations: *Hans Messner Allogeneic Transplant Program, Princess Margare Cancer Centre, University Health Network, University of Toronto, Toronto, ON; ${ }^{\dagger}$ KFUE, Uppsala University Hospital and Institution of Medical Science, Uppsala University, Uppsala, Sweden.

\section{Comparison of the prognostic ability of the HCT-CI, the modified EBMT, and the EBMT-ADT scores for patients with acute myeloid leukemia undergoing allogeneic stem-cell transplantation} Eshrak Al-Shaibani,* Sunu Cyriac, ${ }^{*}$ Shiyi Chen, ${ }^{+}$Jeffrey H. Lipton, ${ }^{*}$ Dennis D. Kim,* Auro Viswabandya,* Rajat Kumar,* Wilson Lam,* Arjun Law,* Zeyad Al-Shaibani,* Armin Gerbitz, ${ }^{*}$ Ivan Pasic,* Jonas Mattsson,* Fotios V. Michelis*

Background Allogeneic hematopoietic cell transplantation (НСT) may provide cure for acute leukemia where indicated. However, it is associated with significant morbidity and mortality. This has prompted the development of pre-transplant risk scores such as the Hematopoietic Cell Transplantation-Comorbidity Index (HCT-CI) and the modified European Group for Blood and Marrow Transplantation (mEBMT) score. In a large EBMT study (Shouval et al., JCO 2015) developed a machine learning-based prediction model for mortality (EBMT-ADT) that can be used online.

Methods We retrospectively examined the impact of the HCT-CI, mEBMT, and EBMT-ADT on 231 patients who underwent allogeneic HCT for acute leukemia from August 2014 to December 2017. Data were updated as of October 2019. Results Median age 56 years (range: 19-72). Acute myeloid leukemia (AML) diagnosed in 200, acute lymphoblastic leukemia (ALL) in 31. Disease status at the time of HCT was first complete remission (CR1) in $81 \%$ and CR2 or beyond in $19 \%$. Myeloablative conditioning was administered to $31 \%$, while $69 \%$ received reduced-intensity conditioning. In vivo $\mathrm{T}$ cell depletion was given to $76 \%$ of patients. Donors were HLA matched related in $33 \%, 10 / 10$ matched unrelated in $44 \%, 9 / 10$ unrelated in $12 \%$, and haploidentical in $11 \%$ of patients. The median follow-up of survivors was 37 months (range: 15-62).

For the HCT-CI, patients were grouped as score $0-1,2-3$, and $>3(116,75$, and 40 patients respectively). For the mEBMT score, patients were grouped as $0-2,3$, and $4-5(76,121$, and 34 patients respectively), while for the EBMT-ADT, the respective 100-day mortality was calculated for each patient (excluding haploidentical) and grouped as $\leq 4.1 \%, 4.1 \%-11.5 \%$, and $>11.5 \%$. Patients with higher HCI-CI score demonstrated lower probability for os ( $p=0.038$; C-statistic: 0.57 ), while neither mEBMT nor EBMT-ADT significantly stratified patients into prognostic groups ( $p=0.09$; C-statistic: 0.54 ; and $p=0.28$; C-statistic: 0.53 respectively). Multivariable analysis for os demonstrated that leukemia type (HR for ALL: $1.82 ; 95 \%$ CI: 1.16 to $2.85 ; \mathrm{p}=0.01$ ), HLA matching (HR for ALL fully matched donors: $0.61 ; 95 \% \mathrm{CI}: 0.41$ to $0.90 ; p=0.01$ ) and HCT-CI (HR for $0-1: 0.50 ; 95 \%$ CI: 0.31 to $0.81 ; p=0.004$ ) had significant impact. Based on the HR, a new weighted score was developed for this cohort including leukemia type $(\mathrm{AML}=0, \mathrm{ALL}=1)$, HLA matching (fully matched $=0$, mismatch unrelated or haploidentical $=1)$ and HCT-CI $(0-1=0,2-3=1$, and $\geq 4=2)$. The new score demonstrated improved prognostic capability compared with the other scores [ $p=0.0004$; C-statistic: 0.60; Figure (https://www.cttcanada. org/resource/resmgr/2020_conference/abstracts/19.PNG)].

Conclusions The mEBMT score and the EBMT-ADT do not adequately prognosticate os in our cohort of acute leukemia patients undergoing allogeneic НСт. The HCT-CI performs better, but is not a powerful predictor. New approaches are required to develop a pre-transplant risk calculation tool that can be widely applicable to patients from various centres characterized by heterogeneous practices.

Affiliations: *Hans Messner Allogeneic Transplant Program and ${ }^{\dagger}$ Department of Biostatistics, Princess Margaret Cancer Centre, Toronto, ON. 


\section{Outcome of second allogeneic stem-cell transplantation versus donor lymphocyte infusion following allogeneic stem-cell transplantation-a single-centre study}

Eshrak Al-Shaibani, Jeffrey H. Lipton, Dennis D. Kim, Auro Viswabandya, Rajat Kumar, Wilson Lam, Arjun Law, Zeyad Al-Shaibani, Armin Gerbitz, Ivan Pasic, Jonas Mattsson, Fotios V. Michelis

Background Allogeneic hematopoietic cell transplantation (HCT) is potentially curative for a variety of hematologic diseases, but may be complicated by disease relapse or graft loss/poor graft function. Second allogeneic HCT and donor lymphocyte infusions (DLI) are utilized in the management of both complications. The purpose of the present study is to compare outcomes following both interventions in a single-centre cohort of patients transplanted over the last 5 years.

Methods We retrospectively investigated 65 patients, 34 (52\%) underwent second allogeneic HCT and 31 (48\%) received DLI, from June 2011 to November 2018.

Results Median age at the time of the post-transplant intervention was 51 years (range: 19-72). Second HCT was performed for disease relapse in 11 patients (5 of whom were transplanted for AML) and for graft failure (GF) in 23 patients (primary in 6 and secondary in 17 patients). Donors for second HCT were related $(n=10)$, unrelated $(n=18)$, and haploidentical $(n=6)$. The same donor was used in $20(59 \%)$ patients. At second HCT, the HCT-CI was $0-2$ for 22 patients $(65 \%)$ and $\geq 3$ for 12 patients (35\%). The DLI was performed for relapse for 17 patients ( 8 of whom were transplanted for AML) and for secondary GF or chimerism loss in 14 patients. Donors for DL were related for 13 patients, unrelated for 16 patients, and haploidentical for 2 patients. Karnofsky performance status (KPS) at first DLI was $90 \%-100 \%$ for 20 patients $(65 \%)$ and $70 \%-80 \%$ for 11 patients (35\%). Median number of DLI administered was 2 (1-11).

Median follow-up of survivors following intervention was 18 months (range: 3-66 months). Univariate analysis demonstrated 2-year overall survival (OS) of $50 \%$ (95\% CI: $37 \%$ to $62 \%$ ) for the entire cohort, while 2 -year os was identical between all second transplant patients and DLI patients (50\% for both groups, $p=0.9 \%$ ). For second HCT patients, 2 -year os was $82 \%$ (95\% CI: $45 \%$ to $95 \%$ ) and $35 \%$ (95\% CI: $17 \%$ to $54 \%$ ) for relapse as an indication versus GF respectively $(p=0.01)$. For the DLI patients, 2 -year os was $44 \%$ (95\% CI: $20 \%$ to $66 \%$ ) and $56 \%$ (95\% CI: $27 \%$ to $78 \%$ ) for relapse as an indication versus GF/ loss of chimerism respectively $(p=0.64)$. For disease relapse as an indication, second HCT was significantly superior to DLI $[p=0.03$; Figure (https://www. cttcanada.org/resource/resmgr/2020_conference/abstracts/20.PNG)] Multivariable analysis for second НCT patients demonstrated age (HR: 1.04 95\% CI: 1.00 to $1.07 ; p=0.03$ ) and donor type (HR for related donor: $0.17 ; 95 \%$ CI: 0.04 to $0.75 ; p=0.02$ ) to independently predict OS. For DLI, multivariable analysis demonstrates KPS at the time of the first dose of DLI as the predominant predictor of survival (HR: 0.89 ; 95\% CI: 0.82 to 0.96 ; $p=0.002$ ).

Conclusions Second allogeneic НCT demonstrates superior survival when performed for disease relapse post-transplant compared with DLI. In contrast, second HCT for graft failure results in poor long-term survival. Second allogeneic НCT should be preferred as a reasonable treatment option for patients that relapse post-transplant and achieve pre-second HCT remission

Affiliation: Hans Messner Allogeneic Transplant Program, Princess Margaret Cancer Centre, Toronto, ON.

\section{High rates of allogeneic transplantation and successful} engraftment in older patients with active, relapsed, or refractory (rel/ref) AML following targeted conditioning with anti-CD45 iodine $\left({ }^{131} \mathrm{I}\right)$ apamistamab [lomab-B]: preliminary midpoint results from the prospective, randomized phase 3 SIERRA trial

Boglarka Gyurkocza,* Rajneesh Nath, Patrick J. Stiff, Edward Agura, Mark R. Litzow, Benjamin Tomlinson, Hannah Choe, Sunil Abhyankar, Stuart E. Seropian, George L. Chen, Parameswaran Hari, Zaid AlKadhimi, James Foran, Johnnie J. Orozco, Koen van Besien, Mitchell Sabloff, ${ }^{\dagger}$ Partow Kebriaei, Camille Abboud, Moshe Yair Levy, Hillard M. Lazarus, Sergio A. Giralt, Mark S. Berger, Vijay Reddy, John M. Pagel

Background Patients with rel/ref AML have very few options, despite novel targeted therapies (for example, venetoclax, IDH inhibitors), other than allogeneic hematopoietic cell transplantation (НСT) to achieve a durable CR. In addition, patients $\geq 55$ years of age cannot tolerate multiple cycles of intensive therapy trying to achieve a CR prior to transplant. Older patients are also unable to tolerate more intense myeloablative conditioning. The SIERRA trial is a prospective, randomized, phase 3 trial addressing the unmet need to improve access of older patients with active, rel/ref AML to HCT, the only potentially curative therapy available to them.

Methods Patients are randomized (1:1) to receive Iomab-B (day -12) followed by FLU/TBI and HCT, or to conventional care (CC). Patients in CC may receive investigator's choice of salvage therapy, including newly approved targeted agents and may proceed to standard HCT if they achieve CR. If patients do not achieve CR, the study allows cross-over (CO) to Iomab-B/HCT. Results Preliminary data are available from $75(50 \%)$ patients [Table 1 (https://www.cttcanada.org/resource/resmgr/2020_conference/ abstracts/21.PNG)]. Patients (median age 64) were heavily pretreated with $85 \%$ failing $\geq 2$ induction therapies and $33 \%$ failing targeted therapies. All patients were transplanted after Iomab-B engrafted. After randomization, $82 \%(31 / 38)$ patients in the CC arm failed salvage therapy, despite $32 \%$ $(12 / 38)$ receiving targeted therapy. Of the patients receiving venetoclax with HMA or LDAC, $73 \%(8 / 11)$ did not achieve remission. Of the 31 patients, 9 were not co transplant candidates. Of the 22 patients that crossed over for transplant, a majority $(91 \%, n=20)$ were able to receive Iomab-B/HCT, despite a median of $35 \%$ BM blasts. The most common reason preventing co to transplant was disease progression. Patients in CC showed increased incidence of grade 3 febrile neutropenia, compared with Iomab-B patients prior to CO or HCT ( $34 \%$ vs. $8 \%$ ). Iomab-B administration was generally well-tolerated with 1 grade 3 and no grade 4 , infusion reaction. There were no Iomab-B-related deaths and 100-day non-relapse TRM was $3 \%$ on the Iomab-B arm [Table 1 (https://www.cttcanada.org/resource/ resmgr/2020_conference/abstracts/21.PNG[Table 1 (

Conclusions Despite advanced age, active disease, and heavily pretreated patients, $100 \%(31 / 31)$ of all patients in the Iomab-B arm who received treatment with Iomab-B were able to undergo НCT and successfully engrafted. In comparison, only $18 \%(7 / 38)$ of the patients on the CC arm achieved remission and underwent conventional HCT despite a high percentage receiving recently approved agents and targeted therapies. Overall, including patients crossing over to receive Iomab-B/HCT after failure of salvage therapy in the CC arm, $68 \%(51 / 75)$ of all patients enrolled in the trial underwent HCT after Iomab-B. These preliminary results represent significant improvements in the current rates of transplantation in this patient population. This SIERRA trial is currently enrolling (https://www. sierratrial.com/ or NCT02665065 at https://ClinicalTrials.gov/).

Affiliations: *Memorial Sloan Kettering Cancer Center, New York, NY, U.S.A.; †The Ottawa Hospital, University of Ottawa, Ottawa, ON.

\section{Outcome of allogeneic stem-cell transplantation in elderly} patients: a single-centre study

Eshrak Al-Shaibani,* Shiyi Chen, ${ }^{\dagger}$ Zeyad Al-Shaibani,* Wilson Lam,* Arjun D. Law,* Ivan Pasic,* Fotios V. Michelis,* Auro Viswabandya,* Dennis D. Kim,* Armin Gerbitz,* Jeffrey H. Lipton,* Jonas Mattson,* Rajat Kumar*

Background Allogeneic hematopoietic stem-cell transplantation (allo-HCT) is being offered to older patients. Data about their outcomes and period of hospitalization are limited. We aimed to study these factors. Methods We retrospectively analyzed the outcome in 259 consecutive patients aged $\geq 60$ years who underwent allo-HCT, from January 2014 to January 2019. Diagnosis was AML in $49 \%$, MDS in $20 \%$, MF in $15 \%$, ALL in $6 \%$, and $10 \%$ for the rest. Conditioning regimen was mainly reduced-intensity (Flu Bu TBI 200) in 94\%; in vivo T cell-depleted was given in $85 \%$ of patients. Data were updated on January 2020. We categorized patients into 3 age groups $\geq 60$ to $65,>65$ to 70 , and $>70$. Outcomes examined included overall survival (OS), progression-free survival (PFS), non-relapse mortality (NRM), length of hospitalization for transplant, the number and reasons of re-hospitalization during the first year of НCT.

Results The median age was 65 years (range: $60-74$ ), and $60 \%$ were men. Karnofsky performance status (KPS) was $\geq 80$ in $94 \%$ of patients. The median follow-up up was 13 months (range: 1-69). Donors were HLA matched related in $27 \%, 10 / 10$ matched unrelated in $47 \%, 9 / 10$ unrelated in $13 \%$, and haploidentical in $13 \%$ of patients. A majority of patients (98\%) received PBSC graft. The median days of hospitalization during the transplant period was 31 days (range: $20-132$ ) and was not statistically different in different age groups ( $p=0.22$ ). The re-hospitalization within the first 6 months was $68 \%$; in the second 6 months, it was $39 \%$ and was not statistically significant $(p=0.20,0.96)$. The main cause of re-hospitalization was infection; $46 \%$ in first 6 months and $23 \%$ in the second 6 months. The os at 1 year was lower in age $>70$ years $(32 \%)$ vs. $(56 \%)$ in $>65$ to $\leq 70$, and $53 \%$ for age $\geq 60$ to $\leq 65$ $(p=0.03)$. The NRM at 1 year was inferior in age group $>70(56 \%)$ vs. (35\%) in $>65$ to $\leq 70$ and $26 \%$ for age $\geq 60$ to $\leq 65$ [p $=0.0029$; Table 1 (https: / www cttcanada.org/resource/resmgr/2020_conference/abstracts/22.PNG)].

Conclusions The outcomes of OS, PFS, and NRM are inferior in age $>70$, and the hospitalization duration and re-hospitalization rates are not different in various age groups.

Affiliations: *Hans Messner Allogeneic Transplant Program, Princess Margare Cancer Centre, University Health Network, University of Toronto, and ${ }^{\dagger}$ Department of Biostatistics, Princess Margaret Cancer Centre, Toronto, ON. 


\section{Building a community of practice to improve stem-cell donor recruitment in Canada}

Elena Kum, ${ }^{*+}$ Adriyan Hrycyshyn, ${ }^{*+}$ Gabriele Jagelaviciute, ${ }^{*+}$ Angela C. Chen, ${ }^{* \neq}$ Iman Baharmand, ${ }^{*}$ Samer Rihani, ${ }^{*}$ Gabriella Rumball, ${ }^{* \|}$ Rana Kandel, ${ }^{* *}$ Sylvia Okonofua, ${ }^{* * *}$ Edward Wei Li, ${ }^{*++}$ Sze Wah Samuel Chan, ${ }^{*++}$ Shamini Vijaya Kumar, ${ }^{*++}$ Kenneth Williams, ${ }^{*++}$ Daniel Tarade, ${ }^{*++}$ Lillie Prokosch, ${ }^{* \neq \ddagger}$ Warren Fingrut $* \$ \S$

Background A community of practice (COP) is a group of people who share a passion for something and learn how to perform better as they interact regularly. These groups have been shown to be effective models for achieving quality outcomes in health care.

Purpose We report the development and evaluation of a COP in stem-celldonor recruitment in Canada, with the goal of supporting recruitment of the most needed stem-cell donors.

Methods In September 2017, we launched a COP in stem-cell donor recruitment in Canada. Stakeholders in donor recruitment were invited via e-mail and Facebook posts to participate in regular e-meetings and a Facebook group. E-meeting topics included running larger stem-cell drives, recruiting the most needed donors, redirecting non-optimal donors, reviews of drive outcomes and strategies to improve, using patient stories to support donor recruitment, and reducing donor attrition. Each e-meeting included speakers and roundtable discussion relevant to the theme. The Facebook group facilitated discussion and sharing of resources between e-meetings. A survey was sent to COP participants in January 2020 to evaluate the perceived impact of the COP on donor recruitment practice. Recruitment outcomes by COP participants of the Canadian donor recruitment organization Stem Cell Club were compared before and after the launch of the cop.

Results As of January 2020, the COP Facebook group included 281 stakeholders in donor recruitment: 266 donor recruiters from Stem Cell Club, 7 patients, and 6 donor registry staff. Fifty-one unique attendees participated in 7 e-meetings (median attendees per meeting: 14; range: 11-19). In total, 141 posts were published to the Facebook group about patient/donor stories (41\%), resources in stem-cell donation (23\%),stem-cell drive outcomes and campaigns (15\%), updates related to donor recruitment (14\%), and questions posed to the community by cop participants (5\%).

Forty-four COP participants completed the COP evaluation survey. The majority agreed or strongly agreed that the Facebook group (86\%) and e-meetings (59\%) supported the development of a community; $64 \%-84 \%$ agreed or strongly agreed that participating in the cop fostered collaboration, improved their knowledge and practice in donor recruitment, and improved their ability to run higher quality drives and recruit most-needed donors [Figure 1 (https://www.cttcanada.org/resource/resmgr/2020_conference/ abstracts/23.PNG)]. Stem Cell Club's donor recruitment outcomes improved following the launch of the COP: in 2016-2017, Stem Cell Club recruited 2918 donors ( $46 \%$ men; $55.9 \%$ of men non-white) compared with 3418 donors in 2017-2018 (52.7\% men; $57.8 \%$ of men non-white), and 4531 donors in 2018-2019 [52.9\% men; 62.7\% of men non-white; Figure 2 (https://www. cttcanada.org/resource/resmgr/2020_conference/abstracts/24.png).

Conclusions We describe the first COP in stem-cell donor recruitment in the world to our knowledge. The COP was valued by participants and supported efforts to improve donor recruitment. The cop model could be adapted by other donor recruitment organizations around the world to improve recruitment outcomes.

Affiliations: *Stem Cell Club; ${ }^{\dagger}$ Faculty of Science, Western University, London, ON; ${ }^{\ddagger}$ Faculty of Science, University of Waterloo, Waterloo, ON; ${ }^{\circledR}$ Faculty of Science, Simon Fraser University, Burnaby, BC; "Faculty of Science, Laurentian University, Sudbury, ON; "Faculty of Medicine, University of Ottawa, Ottawa, ON; **Faculty of Science, University of Regina, Regina, SK; ${ }^{+\dagger}$ Faculty of Medicine, University of Toronto, Toronto, ON; 抽Faculty of Science, Wilfrid Laurier University, Waterloo, ON; ${ }^{\S} \mathrm{F}$ Faculty of Medicine, University of British Columbia, Vancouver, BC.

\section{Correlation of bridging and lymphodepleting chemotherapy with clinical outcomes in patients with relapsed/refractory diffuse large B cell lymphoma treated with tisagenlecleucel}

Charalambos Andreadis, ${ }^{*}$ Constantine S. Tam, ${ }^{\dagger}$ Peter Borchmann, ${ }^{\ddagger}$ Ulrich Jäger, ${ }^{\S}$ Joseph P. McGuirk, , Harald Holte, ${ }^{\sharp}$ Edmund K. Waller, ${ }^{* *}$ Samantha Jaglowski, ${ }^{+\dagger}$ Michael R. Bishop, ${ }^{\ddagger \ddagger}$ Stephen Ronan Foley,,$\$$ Jason R. Westin, IIIII Isabelle Fleury,,"\# P. Joy Ho, ${ }^{* * *}$ Stephan Mielke, ${ }^{++}$ Takanori Teshima, ${ }^{\neq \neq \ddagger}$ Gilles Salles, $\$$ $\$$ Stephen J. Schuster, IIIIII Veronika Bachanova, ${ }^{\# \#}$ Richard T. Maziarz, ${ }^{* * * *}$ Koen Van Besien, ${ }^{+++t}$ Koji Izutsu, ${ }^{\neq \neq \neq \neq}$John M. Magenau, ${ }^{\S \S \S \S}$ Nina Wagner-Johnston, ||||||||| Koji Kato, ${ }^{\sharp \# \# ~ P a o l o ~ C o r r a d i n i, ~}{ }^{* * * * *}$ Ranjan Tiwari, ${ }^{+++++}$Rakesh Awasthi, ${ }^{, \neq \neq \neq \neq}$

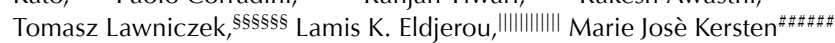

Background Tisagenlecleucel is an autologous anti-CD19 chimeric antigen receptor (CAR)-T cell therapy approved for adult patients (pts) with relapsed/refractory diffuse large $B$ cell lymphoma ( $\mathrm{R} / \mathrm{R}$ DLBCL). In the pivotal, phase 2 JULIET trial (NCT02445248), pts could receive bridging therapy (вт) to allow flexibility in scheduling and maintain disease control. Lymphodepleting chemotherapy (LDC) was started 5-14 days prior to CAR-T cell infusion. Here we present baseline characteristics, clinical outcomes, and cellular kinetics by вт and type of LDC used in the JULIET trial. Methods Patients were categorized based on BT or no BT, as well as LDC [cyclophosphamide/fludarabine (Cy/Flu) or bendamustine (previous grade 4 hemorrhagic cystitis with Cy or demonstrated resistance to a previous Cy-containing regimen)]. Lymphodepleting chemotherapy was not required if white blood cell count was $<1000$ cells $/ \mu \mathrm{L}$ within 1 week prior to infusion. Best overall response rate (ORR) was defined as complete response (CR) + partial response (PR). Time course of transgene copies per microgram of genomic DNA, as measured by qPCR, was used to estimate cellular kinetic parameters (maximal expansion in vivo, $C_{\max }$; time to maximal expansion, $T_{\max }$; area under the curve from time of infusion to day 28, AUC0-28d).

Results Of the $115 \mathrm{pts}$ infused, 104 received вт and $11 \mathrm{pts}$ did not receive вт. For LDC, 85 pts received Cy/Flu, 22 bendamustine, and 8 pts did not receive LDC. Baseline and disease characteristics across categories are presented [Table (https://www.cttcanada.org/resource/resmgr/2020_conference/ abstracts/25.png)].

Patients who did not receive BT had an ORR of $81.8 \%$ (63.6\% CR); in pts who received BT, the ORR was $49.0 \%(35.6 \% \mathrm{CR})$ [Table (https://www. cttcanada.org/resource/resmgr/2020_conference/abstracts/25.png)]. Progression-free survival (PFS) rate at 12 months was $60.6 \%$ in pts who did not receive вт and $32.0 \%$ in pts who received вт. Among LDC groups, pts who received Cy/Flu had an ORR of $57.6 \%$ (41.2\% CR) and 12-month PFS rate of $39.1 \%$, whereas those who received bendamustine had an ORR of $40.9 \%(31.8 \% \mathrm{CR})$ and 12 -month PFS rate of $21.2 \%$. These differences were not statistically significant.

Rates of selected adverse events reported $\leq 8$ weeks post-infusion, $>8$ weeks to $\leq 1$ year post-infusion, $>1$ year post-infusion, and any time after infusion were generally consistent across BT and LDC groups for prolonged cytopenias, neurologic events (NE), cytokine release syndrome (CRS), and infection [Table (https://www.cttcanada.org/resource/resmgr/2020_conference/abstracts/25.png)].

$C_{\max }, T_{\max }$, and exposure ( $\mathrm{AUC}_{0-28 \mathrm{~d}}$ ) were similar between LDC groups [Table (https://www.cttcanada.org/resource/resmgr/2020_conference/ abstracts/25.png)]. $C_{\max }$ and $\mathrm{AUC}_{0-28 \mathrm{~d}}$ were also similar between pts who did and did not receive BT.

Conclusions A majority of pts in the JULIET trial received BT and LDC, indicating high tumour burden and aggressive disease in this pt population with R/R DLBCL. Although sample size is small $(n=11)$, pts not requiring BT appeared to have less-aggressive disease, achieved high response rates, and had no grade 4 CRS or NE. Further evaluation of the impact of BT and LDC on clinical outcomes on a larger patient population will be possible with the availability of registry data.

Affiliations: *Department of Medicine, University of California San Francisco, Helen Diller Family Comprehensive Cancer Center, San Francisco, CA, U.S.A.; ${ }^{\dagger}$ Peter MacCallum Cancer Centre, St. Vincent Hospital and University of Melbourne, Melbourne, VIC, Australia; ${ }^{\ddagger}$ German Hodgkin Study Group and Department of Medicine I, Center for Integrated Oncology Aachen Bonn Cologne Düsseldorf, University Hospital Cologne, Cologne, Germany; ${ }^{\S}$ Clinical Division of Hematology and Hemastaseology, Department of Medicine I, Vienna General Hospital-Medical University of Vienna, Vienna, Austria;"Department of Internal Medicine, The University of Kansas Health System, Kansas City, KS, U.S.A.; "Department of Oncology, Oslo University Hospital, Oslo, Norway; **Winship Cancer Institute, Emory University, Atlanta, GA, U.S.A.; †The Ohio State University, Columbus, OH, U.S.A.; 非Hematopoietic Cellular Therapy Program, Section of Hematology/Oncology, University of Chicago, Chicago, IL, U.S.A.; \$\$ Hamilton Health Sciences, Hamilton, ON; IIIIDepartment of Lymphoma and Myeloma,

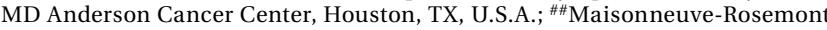
Hospital, University of Montreal, Montreal, QC; ***Department of Haematology, Institute of Haematology, Royal Prince Alfred Hospital, Camperdown, NSW, Australia; ${ }^{\dagger \dagger}$ University Hospital Würzburg, Center of Allogeneic Stem Cell Transplantation, Department of Internal Medicine II, Würzburg University Medical Center, Würzburg, Germany; 㧊Department of Hematology, Hokkaido University Hospital, Hokkaido, Japan; ${ }^{\$ \$ \$}$ Department of Pathology and Laboratory Medicine, Hospices Civils de Lyon, Lyon-Sud Hospital, Pierre-Bénite, France IIIIILymphoma Program, Abramson Cancer Center, University of Pennsylvania, Philadelphia, PA, U.S.A.; "\#\#\#ivision of Hematology, Oncology and Transplantation, University of Minnesota, Minneapolis, MN, U.S.A.; **** Oregon Health and Science University, Portland, OR, U.S.A.; ${ }^{\dagger+\dagger}$ Division of Hematology and Oncology, Weill Cornell Medicine, New York, NY, U.S.A.; ${ }^{\ddagger \neq \ddagger \neq}$ Department of Hematology, National Cancer Center Hospital, Tokyo, Japan; ${ }^{\$ \$ \$ s}$ Blood and Marrow Transplantation Program, University of Michigan, Ann Arbor, MI, U.S.A.; IIIIIIISSidney Kimmel Comprehensive Cancer Center, Johns Hopkins School of Medicine, Baltimore, MD, U.S.A.; "\#\#\#\# Department of Medicine and Biosystemic 
Science, Kyushu University Hospital, Fukuoka, Japan; ${ }^{* * * * * F o n d a z i o n e ~ I R C C S ~}$

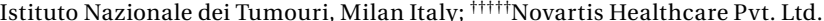
Hyderabad, India; ${ }^{\ddagger \ddagger \ddagger \neq \ddagger}$ Novartis Institutes for BioMedical Research, East Ha-

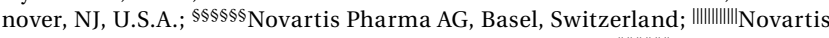
Pharmaceuticals Corporation, East Hanover, NJ, U.S.A.; \#\#\#\#\#\#Department of Hematology, Academic Medical Center, Amsterdam, Netherlands.

\section{Correlative analyses of patient and clinical characteristics associated with efficacy in tisagenlecleucel-treated relapsed/ refractory diffuse large B cell lymphoma patients in the JULIET trial} Jason R. Westin, ${ }^{*}$ Constantine S. Tam, ${ }^{\dagger}$ Peter Borchmann, ${ }^{\ddagger}$ Ulrich Jaeger, $\$$ Joseph P. McGuirk, I Harald Holte, ${ }^{\#}$ Edmund K. Waller,** Samantha Jaglowski, ${ }^{+\dagger}$ Michael R. Bishop, ${ }^{\neq \ddagger}$ Charalambos Andreadis, ${ }^{\S \S}$ Stephen Ronan Foley, IIII Isabelle Fleury, ${ }^{\#}$ P. Joy Ho, ${ }^{* * *}$ Stephan Mielke, ${ }^{++\dagger \neq \neq}$ Takanori Teshima, $\$ \S \S$ Stephen J. Schuster,,|||||| Veronika Bachanova, ${ }^{\# \#}$ Richard T. Maziarz, ${ }^{* * * *}$ Koen van Besien, ${ }^{+++\dagger}$ Koji Izutsu, ${ }^{\neq \neq \neq \ddagger}$ Marie Jose Kersten, $\$ \S \S \S$ John M. Magenau, IIII|||| Nina Wagner-Johnston, \#\#\# Koji Kato, ${ }^{* * * * *}$ Paolo Corradini, ${ }^{++++}$Xia Han, ${ }^{\neq \neq \neq \neq \neq}$Ranjan Tiwari, ${ }^{\S \S \S \S}$ Sergei Agoulnik, |||||||||| Lamis K. Eldjerou, ${ }_{\neq \neq \neq \neq \neq}^{\neq i d a ~ B u b u t e i s h v i l i ~ P a c a u d, ~}$ Gilles Salles $\# \# \#$

Background Tisagenlecleucel has demonstrated durable responses and a manageable safety profile in adult patients (pts) with relapsed/refractory diffuse large B cell lymphoma (R/R DLBCL). We report the correlation of pre- and post-infusion factors and biomarkers with efficacy in pts with $\mathrm{R} / \mathrm{R}$ DLBCL treated with tisagenlecleucel.

Methods Results from JULIET (NCT02445248), a global, pivotal, phase 2 trial of tisagenlecleucel in adults with R/R DLBCL, were analyzed to identify baseline disease and pt characteristics and serum biomarkers that may correlate with efficacy. Relationships between lactate dehydrogenase (LDH), baseline tumour volume (TV, within 30 days prior to infusion), pre-treatment C-reactive protein and thrombocytopenia, as well as cytokine release syndrome (CRS; Penn scale)/neurological events (NE; CTCAE v4.03) severity, and efficacy outcomes were assessed.

Results As of 11 December 2018, 115 pts were infused with tisagenlecleucel and evaluable for efficacy. Baseline TV did not correlate with month (mo) 3 response. Median LDH at multiple pre-infusion time points was higher in nonresponders (NRs) vs. pts achieving complete/partial response (CR/ PR). Out of 16 pts, 15 with pre-infusion grade (G) 3/4 thrombocytopenia $\left(<50 \times 10^{9} / \mathrm{L}\right)$ were NRs. Uni- and multivariate regression analyses showed high pre-infusion LDH [more than twice the upper limit of normal (ULN)] was independently associated with NRs. Pts with LDH above ULN had poorer PFS and oS vs. those with normal pre-infusion LDH [Figure 1 (https://www. cttcanada.org/resource/resmgr/2020_conference/abstracts/26.png)]. Pts with pre-infusion platelet levels $<50 \times 10^{9} / \mathrm{L}$ also had significantly worse PFs and os vs. those with platelet levels $\geq 50 \times 10^{9} / \mathrm{L}$.

Post-infusion, correlations between severe (G 3/4) CRS/NE and efficacy were examined. All 13 pts with severe NE were NRs, as were $9 / 17$ pts with G 3 CRS and all 9 pts with G 4 CRS. Pts with severe CRS or NE had worse PFS and os vs. pts with grade $0-2$ CRS or NE respectively. High pre-infusion LDH and G 3/4 thrombocytopenia and severe NE were independently associated with poorer PFS in Cox regression analyses.

Additional analysis indicated that highest levels of serum biomarker, highest LDH and lowest platelet counts $\leq 1$ mo post-infusion were seen in pts with severe CRS who were also NRs, compared with pts with severe CRS who achieved CR/PR and pts with G 0-2 CRS.

Conclusions Multivariate analyses identified that high levels of preinfusion LDH was associated with NRs at mo 3 as well as worse PFS and os. Grade 3/4 thrombocytopenia at pre-infusion and G 3/4 NE were also associated with poor efficacy outcomes. The highest serum biomarker profiles post-infusion appeared to associate with pts with severe CRS who were also NRs. These analyses suggest that a subset of pts with aggressive disease at infusion and/or pts with severe CRS/NE had poorer outcomes in the JULIET trial. These analyses may reinforce the rationale for use of CAR-T cell therapy in earlier lines, optimizing pt care, and preventing severe CRS and/or NE.

Affiliations: *Department of Lymphoma and Myeloma, MD Anderson Cancer Center, Houston, TX, U.S.A.; ${ }^{\dagger}$ Peter MacCallum Cancer Centre, St. Vincen Hospital and University of Melbourne, Melbourne, Australia; ${ }^{\ddagger}$ University Hospital of Cologne, Cologne, Germany; ${ }^{\S}$ Clinical Division of Hematology and Hemastaseology, Department of Medicine I, Vienna General Hospital-Medical University of Vienna, Austria; "Department of Internal Medicine, The University of Kansas Health System, Kansas City, KS, U.S.A.; Department of Oncology, Oslo University Hospital, Oslo, Norway; **Emory Clinic, Division of Hematology/Oncology, Atlanta, GA, U.S.A.; ${ }^{\dagger \dagger}$ The Ohio State University, Columbus, OH, U.S.A.; ${ }_{\ddagger \ddagger}$ Hematopoietic Cellular Therapy Program Section of Hematology/Oncology, University of Chicago, Chicago, IL, U.S.A.; ${ }^{\S}$ Department of Medicine, University of California San Francisco, Helen Diller Family Comprehensive Cancer Center, San Francisco, CA, U.S.A.; IIIMcMaster University, Hamilton, ON, Canada
\#\#University Paris Diderot, Montreal, Canada; ***Department of Haematology, Institute of Haematology, Royal Prince Alfred Hospital, Camperdown NSW, Australia; ${ }^{\dagger \dagger}$ University Hospital Wurzbürg, Würzburg, Germany; ${ }^{\ddagger \ddagger}$ Karolinska Institutet and University Hospital, Stockholm, Sweden; ${ }^{\$ \S}{ }^{\$}$ Department of Hematology, Hokkaido University Hospital, Hokkaido, Japan; IIIIILymphoma Program, Abramson Cancer Center, University of Pennsylvania, Philadelphia, PA, U.S.A.; \#\#\#Division of Hematology, Oncology and Transplantation, University of Minnesota, Minneapolis, MN, U.S.A.; ${ }^{* * * *}$ Oregon Health and Science University, Portland, OR, U.S.A.; ${ }^{\dagger+\dagger}$ Department of Pathology and Laboratory Medicine, Weill Cornell Medicine, New York, NY, U.S.A.; ${ }^{\ddagger \neq \neq \ddagger D}$ Department of Hematology, National Cancer Center Hospital, Tokyo, Japan; ${ }^{\S \S \$}$ Department of Hematology, Academic Medical Center, Amsterdam, Netherlands; IIIIIIIIUniversity of Michigan, Ann Arbor, MI, U.S.A.; \#\#\#\#ohns Hopkins School of Medicine, Baltimore, MD, U.S.A.; *****Department of Medicine and Biosystemic Science, Kyushu University Hospital, Fukuoka, Japan; ${ }^{\dagger+\dagger \dagger}$ Fondazione IRCCS Istituto Nazionale dei Tumouri, University of Milan, Milan, Italy; ${ }^{\ddagger \neq \neq \neq \neq \neq \ddagger \ddagger \neq N o v a r t i s ~ P h a r m a c e u t i c a l s ~}$ Corporation, East Hanover, NJ, U.S.A.; ${ }^{\S \S \S \S \S}$ Novartis Healthcare Pvt. Ltd., Hyderabad, India; $\|||||||||$ Novartis Institutes for Biomedical Research, Cambridge,

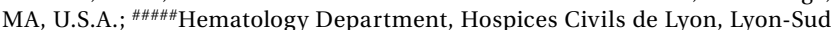
Hospital, Pierre-Bénite, France.

\section{Increasing capacity for autologous stem-cell transplants:} a quality improvement study

Uday Deotare, ${ }^{* \neq \ddagger}$ Adrienne Fulford, ${ }^{* \neq}$ Anargyros Xenocostas, ${ }^{*+\ddagger}$

Susan Nugent, ${ }^{* \neq}$ Susan Reiger,* Mark Mussio, ${ }^{*}$ Deanna Caldwell, ${ }^{* \neq}$ Kim Fletcher, ${ }^{*}$ Cadance Halley, ${ }^{*}$ Christine Soong, ${ }^{\S}$ Alan Gob*t

Introduction Autologous stem cell transplant (ASCT) is a standard of care for patients with multiple myeloma (MM) and Hodgkin and non-Hodgkin lymphoma (NHL). Traditionally these patients are admitted over a period of 21-28 days for this process. We tried to address the issue of increasing capacity for ASCT at London Health Sciences Centre (LHSC) by studying the root causes and carrying out this project as a quality improvement study. Hypothesis and Rationale In the last 3 years there has been an increased number of eligible patients for ASCT but with the same number of available inpatients beds. Hence, to increase the capacity for ASCT, it was decided to follow the Model for Improvement principle. Once major root causes were identified, a select group of interventions that focus on the underlying causes were targeted for improvement. One of the major root causes was increased length of stay of ASCT patients. It was planned to refine decreasing the length of stay of ASCT patients by converting inpatient chemotherapy to outpatient chemotherapy and by preventing readmissions. This intervention would be based on a continuous process of developing and conducting small tests of change [Plan-Do-Study-Act (PDSA) cycles].

The admission census for hematology for the last 3 years was obtained from Decision Support at LHSC. This pointed out that the majority of hematology patients' stay was related to ASCT. Audit was carried out on inpatient charts for both hematology and ASCT patients. The root causes leading to increased stay (LOS) was plotted on a Pareto chart [see Appendix I (https:// www.cttcanada.org/resource/resmgr/2020 conference/abstracts/27.png)]. Objective Our main objective or Big Dot aim was to increase the capacity of ASCT at LHSC by $20 \%$ by December 2020 [see Driver diagram, Appendix II ( https://www.cttcanada.org/resource/resmgr/2020_conference/ abstracts/28.png)]. The project aim was to decrease the LOS in ASCT by 2 days by May 2020 .

Methods The following change ideas were developed: change of inpatient (IP) conditioning chemotherapy to outpatient (OP) and reducing readmissions for post-transplant complications. This was undertaken initially for NHL (BEAM) and then would be extended to MM (melphalan). The project idea was conceived in September 2019 and PDSAs devised and tested in December 2019. The first PDSA ramps consisted of conversion of IP PowerPlan in the Cerner system (Cerner Corporation, Kansas City, MO, U.S.A.) to OP PowerPlan for administration of op chemotherapy. This was aligned with the creation of Flex Beds (IP beds which can be flipped as OP beds and vice-versa). The second PDSA ramps consisted of creation of a Costars (Stacey D, Carley M. Can Oncol Nurs J2017;27:92-8) tool which was modified for transplant conditioning chemotherapy. This assessment tool was used by incharge nurses to determine the risk pathway for readmissions. The baseline data of Los from November 2018 to October 2019 were plotted on a Shewart process control chart in an "I Chart" using QI Macros software, as shown in Appendix III (https://www.cttcanada.org/resource/ resmgr/2020_conference/abstracts/29.png).

Results The median Los was 24 days. After the implementation of the change idea with the first patient on 10 December 2019, the LOS of this ASCT patient decreased from median of 24 days to 16 days. The project is ongoing and will be updated at the time of the CTTC annual meeting.

Conclusions Increasing in the capacity for ASCT by decreasing the LOS is possible and feasible in a stepwise manner. We tried to address this root cause by change of IP conditioning chemotherapy to an OP setting and by 
preventing readmissions as a balancing measure. At the time of abstract submission only 1 patient has been trialed on this pathway and a full outcome of this study is to be presented in detail at the CTTC conference.

Affiliations: *London Health Sciences Centre, London, ON; ’Division of Hematology, Department of Medicine, Schulich School of Medicine and Dentistry, University of Western Ontario, London, ON; ${ }^{\ddagger}$ Blood and Marrow Transplant Program and ${ }^{\S}$ University of Toronto, Sinai Health, Toronto, ON.

\section{Outcome of hematopoietic stem-cell transplant for severe aplastic anemia in Pakistan}

Uzma Zaidi, Shafaq Samad, Maria Zahid, Nayab Soomro, Tahir S. Shamsi

Background In Pakistan, approximately 400-500 new cases of aplastic anemia are diagnosed every year and variant environmental exposures are accredited as an etiological factor. Only $20 \%-25 \%$ of patients receive stem cell transplants due to limited health resources.

Purpose To determine the overall survival, risk factors for relapse, and non-relapse mortality after matched-sibling donor transplant for severe aplastic anemia.

Methods It was a non-interventional prospective study, conducted at National Institute of Blood Disease and Bone Marrow Transplantation from 2011 to 2017. Severe aplastic anemia was diagnosed according to Camitta classification. Conditioning regimens used were fludarabine/ cyclophosphamide or fludarabine/anti-thymocyte globulin (ATG). The patients were closely followed for engraftment, primary or secondary graft failure, acute and chronic graft-versus-host disease (GvHD), bacterial viral and fungal infections during post-transplant phase.

Results One hundred and four patients underwent hematopoietic stem cell transplantation. Median age of patients was 12.5 years (3-45 years). The stem-cell source was peripheral blood in $55(61 \%)$, bone marrow in 37 (39\%), and both in $13(12.5 \%)$ patients. Mean stem-cell dose was $4.8 \pm$ 2.9 cells $/ \mathrm{kg}$ recipient weight. Overall survival at 6 years was $63 \%$. Primary and secondary graft failure occurred in $15 \%$ and $16 \%$ of patients respectively. Acute GvHD of grade I-II and grade III-IV GVHD occurred in 4/104 (3.8\%) and $2 / 104(1.9 \%)$ patients respectively. Chronic GvHD was observed in 6/92 $(6.1 \%)$ of patients. Fungal and bacterial infections were observed in $6.1 \%$ and $15.5 \%$ respectively. Cytomegalovirus (CMV) reactivation occurred in $11 \%$ patients, but CMV disease (CMV pneumonitis) was observed in 1 patient only. BK virus-associated hemorrhagic cystitis was observed in 2/104 (1.9\%) patients, managed with conservative treatment.

Conclusions Overall survival and quality of life of aplastic anemia patients has dramatically improved with the advent of stem-cell transplant in Pakistan. The transplant program needs further expansion in order to cater to the needs of patients coming from different regions of the country.

Affiliation: National Institute of Blood Disease and Bone Marrow Transplantation, Karachi, Pakistan.

52. Hematopoietic recovery and transfusion need after haploidentical transplantation in beta thalassemia patients_data from a developing country

Uzma Zaidi, Tahir S. Shamsi, Saqib Hussain Ansari, Tasneem Farzana

Background At present, thalassemia-free survival after matched-related and unrelated donor transplant is about $80 \%-90 \%$. Despite of bone marrow donor registries and cord blood banks, $20 \%-25 \%$ of the patients still do not find a suitable matched donor. Haploidentical stem-cell transplant (HISCT) is an alternative transplant option for these patients. We aimed to determine the outcome of HISCT in patients with thalassemia.

Purpose The aim was to establish this modality of treatment as standard of care for those who do not have a matched sibling donor.

Methods Between 2014 and 2018, 16 patients with thalassemia underwent HISCT. The median age of patients was 5 (1-8) years with a preponderance of men $(n=10,62.5 \%)$. Stem-cell source was bone marrow in $5(31 \%)$ and peripheral blood in $11(69 \%)$ patients. Mean stem-cell dose was $5.6 \pm 2.9 \times 10^{6}$ cells $/ \mathrm{kg}$. Standard preparative regimen and graft-versus-host disease (GvHD) prophylaxis was given. Patients were observed for hematopoietic recovery and transplant-related mortality including acute and chronic GvHD, primary and secondary graft failure, and infectious complications. Results Nine $(56.25 \%)$ of 16 patients were engrafted with full donor chimerism. Twelve (75\%) patients belonged to Pesaro class I, and 4 (25\%) to class II patients. Median time to neutrophil and platelet engraftment were 13 (11-20) and16 (12-36) days respectively. Primary and secondary graft failure was observed in $3(19 \%)$ and 4 (25\%) patients respectively Acute GVHD of gut and skin (grade II-III) was observed in 2 patients each within the first 100 days post-transplant. Cytomegalovirus reactivation occurred in $50 \%$ of patients. Invasive fungal infection was not observed in any of the patients. Culture-proven bacterial infection was documented in $62 \%$ of patients requiring intravenous antibiotics. Overall survival and relapse-free survival, were $81.25 \%$ and $56.25 \%$ over a median follow-up of 500 (21-1757) days.

Conclusions In view of our results, haploidentical transplant is a suitable modality for patients with thalassemia lacking a full-matched donor in Pakistan.

Affiliation: National Institute of Blood Disease and Bone Marrow Transplantation, Karachi, Pakistan.

53. Use of plerixafor in older patients undergoing autologous stem-cell transplant: a single institutional experience

Anna MacDonald, Ronan Foley, Josie Hallas

Background Autologous stem cell transplant (ASCT) is an effective therapeutic strategy for patients with a variety of hematologic malignancies. Expanding indications, utilization in older adults ( $>65$ years of age), and the availability of novel non-chemotherapy-based mobilization strategies have increased the number of transplants performed in most institutions. Plerixafor (Mozobil: Sanofi Genzyme, Cambridge, MA, U.S.A.) is a molecule that enhances the mobilization of autologous progenitor cells through binding of the chemokine receptor CXCR4. Plerixafor is currently funded to include patients who fail to collect at least $50 \%$ of target CD34+dose on day 1 or if the pre-absolute CD34+ level is $<10 / \mu \mathrm{L}$.

Purpose To investigate retrospectively collection variables on the final CD34+ count in older patients with non-Hodgkin lymphoma (NHL) and multiple myeloma (MM).

Methods A retrospective analysis was conducted evaluating a cohort of 168 consecutive patients ( $67 \mathrm{NHL}, 101 \mathrm{MM}$ ) undergoing autologous stem-cell transplant during the period from 1 January 2019 to 15 December 2019. The 2019 HPC and apheresis collection results from harvest MM and NHL patients were reviewed. The variables studied included age of patient, disease type, CD34 target, number of collection days required to meet CD34 target, use of cyclophosphamide, need for plerixafor, and patient recollection.

Results The median patient age was 61 (range: 18-76) and median number of days of collection was 2.4. Chemo mobilization (cyclophosphamide $2.5 \mathrm{~g} / \mathrm{m}^{2}$ ) was used in $42 \%$ of patients, and $56 \%$ of all patients required plerixafor. Repeat collections were required in $9 \%$ of patients. Mobilization failures occurred in only $2(<1 \%)$ patients. Plerixafor was used in NHL $(57 \%)$ and $57 \mathrm{MM}(56 \%)$ patients. In patients $\geq 65$, plerixafor was required in $39 \%$ of NHL and $33 \%$ of MM patients compared with $60 \%$ and $66 \%$ in patients $<65$ years of age.

Efficacy of plerixafor was assessed by a simple fold-increase ratio of day 2 absolute CD34+/ $\mu \mathrm{L} /$ day 1 absolute CD34+/ $\mu \mathrm{L}$. The mean fold increased for all NHL was 6.0 and all MM was 4.4 . In patients $\geq 65$, the mean fold increase was 7.4 in NHL and 3.9 in MM. In patients $<65$ years of age, the mean fold increase was 7.7 in NHL and 6.8 in MM. We found a median nextday fold increase of 4 in all patients. Plerixafor was safe with no serious adverse events reported in all $(n=95)$ patients.

Conclusions Plerixafor appears to be safe and well tolerated in older patients. Increases in CD34 levels were similar in older and younger patients. In our study, plerixafor was used more frequently in patients $<65$. Fold increase was higher in NHL patient's $\geq 65$ and $<65$ compared with MM patients. Mobilization failure was $1 \%$ even with the use of plerixafor.

Affiliation: Stem Cell Laboratory, Juravinski Hospital, Hamilton Health Sciences, Hamilton, ON.

\section{Does TNC impact engraftment in pediatric bone marrow transplant?}

Divya Subburaj, Kini Sandhu, Jacob J. Rozmus, Amanda M. Li

Introduction Bone marrow remains the preferred graft source in hematopoietic stem-cell transplants (HSCT) in children. Human leucocyte antigen (HLA)-match related donors (MRD) are preferred over unrelated donors (MUD). In pediatric HSCT, MRDs are commonly pediatric siblings while MUDs are adults, and therefore MRDs are more likely to be restricted by maximum harvest volume limits. The impact of cell dose on engraftment and clinical outcomes in pediatric НSCT is unclear, although target minimum total nucleated cells (TNC) of $3 \times 10^{8} / \mathrm{kg}$ has been extrapolated from the adult literature. Here, we describe the cell dose of all matched bone marrow allografts from a single pediatric institution and the associated clinical outcomes.

Methods A retrospective chart review was performed to survey fully matched allogeneic bone marrow derived HSCTs performed at BC Children's Hospital between 2008 and 2017.

Results A total of 69 HLA-matched donor HSCTs were identified. Two patients were excluded due to incomplete data. Median recipient age was 10 years (range: $1-17$ ). There were 31 MRDs and 36 MUDs. Hematologic 
malignancy ( $n=44$ ) was the most common indication for transplant. Median TNC was $2.72 \times 10^{8} / \mathrm{kg}$ (range: $1.06-11.7$ ) for MRD and 3.25 (range: $0.47-8.25$ ) for MUD grafts ( $p=0.3$ ). The median CD34 count was $4.54 \times 10^{6}$ / $\mathrm{kg}(1.27-13.57)$ for MRD and $3.56(1.2-8.1)$ for MUD grafts $(p=0.09)$. Median neutrophil engraftment was shorter with MRD at 18 days (range: 11-33) compared with MUD at 25 days (range: 11-45), though not statistically significant $(p=0.08)$. Those who developed bacteremia in the first month of HSCT had a lower mean CD34 count than those who did not $\left[3.67 \times 10^{6} / \mathrm{kg}\right.$ (SD $1.23, n=19$ ) vs. $4.74 \times 10^{6} / \mathrm{kg}$ (SD $\left.2.74, n=48\right) ; p=0.03$ ].

Thirty-two of the 67 patients $(48 \%)$ had grafts with low TNC (that is, $<3 \times 10^{8} / \mathrm{kg}$ ). Nineteen were MRDs with median TNC of $2.01 \times 10^{8} / \mathrm{kg}$ (range: $1.06-2.85)$ and median CD34 count of $4.28 \times 10^{6} / \mathrm{kg}(1.27-9.14)$. All 19 MRD recipients engrafted at a median neutrophil engraftment time of 21 days (range: 11-33). Thirteen patients with low TNC were MUDs, with a median TNC of $1.76 \times 10^{8} \mathrm{~kg}(0.47-2.93)$ and median CD34 of $3.56 \times 10^{6} / \mathrm{kg}(1.2-8.1)$. Eleven of the 13 MUDs engrafted, with a median time of 26 days (11-45). Two MUD recipients died of infection prior to engraftment; one at day +8 (gramnegative sepsis), and one at day +26 [Epstein-Barr virus (EBV) viremia] . Conclusions We observed that despite low TNC dose, neutrophil engraftment occurred in all matched related and most unrelated donor transplants over an acceptable timeframe. Two patients died of early infection prior to engraftment, and those who developed bacteremia in the peri-engraftment period had a lower mean CD34 count. There was a trend towards quicker neutrophil engraftment with MRDs compared with MUDs, even in the low TNC group, and no patients in this cohort required graft rescue for primary graft failure. Therefore, TNC in pediatric matched bone marrow transplants may not impact engraftment.

Affiliation: BC Children's Hospital, Vancouver, BC.

\section{CD56bright natural killer regulatory cells phenotyping} characterization in filgrastim-stimulated donor stem cells and association with graft-versus-host disease after hematopoietic stem-cell transplantation

Shima Azadpour,* Amina Kariminia,* Sayeh Abdossamadi,* Elena Ostroumov, ${ }^{*}$ Kirk R. Schultz*†

Background Previously, the CBMTG0601 trial demonstrated a higher chronic graft-versus-host disease (cGvHD) frequency associated with a lower proportion of CD56bright natural killer (NK) regulatory cells in the donor product. In fact, it appeared that the mechanism for the higher rate of cGvHD with a peripheral blood donor product was primarily associated with lower CD56bright NK regulatory cells in the filgrastim-stimulated apheresis peripheral blood product. The importance of CD56bright NK cells association with the absence of cGvHD has been subsequently observed associated with other plasma markers such as CXCL10 and responses to extracorporeal photopheresis. Little is still known about the function of the CD56bright NK-reg population. The current study focuses on further characterization of the phenotype of the CD56bright NK population in a small set of archived fligrastim-stimulated bone marrow or mobilized stem-cell biospecimen from the CBMTG0601 study.

Methods Participants were divided into groups based on the recipients' GvHD outcome. Three groups were included in the study: tolerant, with an absence of both acute and chronic GvHD $(n=8)$; de novo cGvHD with no previous acute GVHD $(n=9)$; those with both acute GvHD and chronic GVHD $(n=19)$.We used multi-parameter flow cytometry technique to characterize CD56bright and CD56dim NK cell subsets in fligrastim-stimulated bone marrow or mobilized peripheral blood stem cells. CD56bright NK cells were defined by high expression of CD56 and were CD16-. CD56dim NK cells had low CD56 expression and were CD16+. Data were acquired using LSR II flow cytometer (BD Biosciences) and analyzed by FlowJo v10.0. In all graphs, the mean and SD were calculated and plotted. SPSS v.22 software was used, and $p$ values $(*<0.05)$ were considered significant.

Results The expression level of different markers in 2 subsets CD56bright and CD56dim NK populations: CD94, CXCR3, CD119, CD69, CD158 were significantly higher on CD56bright NK cells compared with the CD56dim NK population. We analyzed for qualitative differences in the CD56bright NK population that associated with patients who developed both no acute GVHD (aGvHD) and cGvHD (tolerant) compared with those who developed either de novo cGvHD or had cGvHD with previous aGvHD (non-tolerant). CD56 bright NK cells in patients were tolerant compared with the non-tolerant group had significantly higher expression of CD94 (50.0 effect ratio [ER]), CD27 (10.0 ER), and CD158 (2.5 ER) and significantly lower expression of CD314 (0.12 ER).

Conclusions Donor stem-cell CD56bright NK cells over-express additional cell surface markers compared with CD56dim NK cells, potentially helping in identifying and isolating this population. Moreover, a subpopulation of
CD56bright NK cells is associated with immune tolerance in patients that do not develop both aGvHD and cGvHD and have a unique phenotypic signature. Confirmation on larger patient populations is needed.

Affiliations: *Michael Cuccione Childhood Cancer Research Program, BC Children's Hospital, Department of Pediatrics, University of British Columbia, and ${ }^{\dagger}$ Division of Pediatric Hematology/Oncology/Blood and Marrow Transplant, BC Children's Hospital, Vancouver, BC.

\section{Allogeneic hematopoietic stem-cell transplantation (allo-HSCT)} in a patient with Prader-Willi Syndrome-unique considerations Tristan E. Knight, * Jane Lowry, ${ }^{*}$ Sarah Leppington, ${ }^{+}$Donna A. Wall,* Jennifer Seelisch ${ }^{\dagger}$

Introduction Prader-Willi syndrome (PWS) is a genetic condition arising from mutations of paternal chromosome $15 \mathrm{q}$, or maternal uniparental disomy of chromosome 15 . We report a patient with pws who developed myelodysplastic syndrome (MDS) necessitating allo-HSCT, highlighting unique consideration of his transplant course.

Clinical Course A13-year-old boy with Pws developed pancytopenia with circulating blasts; bone marrow evaluation showed MDS; PWS-associated comorbidities included treatment with recombinant human GH (rhGH) for $10 \mathrm{yrs}$ (age 3y-diagnosis), obesity (99th centile), anxiety/compulsive behaviours, developmental delay, and severe obstructive sleep apnea (OSA). He received 2 courses of azacytidine followed by allo-HSCT 3 months after diagnosis using cell 10/10 HLA matched unrelated donor and mobilized peripheral blood hematopoietic stem cells. Preparative regimen was daily busulfan $(3.2 \mathrm{mg} / \mathrm{kg}$ actual weight initial dose with pharmacokineticguided adjustment to target an AUC of $4478 \mu \mathrm{M}^{*}$ min over 4 days) plus cyclophosphamide $120 \mathrm{mg} / \mathrm{kg}$ based on adjusted ideal body weight and antithymocyte globulin (ATG) $4.5 \mathrm{mg} / \mathrm{kg}$ actual weight. Graft-versus-host disease (GvHD) prophylaxis was tacrolimus/methotrexate. A comprehensive team approach was implemented, with nursing, child life, physical therapy, and medical team involvement, including distraction/redirection techniques, avoidance of mood-altering medications, pill-taking strategies, calming room environment, and strong family support. A small, familiar outpatient care team was used following discharge-phlebotomist, nurse, and physician-to minimize anxiety. There were no unexpected transplant-related complications: grade 2 oral mucositis, grade 2 hypertension, Staphylococcus aureus bacteremia with early removal of central line, and Epstein-Barr virus (EBV) reactivation treated with rituximab. He has exhibited no GvHD. Full donor engraftment occurred on day +17 , and he was discharged to outpatient care on day +20 . He was able to maintain oral intake throughout, likely due to the pathologic lack of satiety characteristic of Pws. Discharge weight was $1.4 \mathrm{~kg}$ below admission weight. During the transplant, his anxiety/ compulsion behaviours worsened but were manageable. Following discharge, weight gain became pronounced, peaking at $92.5 \mathrm{~kg}$ (99.8th centile; $13.3 \mathrm{~kg}$ over 2 months). Return to his previous routines and home environment allowed for stabilization of his weight gain, OCD, and anxiety.

Conclusions Literature review prior to transplant failed to identify evidence of DNA repair defects or other vulnerabilities which would require modification of the preparative regimen. With an intensive multidisciplinary approach, our patient tolerated the allo-HSCT process. The loss of familiar home structure and associated controls likely contributed to his rapid post-transplant weight gain and worsening of psychiatric comorbidities. Based upon our experience, we see no reason to exclude patients with PWS from allo-HSCTs due to PWS diagnosis itself.

Affiliations: *The Hospital for Sick Children and University of Toronto, Toronto, $\mathrm{ON} ;{ }^{\dagger}$ Children’s Hospital, London Health Sciences Centre, and Western University, London, ON.

\section{Early warning of infection in patients undergoing} hematopoietic stem cell transplantation using heart rate variability and serum biomarkers

C. Arianne Buchan, ${ }^{*}$ Heidi Oi-Yee Li, ${ }^{\dagger}$ Christophe L. Herry, ${ }^{\ddagger}$ Christopher Bredeson, $\$$ Paul MacPherson,* Elliott Faller,* Michael Hodgins, ${ }^{\S}$ Nathan B. Scales, ${ }^{\ddagger}$ Andrew J.E. Seelyll

Background Neutropenia commonly occurs in patients undergoing hematopoietic stem-cell transplantation (HSCT), leading to increased risk of infection and mortality. Early identification of infection is critical to prevent clinical deterioration; however, current techniques to diagnose infection are imprecise and uncertain, which may lead to delayed diagnosis (with risk of profound deterioration) or overtreatment (with risk of microbial resistance). Given that heart rate variability (HRV) analysis is a sensitive early marker for infection and serum inflammatory biomarkers have been shown to be specific for infection, we hypothesized their combination may be useful for early warning of infection. 
Purpose The objective was to develop and evaluate a composite model utilizing HRV with daily serum biomarker measurements to provide daily risk stratification of subsequent deterioration in HSCT patients within the following 48 hours.

Methods Consent was obtained from 116 ambulatory outpatients prior to undergoing НSСт between 2007 and 2017 to undergo observational electrocardiogram (ECG) continuous monitoring 24h pre-transplant untilinfection or 14 max days post-transplant. Patients had daily visits, vital signs, and blood serum sampling frozen $\left(-80^{\circ} \mathrm{C}\right)$ for subsequent biomarker analysis. Demographic and clinical variables, and outcomes were collected. Infection onset was defined as the time of broadening of antibiotics. Indications for antibiotic broadening were adjudicated as "true infection" or not by 2 blinded HSCT clinicians. For each patient, HRV analysis was performed (8 HRV metrics, 10-min windows) and composite variability time series created. The probability of deterioration within the subsequent $48 \mathrm{~h}$ was estimated using a logistic regression model trained on the composite HRV, and further combined with serum biomarkers features (TNF $\alpha$, IL-6 and IL-7) if the HRV-based probability exceeded a median threshold, using a rule-based Naïve Bayes model.

Results Of 116 patients enrolled, 35 (30\%) withdrew with less than $24 \mathrm{~h}$ of useable HRV data resulting in a cohort of 81 included patients of whom 35 (43\%) had serum biomarker data, 48 (59\%) had antibiotic broadening adjudicated as true infection [Figure 1 Probability of deterioration in patients with documented infection (red) vs. those without documented infection (green) leading up to the time of diagnosis of infection (https:// www.cttcanada.org/resource/resmgr/2020_conference/abstracts/30. png)]. The number of patients at each time point varied depending on availability of data and duration of participation in the study. The combined HRV-biomarker predictive model started increasing $~ 48$ hours on average prior to diagnosis of infection, could distinguish between high risk ( $>90 \%$ incidence of subsequent infection within $72 \mathrm{~h})$, average risk $(\sim 50 \%)$ and low risk $(<10 \%)$, and achieved an area under the receiver operating characteristic curve (AUC-ROC) of 0.87 [Figure 2 Fold increase in risk of deterioration within the next 72 hours, corresponding to the daily assessment of probability derived from the combined HRV and biomarker-based model. Total number of days across patients: 69 (https://www.cttcanada.org/resource/ resmgr/2020_conference/abstracts/31.png)].

Conclusions For the first time, we derived a combined predictive model using HRV-based probability of deterioration within $24 \mathrm{~h}$ combined with daily biomarker inflammatory markers which may assist with early warning of infection. However, prophylactic predictive ECG monitoring and daily serum collection proved intolerable or impossible for many patients. These results require multicentre validation.

Affiliations: *Division of Infectious Diseases, Department of Medicine, The Ottawa Hospital, ${ }^{\dagger}$ Faculty of Medicine, The Ottawa Hospital, University of Ottawa, ${ }^{\ddagger}$ Clinical Epidemiology Program, The Ottawa Hospital Research Institute, ${ }^{\S}$ Division of Hematology, Department of Medicine, The Ottawa Hospital, and $\|$ Division of Critical Care Medicine, Department of Medicine, The Ottawa Hospital, Ottawa, ON.

\section{Characteristics of hepatic veno-occlusive disease from the Cell Therapy Transplant Canada registry: a prospective observational study}

Kevin Brown, ${ }^{* \dagger}$ Kristjan Paulson, ${ }^{* \dagger}$ Véronique Baribeau, ${ }^{\ddagger}$ Jean Lachaine ${ }^{\ddagger \S}$

Background Hepatic veno-occlusive disease (VOD) is a major complication of hematopoietic stem cell transplantation (HSCT). There is limited Canadian evidence on the incidence of vOD, risk factors for development of VOD, and VOD treatment patterns.

Purpose The objective of this study was to describe Canadian patients who underwent HSCT and subsequently developed vOD.

Methods This retrospective observational study used data from the Cell Therapy Transplant Canada (CTTC) registry, a database containing detailed clinical information on consecutive patients undergoing HSCT in most Canadian centres. Data were collected at the time of HSCT and at regular intervals (day 100, 6 months, 18 months, 24 months, and then annually) following HSCT. From 2013 to 2019, patients with VOD were identified from the medical charts and compared with those who did not develop voD. Both non-severe and severe voD cases were eligible for inclusion. Only the first reported НSCT per patient was included in this analysis. Children were defined as being $<18$ years of age on the date of transplant. Some data were missing due to the nature of a registry database. Results were calculated using the overall number of patients in each group.

Results From the registry, 64 vOD patients and 2339 non-vOD patients who had undergone HSCT were identified. The proportion of patients with VOD after HSCT was $2.7 \%$. Most patients were undergoing their first НSCT: $84.4 \%$ in VOD patients and $89.8 \%$ in non-VOD patients. The most common underlying diagnosis in both groups was acute myeloid leukemia $(31.3 \%$ and
$38.5 \%$ respectively). Of the 64 voD patients, 27 were children (42.2\%), 24 were adults $(37.5 \%)$, and 13 had missing age data $(20.3 \%)$. Correspondingly, in the 2339 non-VOD patients, 396 were children (16.9\%), 1943 were adults (83.1\%), and none had missing data. The median age of adult patients was slightly higher for those with VOD (56.7 years; IQR: 7.9) than those without VOD (54.1 years; IQR: 19.2). The most frequently used conditioning regimen in both groups was high-dose busulfan $(42.2 \%$ in voD patients; $47.7 \%$ in non-VOD patients). Prophylaxis for liver toxicity (for example, defibrotide, $\mathrm{N}$-acetylcysteine, tissue plasminogen activator, ursodiol) was recorded for 55 VOD patients and 1192 non-VOD patients and was more frequently used post-HSCT in patients who developed VOD $(71.9 \%)$ than in patients who did not $(32.0 \%)$. Ursodiol was the most common prophylaxis in each group ( $64.1 \%$ and $30.2 \%$ respectively). Resolution of VOD was observed in $71.9 \%$ of affected patients.

Conclusions Compared with non-vOD patients, a higher percent of VOD patients were children, and adult patients with voD were typically older. High-dose busulfan as a conditioning regimen and ursodiol as a liver toxicity prophylaxis were frequently used in both VOD and non-voD patients. More in-depth analyses will be performed to analyze the incidence of VOD by years, as well as the risk factors related to voD.

Affiliations: *CancerCare Manitoba and ${ }^{+}$University of Manitoba, Winnipeg, MB; ${ }^{\ddagger}$ PeriPharm and ${ }^{\S}$ University of Montreal, Montreal, QC.

\section{Outcomes following cellular therapy for relapsed/refractory Hodgkin lymphoma in the era of novel therapy}

Olivier Veilleux, Habiba Alaoui, Jean-Sébastien Claveau, Imran Ahmad, Nadia M. Bambace, Léa Bernard, Sandra Cohen, Jean-Sébastien Delisle, Thomas Kiss, Jean Roy, Denis-Claude Roy, Guy Sauvageau, Silvy Lachance

Background Up to $30 \%$ of patients with advanced stage classical Hodgkin lymphoma (cHL) will be primary refractory or experience disease recurrence following front-line therapy. High-dose chemotherapy followed by autologous hematopoietic stem-cell transplantation (autoHSCT) remains the standard of care for relapsed refractory disease. Unfortunately, about half of patients who benefit from autoHSCT will experience recurrence. New molecules have recently been approved for the treatment of relapsed/ refractory $(\mathrm{R} / \mathrm{R})$ Hodgkin lymphoma questioning the role and sequence of HSCT in cHL treatment. To better define the role of cellular therapy in cHL management, this study aims to review the long-term outcome and curative potential of both autologous and allogeneic HSCT for the treatment of R/R cHL in light of the results associated with novel therapies.

Methods This single-centre, research and ethics committee-approved retrospective study included 93 consecutive patients referred to Hôpital Maisonneuve-Rosemont, between January 2007 and December 2019, for R/R cHL and treated with second-line autologous HSCT. Patients not achieving CR prior to transplant or relapsing after autologous HSCT were candidates for tandem or salvage allogeneic HSCT. All patients were treated according to standard protocols. Clinical data were extracted from the HSCT program database.

Results Of the 93 consecutive R/R cHL patients included in the analysis and who received autoHSCT, 18 (19\%) also received alloHSCT, 4 of whom as tandem auto/allo approach. The 5 -year overall survival (OS) is $80 \%$ (95\% CI: $68 \%$ to $87 \%$ ), and median os is 13.1 years [Figure 1 (https://www.cttcanada. org/resource/resmgr/2020_conference/abstracts/32.png)]. The 5-years progression-free survival (PFS) for the autoHsct cohort, is 55\% (95\% CI: $43 \%$ to $65 \%$ ), with a median PFS of 6.8 years [95\% CI: 3.7 to NA; Figure 2 (https:// www.cttcanada.org/resource/resmgr/2020_conference/abstracts/33. png)]. Relapse following autoHSCT occurred in 24 patients (25.8\%) with a median time to relapse of 9 months. Of these, 18 patients received alloHSCT, 5 from a matched related, 9 from a matched unrelated donor and 4 received cord blood. All patients achieved a CR at day +100 . The 5 -years os is $89 \%(95 \%$ CI: $62 \%$ to $97 \%$ ); the median os is still not reached [Figure 3 (https://www. cttcanada.org/resource/resmgr/2020_conference/abstracts/34.png)]. The 5- years PFs following alloHSCT is $78 \%$ (95\% CI: $51 \%$ to $91 \%$ ), and median PFS is still not reached [Figure 4 (https://www.cttcanada.org/resource/ resmgr/2020_conference/abstracts/35.png)]. Acute GvHD was diagnosed in $7 / 18$ patients, one of which was severe and of grade III (5.6\%). Moderate to severe chronic GvHD developed in 5/18 patients (27.8\%).

Conclusions These results demonstrate that autologous HSCT allow robust long-term PFS while alloHSCT provide excellent long-term results after autoHSCT relapse. In comparison, the ORR and CR rate following PD-1 inhibitor treatment for R/R cHL is $67 \%$ and $20 \%$, with a median duration of response of 11.1 months. Although the number of patients treated with alloHSCT in our study is small, these results demonstrate that long-term DFS and os can be achieved with alloHSCT with acceptable toxicity for R/R disease. For treatment of R/R cHL, alloHSCT remains the only curative modality and delaying it may jeopardize long-term outcome. 
Affiliations: Hôpital Maisonneuve-Rosemont, Division of Hematology, Medical Oncology and Hematopoietic Cell Transplant Program, Université de Montréal, Montréal, Québec, QC.

\section{Safety and efficacy of tisagenlecleucel (CTL019) in children,} adolescents, and young adults: the Canadian trial and real-world experience

Joerg Krueger, ${ }^{* \dagger}$ Pierre Teira, ${ }^{\ddagger}$ Sarah Alexander, ${ }^{* \dagger}$ Amalia Souza, ${ }^{\ddagger}$ Stephanie Mourad, ${ }^{*+}$ Sumit Gupta, ${ }^{* \dagger}$ Johanne Richer, ${ }^{\ddagger}$ Tal Schechter, ${ }^{* \dagger}$ Muhammed Ali, ${ }^{*+}$ Kuang-Yueh Chiang, ${ }^{*+}$ Sonia Cellot, ${ }^{\ddagger}$ Johann Hitzler, ${ }^{*+}$ Angela Punnett, ${ }^{*+}$ Mylene Bassal, ${ }^{\S}$ David Mitchell,"l Bruno Michon, ${ }^{\sharp}$ Sandra Cohen, ${ }^{* *}$ Janie Charlebois, ${ }^{,+}$Beth Cairny, ${ }^{\neq \ddagger}$ Uma Athale, ${ }^{\$ \S}$ Michel Duval, ${ }^{\ddagger}$ Jim Whitlock, ${ }^{* \dagger}$ Henrique Bittencourt ${ }^{\ddagger}$

Background Outcomes for children, adolescents, and young adults with multiple relapsed and refractory $(\mathrm{R} / \mathrm{R})$ precursor $\mathrm{B}$ acute lymphoblastic leukemia (B-ALL) remains poor. Tisagenlecleucel (CTL019), a chimeric antigen receptor (CAR) T cell therapy targeting CD19 has been used in this high-risk population with promising results and achieved Health Canada approval in September 2018.

Purpose To describe the feasibility, safety, post-infusion management, and efficacy of tisagenlecleucel in Canadian children, adolescents, and young adults.

Methods Retrospective patient chart analysis of all patients who received tisagenlecleucel in Canada for R/RALLeither through clinical trials (NCT02435849, NCT03123939, $n=22)$ or as standard clinical care $(n=7)$ between 2015 and 2020. Kaplan-Meier curves were used for time-to-event analysis.

Results Thirty-three patients meeting treatment indications were referred to the two Canadian pediatric CAR T cell centres. Median age at initial diagnosis and at infusion were 6.7 years (IQR 9.5) and 9.8 years (IQR 7.8) respectively. Thirteen out of 33 (39\%) patients underwent prior allogeneic hematopoietic stem-cell transplantation, 5/33 (15\%) had Down syndrome. Apheresis was performed in 31/33 (94\%) patients, and in 2 (6\%) patients severe lymphopenia prevented collection. Though product was successfully manufactured for 29/31 (94\%) patients, 6/29 (21\%) patients required $>1$ manufacturing attempt and $4 / 29$ patients (14\%) were infused with an out-of-specifications product.

Twenty-five out of $29(86 \%)$ achieved molecular remission at 3 months. Estimated event-free survival and overall survival were, respectively, $70 \%$ (95\% CI: $53 \%$ to $91 \%$ ) and $82 \%$ (95\% CI: $68 \%$ to $98 \%$ ) at 6 months and $44 \%$ (95\% CI: $25 \%$ to $75 \%$ ) and $66 \%$ (95\% CI: $48 \%$ to $89 \%$ ) at 12 months with a median follow-up time of 221 days. Twelve (41\%) of patients relapsed (CD19 negative $n=5$, CD19 positive $n=7$ ). The latest relapse was seen 42 months from initial infusion. Two patients received a re-infusion, one for early B cell recovery, one for relapse.

Cytokine release syndrome (CRS) occurred in $22 / 29$ patients $(76 \%$, grade $3 / 4, n=3$ ), while neurotoxicityoccurred in $8 / 29$ patients $(28 \%$, grade $3 / 4$, $n=3)$. Six patients $(27 \%)$ received tocilizumab, $5 / 29$ patients $(17 \%)$ required ICU care, and no treatment-related deaths were observed.

Ten out of 25 responders ( $40 \%$ ) experienced neutropenia beyond 1 month, median time to neutrophil recovery $(>500$ cells $/ \mu \mathrm{L})$ was 79 days (IQR 82). One patient remains neutropenic at 9 months and one patient required a hematopoietic stem cell boost due to cytopenia/infectious complications. Thrombocytopenia $(<50000 \mathrm{PLT} / \mu \mathrm{L})$ beyond one month was observed in $11 / 25$ patients $(44 \%)$.

Conclusions Tisagenlecleucel is safe and effective in patients with $\mathrm{R} / \mathrm{R}$ B-ALL, though long-term outcomes are still unknown; CRS and neurotoxicity were mostly mild and managed without ICU-level support. Post-infusion care for prolonged cytopenia and monitoring of CAR $\mathrm{T}$ cell persistence requires continuous follow-up.

Affiliations: *Division of Hematology/Oncology/BмT, The Hospital for Sick Children, Toronto, ON; ${ }^{\dagger}$ Department of Pediatrics, University of Toronto, Toronto, ON ${ }^{\ddagger}$ Division of Hematology and Oncology, Cell Therapy Program, CHU Sainte-Justine, Montreal, QC; §Division of Hematology and Oncology, Children's Hospital of Eastern Ontario, Ottawa, ON; $\|$ Division of Hematology and Oncology, Montreal
Children's Hospital, Montreal, QC; "Department of Pediatrics, CHU de Quebec, Université de Laval, Quebec City, QC; **Division of Hematology and Oncology, Stem Cell Transplant Program, Maisonneuve-Rosemont Hospital, Montreal, QC; ${ }^{\dagger+}$ Division of Hematology and Oncology, CHu de Sherbrooke-Université de Sherbrooke, Sherbrooke, QC; ${ }^{\ddagger \ddagger}$ Division of Hematology and Oncology, Children’s Hospital, London Health Sciences Centre, London, ON; ${ }^{\S}$ Division of Hematology and Oncology, McMaster Children's Hospital, Hamilton Health Sciences, Hamilton, ON.

61. Addition of anti-thymocyte globulin to standard graftversus-host disease prophylaxis compared with standard treatment alone in patients with hematologic malignancies undergoing transplantation from unrelated donors: final analysis of a randomized, open-label, multicentre, phase 3 trial Irwin Walker, Tony Panzarella, Stephen Couban, Felix Couture, Gerald Devins, Mohamed Elemary, Geneviève Gallagher, Holly Kerr, John Kuruvilla, Stephanie J. Lee, John Moore, Thomas Nevill, Gizelle Popradi, Jean Roy, Kirk R. Schultz, David Szwajcer, Cynthia Toze, Ronan Foley

Background This is the final, 24-month follow-up of the CTTC 1901 trial Methods This was a randomized, open-label, multicentre, phase 3 trial. Patients between 16 and 70 years of age had a hematologic malignancy, a Karnofsky score of at least 60 and received an unrelated donor graft following myeloablative or non-myeloablative reduced-intensity conditioning. Patients received either anti-thymocyte globulin $4.5 \mathrm{mg} / \mathrm{kg}$ plus standard GVHD prophylaxis or standard GvHD prophylaxis alone. The primary endpoint was freedom from immunosuppressive therapy without resumption at 12 months. Here we report on the prespecified 24-month analysis.

Results A total of 203 eligible patients were recruited and randomly assigned to receive anti-thymocyte globulin $(n=101)$ or standard GVHD prophylaxis alone $(n=102)$. Of the 99 evaluable patients in the anti-thymocyte globulin group, 38 (38\%) were free from immunosuppressive therapy at 24 months compared with 18 (19\%) of 97 patients in the standard GvHD prophylaxis group [adjusted odds ratio (OR) 3.49 ; $95 \%$ CI: 1.60 to $7.60 ; p=$ 0.0016 ]. The cumulative incidence of relapse was $16.3 \%$ (95\% CI: $8.9 \%$ to $23.7 \%$ ) in the anti-thymocyte globulin group compared with $17.5 \%$ (95\% CI: $9.9 \%$ to $25.1 \%$ ) in the standard GvHD prophylaxis group ( $p=0.73$ ) and nonrelapse mortality was $21.2 \%$ (95\% CI: $13.2 \%$ to $29.2 \%$ ) compared with $31.3 \%$ (21.9\% to $40.7 \% ; p=0.15)$. The cumulative incidence of chronic GvHD was $26.3 \%$ (95\% СI: $17.5 \%$ to $35.1 \%$ ) in the anti-thymocyte globulin group and $41.3 \%$ (95\% CI: $31.3 \%$ to $51.3 \%$ ) in the standard GVHD prophylaxis group ( $p=$ 0.032 ). Overall survival at 24 months was $70.6 \%$ (95\% CI: $60.6 \%$ to $78.6 \%$ ) in the anti-thymocyte globulin group compared with $53.3 \%$ (95\% CI: $42.8 \%$ to $62.8 \%$ ) in the standard GvHD prophylaxis group [adjusted hazard ratio (HR): $0.56 ; 95 \%$ CI: 0.35 to $0.90 ; p=0.017$ ]. Symptoms of chronic GvHD by the Lee Scale were more prevalent in the standard GvHD prophylaxis group, with scores of 13.27 (SD 10.94) in the anti-thymocyte globulin group and 20.38 (SD 14.68) in the standard GVHD prophylaxis group $(p=0.040)$. Depressive symptoms were more prominent in the standard GvHD prophylaxis group, the mean Center for Epidemiological Studies Depression scale (CES-D) scores were 10.40 (SD 9.88) in the anti-thymocyte globulin group and 14.62 (SD 12.26) in the standard GvHD prophylaxis group $(p=0.034)$. Serious adverse events (CTCAE grade 4 or 5) occurred in $38(38 \%)$ patients in the antithymocyte globulin group and in $49(51 \%)$ in the standard GvHD prophylaxis group.

Interpretation The results of this prespecified 24-month analysis suggest that pretreatment with anti-thymocyte globulin provides clinically meaningful benefits when added to standard GvHD prophylaxis in patients undergoing unrelated donor transplantation, including decreases in use of immunosuppressive therapy, chronic GvHD and its symptoms, and depressive symptoms, and improved overall survival. Anti-thymocyte globulin should be included in the preparative regimens of patients with hematologic malignancies selected for unrelated donor transplantation.

Affiliation: Cell Therapy Transplant Canada, Vancouver, BC. 